ESAIM: PROCEEDINGS AND SURVEYS, September 2019, Vol. 66, p. 109-143

Philippe Helluy, Jean-Marc Hérard and Nicolas Seguin Editors

\title{
A HIERARCHY OF NON-EQUILIBRIUM TWO-PHASE FLOW MODELS
}

\author{
Gaute Linga ${ }^{1,2}$ And Tore FlÅtten ${ }^{3}$
}

\begin{abstract}
We review and extend a hierarchy of relaxation models for two-phase flow. The models are derived from the non-equilibrium Baer-Nunziato model, which is endowed with relaxation source terms to drive it towards equilibrium. The source terms cause transfer of volume, heat, mass and momentum due to differences between the phases in pressure, temperature, chemical potential and velocity, respectively. In the context of two-phase flow models, the subcharacteristic condition implies that the sound speed of an equilibrium system can never exceed that of the relaxation system. Here, previous work by Flåtten and Lund [Math. Models Methods Appl. Sci., 21 (12), 2011, 2379-2407] and Lund [SIAM J. Appl. Math. 72, 2012, 1713-1741] is extended to encompass two-fluid models, i.e. models with separately governed velocities for the two phases. Each remaining model in the hierarchy is derived, and analytical expressions for the sound speeds are presented. Given only physically fundamental assumptions, the subcharacteristic condition is shown to be satisfied in the entire hierarchy, either in a weak or in a strong sense.
\end{abstract}

Keywords Two-phase flow, relaxation systems, subcharacteristic condition Classification 2010 MCS. 76T10, 35L60

\section{INTRODUCTION}

The concurrent flow of two fluid phases occurs in a wide range of industrially relevant settings, including in nuclear reactors [11], petroleum production [1,9], heat exchangers [53], cavitating flows [58], and within carbon capture, transport and storage (CCS) [10,40,48]. However, for most simulation purposes, resolving the full three-dimensional flow field may be too cumbersome, due to the complex interaction between the phases. In particular, this encompasses calculating the temporal evolution of the interface between the phases, and the transfer of mass, heat and momentum across it. Averaging methods (see e.g. Ishii and Hibiki [34] or Drew and Passman [18]) may therefore be applied to avoid direct computation of the interface. The resulting coarse-grained models may often be expressed as hyperbolic relaxation systems with source terms accounting for the interactions between the phases, driving them asymptotically towards equilibrium at a finite rate. In a quasi-linear form, one-dimensional versions of such systems may be written as

$$
\partial_{t} \mathbf{U}+\mathbf{A}(\mathbf{U}) \partial_{x} \mathbf{U}=\frac{1}{\epsilon} \mathbf{Q}(\mathbf{U})
$$

\footnotetext{
1 PoreLab, The Njord Centre, University of Oslo, Norway. e-mail: gaute.linga@mn.uio.no

2 Niels Bohr Institute, University of Copenhagen, Copenhagen, Denmark.

${ }^{3}$ Dept. of Petroleum Engineering, University of Stavanger, Stavanger, Norway. e-mail: tore.h.flatten@uis.no
} 
wherein $\mathbf{U}(x, t) \in G \subseteq \mathbb{R}^{N}$ is the (smooth) vector of unknowns and $\mathbf{A}(\mathbf{U})$ is a matrix which we shall call the Jacobian of the system, in analogy to conservative systems. ${ }^{1}$ Further, $\epsilon$ is a characteristic time associated with the relaxation process described by $\mathbf{Q}(\mathbf{U})$. For an extensive review of the existing literature on such systems, see e.g. Natalini [50], or, for a more concise summary, consider the first few sections of Solem et al. [60] and the references therein.

Two limits of the relaxation system (1) will be considered in this paper:

- The non-stiff limit, corresponding to the limit $\epsilon \rightarrow \infty$. In this case, we may write (1) as

$$
\partial_{t} \mathbf{U}+\mathbf{A}(\mathbf{U}) \partial_{x} \mathbf{U}=0
$$

We will refer to (2) as the homogeneous system.

- The formal equilibrium limit, which is characterized by $\mathbf{Q}(\mathbf{U}) \equiv 0$. This defines an equilibrium manifold [12] through $\mathcal{M}=\{\mathbf{U} \in G: \mathbf{Q}(\mathbf{U})=0\}$. We now assume that the reduced vector of variables $\mathbf{u}(x, t) \in$ $\mathbb{R}^{n}$, where $n \leq N$, uniquely defines an equilibrium value $\mathbf{U}=\mathcal{E}(\mathbf{u}) \in \mathcal{M}$. We may then express (1) as

$$
\partial_{t} \mathbf{u}+\mathbf{B}(\mathbf{u}) \partial_{x} \mathbf{u}=0, \quad \mathbf{U}=\mathcal{E}(\mathbf{u}),
$$

where $\mathbf{B}(\mathbf{u})=\mathbf{P}(\mathbf{u}) \mathbf{A}(\mathcal{E}(\mathbf{u})) \partial_{\mathbf{u}} \mathcal{E}(\mathbf{u})$ is the Jacobian of the reduced system. Herein, the operator $\mathbf{P}(\mathbf{u})$ : $\mathbb{R}^{N} \rightarrow \mathbb{R}^{n}$ satisfies $\mathbf{P}(\mathbf{u}) \partial_{\mathbf{u}} \mathcal{E}(\mathbf{u})=\mathbf{I}_{n}$, i.e. the identity matrix. We will refer to (3) as the equilibrium system.

We expect solutions of (1) to approach solutions of (3) as $\epsilon \rightarrow 0$, i.e. in the stiff limit, where the relaxation towards equilibrium is assumed to be instantaneous.

\subsection{The subcharacteristic condition}

An essential concept which arises in the study of relaxation systems and their stability, is the so-called subcharacteristic condition. It was first introduced by Leray [38], subsequently independently found by Whitham [68], and later developed by Liu [41] for conservative $2 \times 2$ systems. For a general $N \times N$ relaxation system, such as (1), the condition may be formulated as follows.

Definition 1.1 (Subcharacteristic condition). Let the $N$ eigenvalues of the matrix $\mathbf{A}$ of the homogeneous system (2) be given by $\Lambda_{i}$, sorted in ascending order as

$$
\Lambda_{1} \leq \ldots \leq \Lambda_{i} \leq \Lambda_{i+1} \leq \ldots \leq \Lambda_{N}
$$

Similarly, let $\lambda_{j}$ be the $n$ eigenvalues of the matrix $\mathbf{B}$ of the equilibrium system (3). Herein, the homogeneous system (2) is applied to a local equilibrium state $\mathbf{U}=\mathcal{E}(\mathbf{u})$, such that $\Lambda_{i}=\Lambda_{i}(\mathcal{E}(\mathbf{u}))$, and $\lambda_{j}=\lambda_{j}(\mathbf{u})$. Now, the equilibrium system (3) is said to satisfy the subcharacteristic condition with respect to the homogeneous system (2) when (i) all $\lambda_{j}$ are real, and (ii) if the $\lambda_{j}$ are sorted in ascending order as

$$
\lambda_{1} \leq \ldots \leq \lambda_{j} \leq \lambda_{j+1} \leq \ldots \leq \lambda_{n},
$$

then the eigenvalues of the equilibrium system are interlaced with the eigenvalues of the homogeneous system, in the sense that $\lambda_{j} \in\left[\Lambda_{j}, \Lambda_{j+N-n}\right]$.

For general $N \times N$ conservative relaxation systems, Chen et al. [12] showed that if mathematical entropy is dissipated by the relaxation term, then the subcharacteristic condition follows, and the equilibrium system is endowed with a strictly convex entropy pair. Hence the subcharacteristic condition is necessary for this notion of entropy stability of the relaxation process. Note that the analysis of Chen et al. [12] cannot be straightforwardly

\footnotetext{
${ }^{1}$ In systems which can be written on the conservative form $\partial_{t} \mathbf{U}+\partial_{x} \mathbf{F}(\mathbf{U})=0$, we have that in the weak form $(2), \mathbf{A}=\partial_{\mathbf{U}} \mathbf{F}$ is the actual Jacobian of a flux $\mathbf{F}$.
} 
applied to non-conservative systems, as it hinges on the symmetry of the Hessian of the relaxation system (1). However, more general results can be obtained from linear analysis. Yong [70] proved that for $n=N-1$, the subcaracteristic condition is necessary for the linear stability of the equilibrium system. For strictly hyperbolic systems, Solem et al. [60] proved that it is also sufficient. Hence, for strictly hyperbolic relaxation systems where $n=N-1$, the subcharacteristic condition is equivalent to linear stability.

The subcharacteristic condition has been shown to be an important trait of many physical models [6,7,23], since the eigenvalues then have a direct physical interpretation as the characteristic wave speeds of the system. In the context of relaxation models for two-phase flow, the fastest wave speeds are the speeds of pressure waves, which involve the fluid-mechanical speeds of sound. The subcharacteristic condition then implies in particular that the sound speeds of an equilibrium model can never exceed that of the relaxation model it is derived from. This is precisely the observation, well known in the fluid mechanics community, that the "frozen" speed of sound is higher than the equilibrium speed of sound $[22,28,54]$.

\subsection{The model hierarchy}

In a general averaged two-phase flow model, the mixture will consist of two fluids which evolve independently. We assume local thermodynamic equilibrium in each phase, i.e. each of the phases may be described by an equation of state. Specifying two thermodynamic quantities then completely determines all thermodynamic properties of that phase. Herein lies also the assumption that the thermodynamic quantities are unaffected by the local velocity field. Each phase $k$ may then be thought of as having separate pressures $p_{k}$, temperatures $T_{k}$, chemical potentials $\mu_{k}$, and velocities $v_{k}$. Since the two-phase mixture will move towards phase equilibrium in each of the mentioned variables, we may model these interactions by employing relaxation source terms corresponding to the following relaxation processes:

$p$ - volume transfer. Relaxation towards mechanical equilibrium due to pressure differences between the phases, i.e. expansion or compression.

$T$ - heat transfer. Relaxation towards thermal equilibrium, due to temperature differences between the phases.

$\mu$ - mass transfer. Relaxation towards chemical equilibrium due to differences between the phases in chemical potential. ${ }^{2}$

$v$ - momentum transfer. Relaxation towards velocity equilibrium, due to velocity differences between the phases, i.e. interface friction.

The starting point of the forthcoming analysis will be the classical Baer-Nunziato (BN) model [4], which is a general formulation of a two-fluid model, in the sense that the phases are associated with separate velocity fields. The BN model is endowed with appropriate relaxation terms corresponding to each of these processes presented above. By considering the homogeneous and equilibrium limits of each relaxation process, i.e. assuming all combinations of zero or more of them to be instantaneous, we obtain a hierarchy of models, each with partial equilibrium in one or more of the aforementioned variables.

This hierarchy can be represented as a four-dimensional hypercube, as illustrated in Figure 1. Herein, each model is symbolized by a circle, and corresponds to a "corner" of the hypercube. Parallel edges, in turn, correspond to the same instantaneous relaxation assumption, in the direction of the arrow. The basic model, denoted by " 0 " and shown in red as the leftmost circle of Figure 1, is thus reducible to all models in the hierarchy. Many of the models in the hierarchy have already been derived, explicitly expressed and thoroughly analyzed, and in this respect, the current paper builds heavily on previous work $[2,8,15,25,35,37,58,59,71]$.

The models shown in yellow circles in Figure 1 constitute the $v$-branch of the hierarchy, i.e. the homogeneous flow models, wherein the phase velocities are equal. Such models are a subclass of the so-called drift-flux models, where the phasic velocities are related by an algebraic expression. Herein, the $v$-model was derived by [59], the $v p$-model is due to [35] (see also Refs. [2,49]), and the $v p T$-model was studied e.g. by [23]. The $v p T \mu$-model is known as the homogeneous equilibrium model and has been studied by several authors, see e.g.

\footnotetext{
${ }^{2}$ See also Remark 6.1.
} 


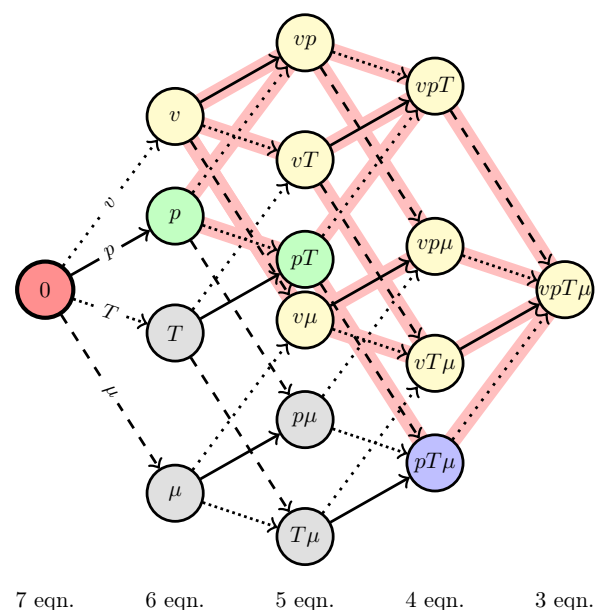

Figure 1. The 4-dimensional hypercube representing the model hierarchy. Parallel edges correspond to the same relaxation processes, and each vertex signifies a unique model in the hierarchy, assuming instantaneous relaxation in zero or more of the variables $p$ (pressure), $T$ (temperature), $\mu$ (chemical potential) and $v$ (velocity). The leftmost, red circle denoted by "0" represents the Baer-Nunziato model [4]. The colored edges represent relaxation processes where a subcharacteristic condition has previously been explicitly established in the literature; models described in [22] and [42] are shown in yellow, whereas models described by [21] are shown in green. Subcharacteristic conditions were obtained in [46] for the model represented by the blue circle.

Refs. [13, 20,32,33,44,62,67]. Flåtten and Lund [22] collected results on the $v$-, vp-, vpT-, and $v p T \mu$-models, derived the $v p \mu$-model, and showed that the subcharacteristic condition was satisfied for all relaxation processes within this branch of the hierarchy. Lund [42] completed the $v$-hierarchy by deriving the $v T$-, $v \mu$ - and $v T \mu$ models, and established the subcharacteristic condition in the remainder of the $v$-branch, given only physically fundamental assumptions.

With regards to the two-fluid models in the hierarchy, several of these models have been derived, employed in simulation $[9,11]$, and analyzed. Here, the $p$-model was analyzed e.g. in Refs. [14,62], and the $p T$-model was studied e.g. in Refs. [21, 29].

An important issue with $p$-relaxed (one-pressure) two-fluid models is that they develop complex eigenvalues when the velocity difference between the phases exceeds a critical value, i.e. they become non-hyperbolic $[17$, $24,46,62,66$. This obviously entails a violation of the subcharacteristic condition, and may lead to the lack of stable mathematical and numerical solutions. Nevertheless, these models are extensively used for practical applications; and in numerical simulations they are often mitigated by specifying a regularizing interfacial pressure (see $[11,51,63])$. Further, estimates of fluid-mechanical sound speeds is of practical importance for the construction of efficient numerical schemes [29,55]. For relations between two-fluid models, we therefore find, as in Ref. [21], the need to state a weaker formulation of the subcharacteristic condition.

Definition 1.2 (Weak subcharacteristic condition). When the subcharacteristic condition of Definition 1.1 holds with the additional equilibrium condition of equal phasic velocities, the weak subcharacteristic condition is said to be satisfied.

Note that even in the case of equal phasic velocites, two-fluid models do not generally satisfy the strict hyperbolicity needed for the proof of equivalence between the subcharacteristic condition and linear stability presented in [60]. Hence, this weakening of the condition serves a mainly heuristic purpose. 
The $p$ - and $p T$-models were analyzed by Martínez Ferrer et al. [21], who showed that the subcharacteristic condition, in a weak or strong sense, is satisfied with respect to existing neighbouring models. Similarly, Morin and Flåtten [46] studied the $p T \mu$-model, and showed that subcharacteristic conditions were satisfied in relation to existing neighbouring models. The highlighted edges in Figure 1 summarize the relations between models where a subcharacteristic condition has already been shown to be satisfied.

\subsection{Contributions of this paper}

The objective of the current paper is to complete the study of the subcharacteristic condition in the full hierarchy of two-phase flow models, proving the remaining subcharacteristic conditions. In this respect, a generalization of the work by [22] and [42] is provided, extending the hierarchy to encompass also two-fluid models, i.e. models with separate momentum equations for the two phases. Herein, the inclusion of the twofluid $T-, \mu^{-}, p \mu$ - and $T \mu$-models represent original contributions. A similar hierarchical derivation of two-phase relaxation models was done in the thesis of Labois [37], who focused primarily on the stiffened gas equationof-state. In our current work, expressions for the sound speeds of the models are provided, valid for general equations of state. Moreover, we show that the remaining 15 subcharactistic conditions are satisfied, i.e. that the subcharacteristic condition is everywhere respected in the hierarchy, either in a strong or in a weak sense. This is done by comparing the new expressions for the sound speeds to many known results from the literature, and by using techniques involving writing the difference of wave velocities as sums of squares (cf. [22,42]). We present each of the models for which we prove at least one subcharacteristic condition.

\subsection{Outline}

The organization of the current paper is as follows. In Section 2 we present the basic model with all possible source terms, derive evolution equations for the primitive variables, and state a parameter set which suffices to satisfy the laws of thermodynamics. In Sections 3 to 8, we present in turn the $v-, p-, T-, \mu-, p \mu-$ and $T \mu-$ models. For each model we give explicit analytic expressions for the sound speeds, and prove the remaining subcharacterisic conditions with respect to related models. In Section 9 we show plots of the sound speeds in the different models, and briefly discuss physical and mathematical properties of models in the hierarchy. Finally, in Section 10, we draw conclusions and suggest possible future work.

\section{BASIC MODEL}

In this section, we present the basic BN model [4]. In this model, which is hyperbolic, the two phases have separate pressures, temperatures, chemical potentials and velocities. We state the model in a form reminiscent of that proposed by Saurel and Abgrall [56], but with all four possible relaxation source terms accounting for the interaction between the phases. From this, we determine the evolution equations of the primitive variables. Based on the evolution equations, we derive a parameter set which suffices for the model to satisfy fundamental physical laws.

\subsection{Governing equations}

In the following, we present the governing equations in the basic model, supplemented with physically appropriate relaxation terms. We let $\alpha_{k}$ denote volume fraction, $v_{k}$ velocity, $\rho_{k}$ density, $p_{k}$ pressure, $T_{k}$ temperature,

$\mu_{k}$ chemical potential, $e_{k}$ internal energy per mass, for each phase $k \in\{\mathrm{g}, \ell\}$, where g denotes gas and $\ell$ denotes liquid.

\subsubsection{Volume advection}

We assume that apart from advection, the interface between the phases can only move due to pressure differences. This is commonly formulated as

$$
\partial_{t} \alpha_{\mathrm{g}}+v_{\mathrm{i}} \partial_{x} \alpha_{\mathrm{g}}=\mathscr{I}\left(p_{\mathrm{g}}-p_{\ell}\right)
$$


wherein $v_{\mathrm{i}}$ is an interface velocity and $\mathscr{I}$ is the pressure relaxation parameter. Hence, the volume fraction is advected with the velocity $v_{\mathrm{i}}$. There are several discussions available on how to choose this interface velocity, see e.g. [15, 57]. In Section 2.3 .2 we propose to model it using a temperature-dependent average of the phasic velocites, derived from the second law of thermodynamics.

The local volume transfer must occur so that the phase with the lowest pressure is compressed, and the phase with the highest pressure is expanded. This is enforced through $\mathscr{I} \geq 0$. Moreover, the volume fractions must satisfy $\alpha_{\mathrm{g}}+\alpha_{\ell}=1$, where $\alpha_{k} \in(0,1)$, and hence only one evolution equation for the volume fractions is needed.

\subsubsection{Mass balance}

The evolution of the mass of each phase is contained in the balance equations

$$
\begin{gathered}
\partial_{t} \alpha_{\mathrm{g}} \rho_{\mathrm{g}}+\partial_{x} \alpha_{\mathrm{g}} \rho_{\mathrm{g}} v_{\mathrm{g}}=\mathscr{K}\left(\mu_{\ell}-\mu_{\mathrm{g}}\right), \\
\partial_{t} \alpha_{\ell} \rho_{\ell}+\partial_{x} \alpha_{\ell} \rho_{\ell} v_{\ell}=\mathscr{K}\left(\mu_{\mathrm{g}}-\mu_{\ell}\right),
\end{gathered}
$$

wherein $\mathscr{K}$ is the mass relaxation parameter, and the source terms on the right hand sides of (7) and (8) account for mass transfer between the phases [26,27]. The mass transfer occurs from the phase with the highest chemical potential towards the phase with the lowest, which is ensured through the assumption $\mathscr{K} \geq 0$. We observe that conservation of total mass is contained by summing (7) and (8):

$$
\partial_{t} \rho+\partial_{x}\left(\alpha_{\mathrm{g}} \rho_{\mathrm{g}} v_{\mathrm{g}}+\alpha_{\ell} \rho_{\ell} v_{\ell}\right)=0
$$

wherein we have defined the mixture density $\rho=\alpha_{\mathrm{g}} \rho_{\mathrm{g}}+\alpha_{\ell} \rho_{\ell}$.

\subsubsection{Momentum balance}

Similar balance laws apply for the momentum of each phase:

$$
\begin{aligned}
\partial_{t} \alpha_{\mathrm{g}} \rho_{\mathrm{g}} v_{\mathrm{g}}+\partial_{x}\left(\alpha_{\mathrm{g}} \rho_{\mathrm{g}} v_{\mathrm{g}}^{2}+\alpha_{\mathrm{g}} p_{\mathrm{g}}\right)-p_{\mathrm{i}} \partial_{x} \alpha_{\mathrm{g}}=v_{\mathrm{i}} \mathscr{K}\left(\mu_{\ell}-\mu_{\mathrm{g}}\right)+\mathscr{M}\left(v_{\ell}-v_{\mathrm{g}}\right), \\
\partial_{t} \alpha_{\ell} \rho_{\ell} v_{\ell}+\partial_{x}\left(\alpha_{\ell} \rho_{\ell} v_{\ell}^{2}+\alpha_{\ell} p_{\ell}\right)-p_{\mathrm{i}} \partial_{x} \alpha_{\ell}=v_{\mathrm{i}} \mathscr{K}\left(\mu_{\mathrm{g}}-\mu_{\ell}\right)+\mathscr{M}\left(v_{\mathrm{g}}-v_{\ell}\right) .
\end{aligned}
$$

Herein, $p_{\mathrm{i}}$ is an interface pressure and $\mathscr{M}$ is the momentum relaxation parameter. In Section 2.3.2, we propose a thermodynamically consistent expression for $p_{\mathrm{i}}$, given as a temperature-dependent average of the phasic pressures.

Note that from the averaging procedure resulting in these models, the interface velocity $v_{\mathrm{i}}$ in (10) and (11) need not be the same as that in (6) (see e.g. Ref. [45]). However, we have chosen these to be equal to keep the notation to a minimum, as this will not influence the main conclusions of this paper. The source terms associated with $v_{\mathrm{i}}$ on the right hand sides of (10) and (11) represent the momentum of the condensating or vaporizing fluid, which is transferred to the other phase. The source terms associated with $\mathscr{M}$ represent interfacial friction, and are assumed to cause momentum transfer from the phase with highest velocity towards the one with lowest velocity, which is ensured by requiring $\mathscr{M} \geq 0$. We observe that conservation of total momentum is ensured by summing (10) and (11):

$$
\partial_{t}\left(\alpha_{\mathrm{g}} \rho_{\mathrm{g}} v_{\mathrm{g}}+\alpha_{\ell} \rho_{\ell} v_{\ell}\right)+\partial_{x}\left(\alpha_{\mathrm{g}} \rho_{\mathrm{g}} v_{\mathrm{g}}^{2}+\alpha_{\ell} \rho_{\ell} v_{\ell}^{2}+\alpha_{\mathrm{g}} p_{\mathrm{g}}+\alpha_{\ell} p_{\ell}\right)=0
$$

\subsubsection{Energy balance}

The balance laws for the energy of each phase may be stated as

$$
\begin{aligned}
\partial_{t} E_{\mathrm{g}}+\partial_{x}\left(E_{\mathrm{g}} v_{\mathrm{g}}+\alpha_{\mathrm{g}} v_{\mathrm{g}} p_{\mathrm{g}}\right) & -p_{\mathrm{i}} v_{\mathrm{i}} \partial_{x} \alpha_{\mathrm{g}} \\
& =-p_{\mathrm{i}} \mathscr{I}\left(p_{\mathrm{g}}-p_{\ell}\right)+\left(\mu_{\mathrm{i}}+\frac{1}{2} v_{\mathrm{i}}^{2}\right) \mathscr{K}\left(\mu_{\ell}-\mu_{\mathrm{g}}\right)+v_{\mathrm{i}} \mathscr{M}\left(v_{\ell}-v_{\mathrm{g}}\right)+\mathscr{H}\left(T_{\ell}-T_{\mathrm{g}}\right),
\end{aligned}
$$




$$
\begin{aligned}
\partial_{t} E_{\ell}+\partial_{x}\left(E_{\ell} v_{\ell}+\alpha_{\ell} v_{\ell} p_{\ell}\right) & -p_{\mathrm{i}} v_{\mathrm{i}} \partial_{x} \alpha_{\ell} \\
& =-p_{\mathrm{i}} \mathscr{I}\left(p_{\ell}-p_{\mathrm{g}}\right)+\left(\mu_{\mathrm{i}}+\frac{1}{2} v_{\mathrm{i}}^{2}\right) \mathscr{K}\left(\mu_{\mathrm{g}}-\mu_{\ell}\right)+v_{\mathrm{i}} \mathscr{M}\left(v_{\mathrm{g}}-v_{\ell}\right)+\mathscr{H}\left(T_{\mathrm{g}}-T_{\ell}\right) .
\end{aligned}
$$

Herein, $\mu_{\mathrm{i}}$ is an interface chemical potential, $\mathscr{H}$ is the temperature relaxation parameter, and we have introduced the total phasic energy per volume $E_{k}=E_{k}^{\text {int }}+E_{k}^{\text {kin }}$, where the phasic internal and kinetic energies are given by, respectively,

$$
\begin{aligned}
& E_{k}^{\mathrm{int}}=\alpha_{k} \rho_{k} e_{k}, \\
& E_{k}^{\mathrm{kin}}=\frac{1}{2} \alpha_{k} \rho_{k} v_{k}^{2} .
\end{aligned}
$$

On the right hand side of (13) and (14), the terms associated with $\mathscr{I}$ represent energy transfer due to expansioncompression work, the terms associated with $\mathscr{K}$ represent the energy which the condensating or vaporizing fluid brings into the other phase, the terms associated with $\mathscr{M}$ represent energy transfer due to frictious momentum transfer, and the terms associated with $\mathscr{H}$ represent pure heat flow. The latter should flow from the hotter to the colder phase, which is ensured through the assumption $\mathscr{H} \geq 0$. Moreover, we see that total energy is conserved by summing (13) and (14),

$$
\partial_{t} E+\partial_{x}\left(E_{\mathrm{g}} v_{\mathrm{g}}+E_{\ell} v_{\ell}+\alpha_{\mathrm{g}} v_{\mathrm{g}} p_{\mathrm{g}}+\alpha_{\ell} v_{\ell} p_{\ell}\right)=0,
$$

where we have introduced the mixed total energy per volume $E=E_{\mathrm{g}}+E_{\ell}$. Note that the same observation on the interfacial velocity as pointed out in Section 2.1.3 applies to (13) and (14). The interface velocity is for simplicity of notation chosen to be the same $v_{\mathrm{i}}$ in (13) and (14) as in (6) and (10) and (11), but the choice does not have consequences for our main conclusions.

\subsubsection{Phase independent form}

With all possible relaxation terms, the BN model [4], as presented in (6) to (8), (10), (11), (13) and (14), can be stated compactly as

$$
\begin{aligned}
\partial_{t} \alpha_{k}+v_{\mathrm{i}} \partial_{x} \alpha_{k} & =I_{k}, \\
\partial_{t} \alpha_{k} \rho_{k}+\partial_{x} \alpha_{k} \rho_{k} v_{k} & =K_{k}, \\
\partial_{t} \alpha_{k} \rho_{k} v_{k}+\partial_{x}\left(\alpha_{k} \rho_{k} v_{k}^{2}+\alpha_{k} p_{k}\right)-p_{\mathrm{i}} \partial_{x} \alpha_{k} & =v_{\mathrm{i}} K_{k}+M_{k}, \\
\partial_{t} E_{k}+\partial_{x}\left(E_{k} v_{k}+\alpha_{k} v_{k} p_{k}\right)-p_{\mathrm{i}} v_{\mathrm{i}} \partial_{x} \alpha_{k} & =-p_{\mathrm{i}} I_{k}+\left(\mu_{\mathrm{i}}+\frac{1}{2} v_{\mathrm{i}}^{2}\right) K_{k}+v_{\mathrm{i}} M_{k}+H_{k},
\end{aligned}
$$

for each phase $k \in\{\mathrm{g}, \ell\}$. Herein, the shorthand forms of the relaxation source terms, $I_{k}, K_{k}, H_{k}$ and $M_{k}$, have been defined such that $I_{\mathrm{g}}=-I_{\ell}=\mathscr{I}\left(p_{\mathrm{g}}-p_{\ell}\right), K_{\mathrm{g}}=-K_{\ell}=\mathscr{K}\left(\mu_{\ell}-\mu_{\mathrm{g}}\right), H_{\mathrm{g}}=-H_{\ell}=\mathscr{H}\left(T_{\ell}-T_{\mathrm{g}}\right)$, and $M_{\mathrm{g}}=-M_{\ell}=\mathscr{M}\left(v_{\ell}-v_{\mathrm{g}}\right)$.

\subsection{Evolution of primitive variables}

In order to systematically derive other models in the hierarchy, and to derive a physically valid parameter set for the basic model, we now seek the evolution equations for primitive variables, such as phasic velocity $v_{k}$, density $\rho_{k}$, pressure $p_{k}$, temperature $T_{k}$, entropy $s_{k}$ and chemical potential $\mu_{k}$. To simplify the notation in the forthcoming, we introduce the phasic material derivative, defined by

$$
\mathrm{D}_{k}(\cdot) \equiv \partial_{t}(\cdot)+v_{k} \partial_{x}(\cdot),
$$

for each phase $k \in\{\mathrm{g}, \ell\}$. 
In the forthcoming calculations, the following relation will prove useful. For an arbitrary quantity $f$, we have from (19) and (22) that

$$
\alpha_{k} \rho_{k} \mathrm{D}_{k} f=\partial_{t} \alpha_{k} \rho_{k} f+\partial_{x} \alpha_{k} \rho_{k} v_{k} f-f K_{k}
$$

\subsubsection{Volume fraction}

For clarity we state the evolution equation for the volume fraction. Using (18), we have that

$$
\mathrm{D}_{k} \alpha_{k}=I_{k}+\left(v_{k}-v_{\mathrm{i}}\right) \partial_{x} \alpha_{k}
$$

\subsubsection{Velocity}

We now seek the evolution equation for the phasic velocity. Using $f=v_{k}$ in (23), and (20), we obtain

$$
\mathrm{D}_{k} v_{k}=\left(\alpha_{k} \rho_{k}\right)^{-1}\left(\left(p_{\mathrm{i}}-p_{k}\right) \partial_{x} \alpha_{k}-\alpha_{k} \partial_{x} p_{k}+\left(v_{\mathrm{i}}-v_{k}\right) K_{k}+M_{k}\right)
$$

\subsubsection{Density}

The density evolution equation is found by combining (19) and (24),

$$
\mathrm{D}_{k} \rho_{k}=-\frac{\rho_{k}}{\alpha_{k}}\left(v_{k}-v_{\mathrm{i}}\right) \partial_{x} \alpha_{k}-\rho_{k} \partial_{x} v_{k}-\frac{\rho_{k}}{\alpha_{k}} I_{k}+\frac{1}{\alpha_{k}} K_{k} .
$$

\subsubsection{Kinetic energy}

In order to obtain the evolution equation for the specific internal energy, we start by finding the evolution equations for the kinetic energy. Using $f=v_{k}^{2} / 2$ in (23), and (16) and (25), we obtain

$$
\partial_{t} E_{k}^{\mathrm{kin}}+\partial_{x} E_{k}^{\mathrm{kin}} v_{k}+\alpha_{k} v_{k} \partial_{x} p_{k}+v_{k}\left(p_{k}-p_{\mathrm{i}}\right) \partial_{x} \alpha_{k}=\left(v_{\mathrm{i}} v_{k}-\frac{1}{2} v_{k}^{2}\right) K_{k}+v_{k} M_{k} .
$$

\subsubsection{Internal energy}

We obtain the evolution equation for the internal energy by subtracting (27) from (21), expanding and collecting terms:

$$
\partial_{t} E_{k}^{\mathrm{int}}+\partial_{x} E_{k}^{\mathrm{int}} v_{k}+\alpha_{k} p_{k} \partial_{x} v_{k}+p_{\mathrm{i}}\left(v_{k}-v_{\mathrm{i}}\right) \partial_{x} \alpha_{k}=-p_{\mathrm{i}} I_{k}+g_{k} K_{k}+\left(v_{\mathrm{i}}-v_{k}\right) M_{k}+H_{k},
$$

where we have introduced a shorthand interface energy $g_{k}=\mu_{\mathrm{i}}+\frac{1}{2}\left(v_{\mathrm{i}}-v_{k}\right)^{2}$. Now, by using (15) and (28) and $f=e_{k}$ in $(23)$, we obtain

$$
\mathrm{D}_{k} e_{k}=\frac{1}{\alpha_{k} \rho_{k}}\left(-p_{\mathrm{i}}\left(I_{k}+\left(v_{k}-v_{\mathrm{i}}\right) \partial_{x} \alpha_{k}\right)-\alpha_{k} p_{k} \partial_{x} v_{k}+\left(g_{k}-e_{k}\right) K_{k}+\left(v_{\mathrm{i}}-v_{k}\right) M_{k}+H_{k}\right) .
$$

\subsubsection{Entropy}

The fundamental thermodynamic differential reads

$$
\mathrm{d} e_{k}=T_{k} \mathrm{~d} s_{k}+p_{k} \rho_{k}^{-2} \mathrm{~d} \rho_{k},
$$

where $s_{k}$ is the specific entropy of phase $k$. By writing (30) in terms of material derivatives, and inserting (26) and (29), we obtain the evolution equation for the phasic entropy as

$$
\mathrm{D}_{k} s_{k}=\left(\alpha_{k} \rho_{k} T_{k}\right)^{-1}\left[\left(p_{k}-p_{\mathrm{i}}\right)\left(I_{k}+\left(v_{k}-v_{\mathrm{i}}\right) \partial_{x} \alpha_{k}\right)+\left(g_{k}-h_{k}\right) K_{k}+\left(v_{\mathrm{i}}-v_{k}\right) M_{k}+H_{k}\right] .
$$

Herein, the phasic specific enthalpy is defined as $h_{k}=e_{k}+p_{k} / \rho_{k}$. By using $f=s_{k}$ in (23) along with the identity $\mu_{k}=h_{k}-T_{k} s_{k}$, (31) may be written in the balance form

$$
\partial_{t} S_{k}+\partial_{x} S_{k} v_{k}=T_{k}^{-1}\left[\left(p_{k}-p_{\mathrm{i}}\right)\left(I_{k}+\left(v_{k}-v_{\mathrm{i}}\right) \partial_{x} \alpha_{k}\right)+\left(g_{k}-\mu_{k}\right) K_{k}+\left(v_{\mathrm{i}}-v_{k}\right) M_{k}+H_{k}\right]
$$


where we have defined the phasic entropy per volume $S_{k}=\alpha_{k} \rho_{k} s_{k}$.

\subsubsection{Pressure}

The pressure differential in terms of the density and entropy differentials may be written as

$$
\mathrm{d} p_{k}=c_{k}^{2} \mathrm{~d} \rho_{k}+\Gamma_{k} \rho_{k} T_{k} \mathrm{~d} s_{k},
$$

where we have introduced the phasic thermodynamic speed of sound and the first Grüneisen coefficient, respectively defined by

$$
c_{k}^{2}=\left(\partial p_{k} / \partial \rho_{k}\right)_{s_{k}} \quad \text { and } \quad \Gamma_{k}=\rho_{k}^{-1}\left(\partial p_{k} / \partial e_{k}\right)_{\rho_{k}} .
$$

By writing (33) in terms of the phasic material derivative, and inserting (26) and (29), we arrive at

$$
\mathrm{D}_{k} p_{k}=\frac{\Gamma_{k}\left(p_{k}-p_{\mathrm{i}}\right)-\rho_{k} c_{k}^{2}}{\alpha_{k}}\left(I_{k}+\left(v_{k}-v_{\mathrm{i}}\right) \partial_{x} \alpha_{k}\right)-\rho_{k} c_{k}^{2} \partial_{x} v_{k}+\frac{\Gamma_{k}\left(g_{k}-h_{k}\right)+c_{k}^{2}}{\alpha_{k}} K_{k}+\frac{\Gamma_{k}}{\alpha_{k}}\left(v_{\mathrm{i}}-v_{k}\right) M_{k}+\frac{\Gamma_{k}}{\alpha_{k}} H_{k} .
$$

\subsubsection{Temperature}

We now seek the equation governing the phasic temperature evolution. The temperature differential may in terms of the pressure and entropy differentials be written as

$$
\mathrm{d} T_{k}=\Gamma_{k} T_{k} \rho_{k}^{-1} c_{k}^{-2} \mathrm{~d} p_{k}+T_{k} C_{p, k}^{-1} \mathrm{~d} s_{k},
$$

where the specific isobaric heat capacity is defined by $C_{p, k}=T_{k}\left(\partial s_{k} / \partial T_{k}\right)_{p_{k}}$. Now, writing (36) in terms of phasic material derivatives, and inserting (31) and (35), we obtain

$$
\begin{aligned}
\mathrm{D}_{k} T_{k}=\left[\frac { 1 + \frac { \Gamma _ { k } ^ { 2 } C _ { p , k } T _ { k } } { c _ { k } ^ { 2 } } } { \alpha _ { k } \rho _ { k } C _ { p , k } } \left(p_{k}\right.\right. & \left.\left.-p_{\mathrm{i}}\right)-\frac{\Gamma_{k} T_{k}}{\alpha_{k}}\right]\left(I_{k}+\left(v_{k}-v_{\mathrm{i}}\right) \partial_{x} \alpha_{k}\right)-\Gamma_{k} T_{k} \partial_{x} v_{k} \\
+ & {\left[\frac{\Gamma_{k} T_{k}}{\alpha_{k} \rho_{k}}+\frac{1+\frac{\Gamma_{k}^{2} C_{p, k} T_{k}}{c_{k}^{2}}}{\alpha_{k} \rho_{k} C_{p, k}}\left(g_{k}-h_{k}\right)\right] K_{k}+\frac{1+\frac{\Gamma_{k}^{2} C_{p, k} T_{k}}{c_{k}^{2}}}{\alpha_{k} \rho_{k} C_{p, k}}\left[\left(v_{\mathrm{i}}-v_{k}\right) M_{k}+H_{k}\right] . }
\end{aligned}
$$

\subsubsection{Chemical potential}

The natural differential of the phasic chemical potential reads

$$
\mathrm{d} \mu_{k}=\rho_{k}^{-1} \mathrm{~d} p_{k}-s_{k} \mathrm{~d} T_{k} .
$$

Therefore, writing (38) in terms of phasic material derivatives, and inserting (35) and (37), we obtain

$$
\begin{aligned}
\mathrm{D}_{k} \mu_{k}=\frac{1}{\alpha_{k}}[ & \left.\left(\Gamma_{k}-\frac{s_{k}}{C_{p, k}}-\frac{\Gamma_{k}^{2} T_{k} s_{k}}{c_{k}^{2}}\right) \frac{\left(p_{k}-p_{\mathrm{i}}\right)}{\rho_{k}}-c_{k}^{2}+\Gamma_{k} T_{k} s_{k}\right]\left(I_{k}+\left(v_{k}-v_{\mathrm{i}}\right) \partial_{x} \alpha_{k}\right) \\
& -\left(c_{k}^{2}-\Gamma_{k} T_{k} s_{k}\right) \partial_{x} v_{k}+\frac{1}{\alpha_{k} \rho_{k}}\left[c_{k}^{2}-\Gamma_{k} T_{k} s_{k}+\left(\Gamma_{k}-\frac{s_{k}}{C_{p, k}}-\frac{\Gamma_{k}^{2} T_{k} s_{k}}{c_{k}^{2}}\right)\left(g_{k}-h_{k}\right)\right] K_{k} \\
& +\frac{1}{\alpha_{k} \rho_{k}}\left(\Gamma_{k}-\frac{s_{k}}{C_{p, k}}-\frac{\Gamma_{k}^{2} T_{k} s_{k}}{c_{k}^{2}}\right)\left[\left(v_{\mathrm{i}}-v_{k}\right) M_{k}+H_{k}\right] .
\end{aligned}
$$

\subsection{Laws of thermodynamics}

For the model to correctly represent physical phenomena, it should be verified that it satisfies fundamental physical principles [22,23]. We have already verified that it conserves mass, momentum and energy, respectively represented by (9), (12) and (17), where the latter is known as the first law of thermodynamics. We now consider the second law of thermodynamics, which states that the total entropy should be non-decreasing. The analysis in the following is reminiscent of that of various previous works [15, 22]. 


\subsubsection{Total entropy evolution}

The total entropy per volume is given by $S=S_{\mathrm{g}}+S_{\ell}$. The evolution equation for the total entropy is therefore found by summing (32) over $k \in\{\mathrm{g}, \ell\}$ :

$$
\partial_{t} S+\partial_{x}\left(S_{\mathrm{g}} v_{\mathrm{g}}+S_{\ell} v_{\ell}\right)=\mathscr{S}_{p}+\mathscr{S}_{\mu}+\mathscr{S}_{v}+\mathscr{S}_{T}=\mathscr{S}
$$

where we have defined the entropy source terms

$$
\begin{aligned}
& \mathscr{S}_{p}=\left(\frac{p_{\mathrm{g}}-p_{\mathrm{i}}}{T_{\mathrm{g}}}-\frac{p_{\ell}-p_{\mathrm{i}}}{T_{\ell}}\right) I_{\mathrm{g}}+\left[\frac{\left(p_{\mathrm{g}}-p_{\mathrm{i}}\right)\left(v_{\mathrm{g}}-v_{\mathrm{i}}\right)}{T_{\mathrm{g}}}-\frac{\left(p_{\ell}-p_{\mathrm{i}}\right)\left(v_{\ell}-v_{\mathrm{i}}\right)}{T_{\ell}}\right] \partial_{x} \alpha_{\mathrm{g}}, \\
& \mathscr{S}_{\mu}=\left(\left(\mu_{\mathrm{i}}-\mu_{\mathrm{g}}+\frac{1}{2}\left(v_{\mathrm{i}}-v_{\mathrm{g}}\right)^{2}\right) T_{\mathrm{g}}^{-1}-\left(\mu_{\mathrm{i}}-\mu_{\ell}+\frac{1}{2}\left(v_{\mathrm{i}}-v_{\ell}\right)^{2}\right) T_{\ell}^{-1}\right) K_{\mathrm{g}}, \\
& \mathscr{S}_{v}=\left[\left(v_{\mathrm{i}}-v_{\mathrm{g}}\right) T_{\mathrm{g}}^{-1}-\left(v_{\mathrm{i}}-v_{\ell}\right) T_{\ell}^{-1}\right] M_{\mathrm{g}}, \\
& \mathscr{S}_{T}=\left(T_{\mathrm{g}}^{-1}-T_{\ell}^{-1}\right) H_{\mathrm{g}} .
\end{aligned}
$$

\subsubsection{The second law of thermodynamics}

We define the global entropy as

$$
\Omega(t)=\int_{\mathscr{C}} S(x, t) \mathrm{d} x,
$$

where $\mathscr{C} \subseteq \mathbb{R}$ is some closed region. Note that this is the thermodynamic entropy, and not the mathematical entropy discussed by Chen et al. [12]. These are in general not interchangable, although thermodynamic entropy can in some cases play the role as a mathematical entropy. This relationship is highly relevant for the stability analysis of our two-phase flow relaxation models, and we refer to [16] for some discussions. For our present purpose, we limit our interest to the physical validity of our models, i.e. they should satisfy the second law of thermodynamics, which for our models can be formulated as follows.

Definition 2.1. The second law of thermodynamics states that the global entropy is non-decreasing, i.e.,

$$
\frac{\mathrm{d} \Omega}{\mathrm{d} t} \geq 0 \quad \forall t
$$

in our context.

Proposition 2.2. Sufficient conditions for the relaxation model given by (6) to (8), (10), (11), (13) and (14) to satisfy the second law of thermodynamics (Definition 2.1) are

$$
\begin{gathered}
\mathscr{I}, \mathscr{K}, \mathscr{M}, \mathscr{H} \geq 0, \\
\min \left\{\mu_{\mathrm{g}}, \mu_{\ell}\right\} \leq \mu_{\mathrm{i}} \leq \max \left\{\mu_{\mathrm{g}}, \mu_{\ell}\right\} \\
p_{\mathrm{i}}=\frac{\sqrt{T_{\ell}} p_{\mathrm{g}}+\sqrt{T_{\mathrm{g}}} p_{\ell}}{\sqrt{T_{\mathrm{g}}}+\sqrt{T_{\ell}}}, \\
v_{\mathrm{i}}=\frac{\sqrt{T_{\ell}} v_{\mathrm{g}}+\sqrt{T_{\mathrm{g}}} v_{\ell}}{\sqrt{T_{\mathrm{g}}}+\sqrt{T_{\ell}}}
\end{gathered}
$$

given only the physically fundamental assumption $T_{k} \geq 0$ for $k \in\{\mathrm{g}, \ell\}$.

Proof. By temporal differentiation of (45), in combination with (40) and (46), we obtain

$$
\int_{\mathscr{C}} \mathrm{d} x \mathscr{S}(x, t) \geq 0,
$$


where we have assumed that the entropy flux of (40), $S_{\mathrm{g}} v_{\mathrm{g}}+S_{\ell} v_{\ell}$, vanishes at the boundary of $\mathscr{C}$. For (51) to be satisfied, clearly $\mathscr{S} \geq 0$ is a sufficient criterion, for which statement to hold the non-negativity of all the partial source terms $\mathscr{S}_{p}, \mathscr{S}_{\mu}, \mathscr{S}_{T}$ and $\mathscr{S}_{v}$ is in turn sufficient. We now show this for each of the terms under the conditions of (47) to (50).

Firstly, the conditions (49) and (50) inserted into (41) yields

$$
\mathscr{S}_{p}=\mathscr{I}\left(p_{\mathrm{g}}-p_{\ell}\right)^{2}\left(T_{\mathrm{g}} T_{\ell}\right)^{-1 / 2} \geq 0 .
$$

Now, (48) is equivalent to $\mu_{\mathrm{i}}=\beta_{\mu} \mu_{\mathrm{g}}+\left(1-\beta_{\mu}\right) \mu_{\ell}$, with $\beta_{\mu} \in[0,1]$. Hence, combination of (42) and (50) yields

$$
\mathscr{S}_{\mu}=\mathscr{K}\left(\mu_{\ell}-\mu_{\mathrm{g}}\right)^{2}\left[\left(1-\beta_{\mu}\right) T_{\mathrm{g}}^{-1}+\beta_{\mu} T_{\ell}^{-1}\right] \geq 0 .
$$

Next, (50) inserted into (43) yields

$$
\mathscr{S}_{v}=\mathscr{M}\left(v_{\ell}-v_{\mathrm{g}}\right)^{2}\left(T_{\mathrm{g}} T_{\ell}\right)^{-1 / 2} \geq 0 .
$$

Finally, (44) becomes

$$
\mathscr{S}_{T}=\mathscr{H}\left(T_{\ell}-T_{\mathrm{g}}\right)^{2}\left(T_{\mathrm{g}} T_{\ell}\right)^{-1} \geq 0
$$

and hence all the source terms are non-negative.

Remark 2.3. The interface conditions (49) and (50) are sufficient, not necessary, and the square-root-oftemperature weighted average between the phasic values differs from choices in the literature, e.g. the initial choices by [4]. The reason for this particular weighting is that we enforced the interface velocities in (6), (10), (11), (13) and (14) to be equal. Allowing these to differ would enable other linear combinations of the phasic quantities, which could possibly be more suitable for numerical simulations [57]. These differences, however, do not have implications for the main conclusions of this paper.

\subsection{Wave velocities}

We now consider the homogeneous limit of the BN model, where the source terms $\mathscr{I}, \mathscr{K}, \mathscr{M}, \mathscr{H} \rightarrow 0$. The resulting model has previously been extensively studied by several authors, see e.g. [56,71]. The model has two fluid-mechanical sound speeds; one for each of the phases. The seven wave velocities are given by $\boldsymbol{\lambda}_{0}=\left\{v_{\mathrm{i}}, v_{\mathrm{g}}, v_{\ell}, v_{\mathrm{g}}-c_{\mathrm{g}}, v_{\mathrm{g}}+c_{\mathrm{g}}, v_{\ell}-c_{\ell}, v_{\ell}+c_{\ell}\right\}[56]$.

In typical applications, the flow is subsonic, i.e. $\left|v_{\mathrm{g}}-v_{\ell}\right| \ll c_{\mathrm{g}}, c_{\ell}$ may be a valid approximation. Evaluated in the velocity equilibrium limit, taking $v \equiv v_{\mathrm{g}}=v_{\ell}$, the eigenvalues are, sorted in ascending order,

$$
\boldsymbol{\lambda}_{0}^{(0)}=\left\{v-c_{0,+}, v-c_{0,-}, v, v, v, v+c_{0,-}, v+c_{0,+}\right\}
$$

where we have defined $c_{0,+}=\max \left\{c_{\mathrm{g}}, c_{\ell}\right\}$ and $c_{0,-}=\min \left\{c_{\mathrm{g}}, c_{\ell}\right\}$ as the higher and lower sound speeds, respectively.

\section{THE $v$-MODEL}

We now study the model that arises upon imposing instantaneous equilibrium in velocity, i.e. letting the velocity relaxation parameter $\mathscr{M} \rightarrow \infty$, which we expect corresponds to

$$
v_{\mathrm{g}}=v_{\ell} \equiv v .
$$


Simultaneously, we require the term $M_{\mathrm{g}}=\mathscr{M}\left(v_{\ell}-v_{\mathrm{g}}\right)$ to remain finite. By noting that for a general function $f$, the phasic material derivatives are equal for the two phases, i.e. $\mathrm{D}_{k} f=\partial_{t} f+v \partial_{x} f \equiv \mathrm{D} f$, then the system that results from evaluating (25) for the two phases $k \in\{\mathrm{g}, \ell\}$ can be solved to yield

$$
M_{\mathrm{g}}=\left(Y_{\mathrm{g}} p_{\ell}+Y_{\ell} p_{\mathrm{g}}-p_{\mathrm{i}}\right) \partial_{x} \alpha_{\mathrm{g}}+\alpha_{\mathrm{g}} Y_{\ell} \partial_{x} p_{\mathrm{g}}-\alpha_{\ell} Y_{\mathrm{g}} \partial_{x} p_{\ell}
$$

where we have introduced the phasic mass fractions $Y_{k}=\alpha_{k} \rho_{k} / \rho$. The model that now results from inserting (57) and (58) into the basic model of Section 2, was analyzed by Flåtten and Lund [22,42], as it constitutes the basic model of the $v$-branch of the hierarchy. The model is hyperbolic and has previously been studied by many authors $[35,52,59]$.

\subsection{Wave velocities}

The wave velocities of the velocity equilibrium model, in the homogeneous limit where $\mathscr{I}, \mathscr{K}, \mathscr{H} \rightarrow 0$, are given by [22]

$$
\boldsymbol{\lambda}_{v}=\left\{v-c_{v}, v, v, v, v, v+c_{v}\right\}
$$

Herein, the sound speed of this model is defined by

$$
c_{v}^{2}=Y_{\mathrm{g}} c_{\mathrm{g}}^{2}+Y_{\ell} c_{\ell}^{2} .
$$

Proposition 3.1. The $v$-model satisfies the subcharacteristic condition with respect to the basic model, given only the physically fundamental conditions $\rho_{k}, c_{k}^{2}>0$, for $k \in\{\mathrm{g}, \ell\}$.

Proof. We observe that $Y_{\mathrm{g}}+Y_{\ell}=1$, and due to the given positivity conditions, we have that $Y_{k} \in(0,1)$. Therefore, (60) implies that $\min \left\{c_{\mathrm{g}}, c_{\ell}\right\} \leq c_{v} \leq \max \left\{c_{\mathrm{g}}, c_{\ell}\right\}$. It then follows trivially that the wave velocities of the $v$-model are interlaced in the wave velocities (56) of the basic model evaluated in the velocity equilibrium state (57). Hence, the associated subcharacteristic condition of Definition 1.1 is satisfied.

\section{THE $p$-MODEL}

In this section, we consider the mechanical equilibrium model, which arises when we assume instantaneous mechanical equilibrium in the basic model of Section 2. We let the pressure relaxation parameter $\mathscr{I} \rightarrow \infty$, which we expect to correspond to $p_{\mathrm{g}}=p_{\ell} \equiv p$. Simultaneously, the product $I_{\mathrm{g}}=\mathscr{I}\left(p_{\mathrm{g}}-p_{\ell}\right)$ should remain finite. The mechanical equilibrium model is found by using (35) evaluated for each of the two phases. From this, we may find an expression for $I_{\mathrm{g}}$ without temporal derivatives, and insert it into the basic model of Section 2. The resulting model has been extensively studied previously [21,56]. Like other one-pressure two-fluid models, the model is not hyperbolic.

\subsection{Wave velocities}

We consider now the homogeneous limit, where $\mathscr{K}, \mathscr{M}, \mathscr{H} \rightarrow 0$. The eigenvalues to the lowest order in the small parameter $\varepsilon=v_{\mathrm{g}}-v_{\ell}$, i.e. evaluated in the equilibrium state defined by (57), are given by [21]

$$
\boldsymbol{\lambda}_{p}^{(0)}=\left\{v-c_{p}, v, v, v, v, v+c_{p}\right\}
$$

where the sound speed in the $p$-model is given by

$$
c_{p}^{2}=\left(\frac{\alpha_{\mathrm{g}}}{\rho_{\mathrm{g}}}+\frac{\alpha_{\ell}}{\rho_{\ell}}\right)\left(\frac{\alpha_{\mathrm{g}}}{\rho_{\mathrm{g}} c_{\mathrm{g}}^{2}}+\frac{\alpha_{\ell}}{\rho_{\ell} c_{\ell}^{2}}\right)^{-1} .
$$


Proposition 4.1. The p-model satisfies the weak subcharacteristic condition of Definition 1.2 with respect to the basic model of Section 2, subject only to the physically fundamental conditions $\rho_{k}, c_{k}^{2}>0$, for $k \in\{\mathrm{g}, \ell\}$, in the equilibrium state defined by (57).

Proof. We see from (62) that $c_{p}^{2}$ is a convex combination

$$
c_{p}^{2}=\varphi_{\mathrm{g}} c_{\mathrm{g}}^{2}+\varphi_{\ell} c_{\ell}^{2}, \quad \text { where } \quad \varphi_{k}=\left(\frac{\alpha_{k}}{\rho_{k} c_{k}^{2}}\right)\left(\frac{\alpha_{\mathrm{g}}}{\rho_{\mathrm{g}} c_{\mathrm{g}}^{2}}+\frac{\alpha_{\ell}}{\rho_{\ell} c_{\ell}^{2}}\right)^{-1},
$$

since $\varphi_{\mathrm{g}}+\varphi_{\ell}=1$, and $\varphi_{k} \in(0,1)$, due to the given conditions. This implies that

$$
\min \left\{c_{\mathrm{g}}, c_{\ell}\right\} \leq c_{p} \leq \max \left\{c_{\mathrm{g}}, c_{\ell}\right\}
$$

and hence the weak subcharacteristic condition is fullfilled with respect to the basic model, whose local eigenvalues evaluated in the same state are given by (56).

\section{THE T-MODEL}

In this section, we investigate the thermal-equilibrium model ( $T$-model), which emerges from assuming instantaneous thermal equilibrium in the basic model of Section 2. To this end, we let $\mathscr{H} \rightarrow \infty$ herein, which we expect corresponds to

$$
T_{\mathrm{g}}=T_{\ell} \equiv T
$$

in such a way that $H_{\mathrm{g}}=\mathscr{H}\left(T_{\ell}-T_{\mathrm{g}}\right)$ remains finite. In the following we present the governing equations.

\subsection{Governing equations}

The full $T$-model may be stated as the basic model of Section 2, in which (13) and (14) are replaced by (17) and the thermal equilibrium condition (65).

In order to establish the impact of instantaneous thermal relaxation on the wave velocities, we need to express the model in a quasi-linear form, and thus obtain the velocities as the eigenvalues of the associated Jacobian. This is most easily done by exploiting the primitive variables, which is what we now turn to do.

Firstly, we have that the phasic pressure differential in terms of density and temperature may be written as

$$
\mathrm{d} p_{k}=c_{k}^{2} \zeta_{k}^{-1} \mathrm{~d} \rho_{k}+\Gamma_{k} \rho_{k} C_{p, k} \zeta_{k}^{-1} \mathrm{~d} T .
$$

where we have introduced the ratio of specific heats $\zeta_{k}=1+\Gamma_{k}^{2} C_{p, k} T / c_{k}^{2}$, and used (65). With (66), (25) becomes

$$
\mathrm{D}_{k} v_{k}=\frac{\Delta_{\mathrm{i}} p_{k}}{m_{k}} \partial_{x} \alpha_{k}-\frac{c_{k}^{2}}{\rho_{k} \zeta_{k}} \partial_{x} \rho_{k}-\frac{\Gamma_{k} C_{p, k}}{\zeta_{k}} \partial_{x} T+\frac{\Delta_{\mathrm{i}} v_{k}}{m_{k}} K_{k}+\frac{1}{m_{k}} M_{k},
$$

where we have defined the phasic mass per volume $m_{k}=\alpha_{k} \rho_{k}$, the phasic interface pressure jump $\Delta_{\mathrm{i}} p_{k}=p_{\mathrm{i}}-p_{k}$, and the phasic interface velocity difference $\Delta_{\mathrm{i}} v_{k}=v_{\mathrm{i}}-v_{k}$. Furthermore, (37) becomes

$$
\begin{aligned}
\mathrm{D}_{k} T=-\left[\frac{\zeta_{k} \Delta_{\mathrm{i}} p_{k}}{\tilde{C}_{p, k}}+\frac{\Gamma_{k} T}{\alpha_{k}}\right]\left(I_{k}-\Delta_{\mathrm{i}} v_{k} \partial_{x} \alpha_{k}\right)-\Gamma_{k} T \partial_{x} v_{k} & \\
& +\left[\frac{\Gamma_{k} T}{m_{k}}+\frac{\zeta_{k}}{\tilde{C}_{p, k}}\left(g_{k}-h_{k}\right)\right] K_{k}+\frac{\zeta_{k}}{\tilde{C}_{p, k}} \Delta_{\mathrm{i}} v_{k} M_{k}+\frac{\zeta_{k}}{\widetilde{C}_{p, k}} H_{k},
\end{aligned}
$$

where we have introduced the extensive heat capacity at constant pressure $\tilde{C}_{p, k}=m_{k} C_{p, k}$. We now define the weighting factor $\theta_{k}=\tilde{C}_{p, k} \zeta_{k}^{-1} /\left(\tilde{C}_{p, \mathrm{~g}} \zeta_{\mathrm{g}}^{-1}+\tilde{C}_{p, \ell} \zeta_{\ell}^{-1}\right)$, for which clearly $\theta_{\mathrm{g}}+\theta_{\ell}=1$ and $\theta_{k} \in(0,1)$. Multiplying 
(68) by $\theta_{k}$, and summing over the phases yields

$$
\begin{aligned}
& \partial_{t} T+\left(\theta_{\mathrm{g}} v_{\mathrm{g}}+\right.\left.\theta_{\ell} v_{\ell}\right) \partial_{x} T=-\left[\frac{\theta_{\mathrm{g}} \Gamma_{\mathrm{g}} T}{\alpha_{\mathrm{g}}}+\frac{\theta_{\ell} \Gamma_{\ell} T}{\alpha_{\ell}}\right] \frac{v_{\mathrm{g}}-v_{\ell}}{2} \partial_{x} \alpha_{\mathrm{g}}-\theta_{\mathrm{g}} \Gamma_{\mathrm{g}} T \partial_{x} v_{\mathrm{g}}-\theta_{\ell} \Gamma_{\ell} T \partial_{x} v_{\ell} \\
&+\left[\frac{p_{\mathrm{g}}-p_{\ell}}{\frac{\tilde{C}_{p, \mathrm{~g}}}{\zeta_{\mathrm{g}}}+\frac{\tilde{C}_{p, \ell}}{\zeta_{\ell}}}-\frac{\theta_{\mathrm{g}} \Gamma_{\mathrm{g}} T}{\alpha_{\mathrm{g}}}+\frac{\theta_{\ell} \Gamma_{\ell} T}{\alpha_{\ell}}\right] I_{\mathrm{g}}+\left[\frac{h_{\ell}-h_{\mathrm{g}}}{\frac{\tilde{C}_{p, \mathrm{~g}}}{\zeta_{\mathrm{g}}}+\frac{\tilde{C}_{p, \ell}}{\zeta_{\ell}}}+\frac{\theta_{\mathrm{g}} \Gamma_{\mathrm{g}} T}{m_{\mathrm{g}}}-\frac{\theta_{\ell} \Gamma_{\ell} T}{m_{\ell}}\right] K_{\mathrm{g}} \\
&+\frac{v_{\ell}-v_{\mathrm{g}}}{\frac{\tilde{C}_{p, \mathrm{~g}}}{\zeta_{\mathrm{g}}}+\frac{\tilde{C}_{p, \ell}}{\zeta_{\ell}}} M_{\mathrm{g}},
\end{aligned}
$$

where have used the interface parameter definitions of (49) and (50) evaluated in thermal equilibrium (65) to simplify.

\subsection{Wave velocities}

We now seek the wave velocities, i.e. eigenvalues, in the homogeneous limit, where the relaxation source terms $\mathscr{I}, \mathscr{K}, \mathscr{M} \rightarrow 0$. From $(24)$, it is then clear that $\alpha_{\mathrm{g}}$ is a characteristic variable of the system, since the volume fraction is advected with the velocity $v_{\mathrm{i}}$ in the absence of relaxation source terms. By using (26), (67) and (69), the remaining, reduced system may now be expressed in the quasi-linear form $\partial_{t} \tilde{\mathbf{u}}_{T}+\tilde{\mathbf{A}}_{T}\left(\tilde{\mathbf{u}}_{T}\right) \partial_{x} \tilde{\mathbf{u}}_{T}=0$, where $\tilde{\mathbf{u}}_{T}=\left[\rho_{\mathrm{g}}, \rho_{\ell}, v_{\mathrm{g}}, v_{\ell}, T\right]$, and the associated Jacobian is given by

$$
\tilde{\mathbf{A}}_{T}=\left[\begin{array}{ccccc}
v_{\mathrm{g}} & 0 & \rho_{\mathrm{g}} & 0 & 0 \\
0 & v_{\ell} & 0 & \rho_{\ell} & 0 \\
\frac{c_{\mathrm{g}}^{2}}{\rho_{\mathrm{g}} \zeta_{\mathrm{g}}} & 0 & v_{\mathrm{g}} & 0 & \frac{\Gamma_{\mathrm{g}} C_{p, \mathrm{~g}}}{\zeta_{\mathrm{g}}} \\
0 & \frac{c_{\ell}^{2}}{\rho_{\ell} \zeta_{\ell}} & 0 & v_{\ell} & \frac{\Gamma_{\ell} C_{p, \ell}}{\zeta_{\ell}} \\
0 & 0 & \theta_{\mathrm{g}} \Gamma_{\mathrm{g}} T & \theta_{\ell} \Gamma_{\ell} T & \theta_{\mathrm{g}} v_{\mathrm{g}}+\theta_{\ell} v_{\ell}
\end{array}\right],
$$

from which we can find the remaining five eigenvalues. The characteristic polynomial of the latter is a fifthdegree polynomial, for which in general no closed-form solution can be obtained. We now note that we may write $\tilde{\mathbf{A}}_{T}=\tilde{\mathbf{A}}_{T}^{(0)}+\varepsilon \tilde{\mathbf{A}}_{T}^{(1)}$, where $\varepsilon=v_{\mathrm{g}}-v_{\ell}$. The matrices are given by

$$
\tilde{\mathbf{A}}_{T}^{(0)}=\left[\begin{array}{ccccc}
\bar{v} & 0 & \rho_{\mathrm{g}} & 0 & 0 \\
0 & \bar{v} & 0 & \rho_{\ell} & 0 \\
\frac{c_{\mathrm{g}}^{2}}{\rho_{\mathrm{g}} \zeta_{\mathrm{g}}} & 0 & \bar{v} & 0 & \frac{\Gamma_{\mathrm{g}} C_{p, \mathrm{~g}}}{\zeta_{\mathrm{g}}} \\
0 & \frac{c_{\ell}^{2}}{\rho_{\ell} \zeta_{\ell}} & 0 & \bar{v} & \frac{\Gamma_{\ell} C_{p, \ell}}{\zeta_{\ell}} \\
0 & 0 & \theta_{\mathrm{g}} \Gamma_{\mathrm{g}} T & \theta_{\ell} \Gamma_{\ell} T & \bar{v}
\end{array}\right],
$$

and $\tilde{\mathbf{A}}_{T}^{(1)}=\operatorname{diag}\left(\theta_{\ell},-\theta_{\mathrm{g}}, \theta_{\ell},-\theta_{\mathrm{g}}, 0\right)$, where we have taken $\bar{v}=\theta_{\mathrm{g}} v_{\mathrm{g}}+\theta_{\ell} v_{\ell}$. Hence, we approximate the eigenvalues by means of a perturbation expansion in the small parameter $\varepsilon$. To the lowest order in $\varepsilon, v_{\mathrm{g}}=v_{\ell}=\bar{v}=v$, and the eigenvalues of the $T$-model are given by

$$
\boldsymbol{\lambda}_{T}^{(0)}=\left\{v-c_{T,+}, v-c_{T,-}, v, v, v+c_{T,-}, v+c_{T,+}\right\}
$$

where the two distinct sound speeds of the model are given by

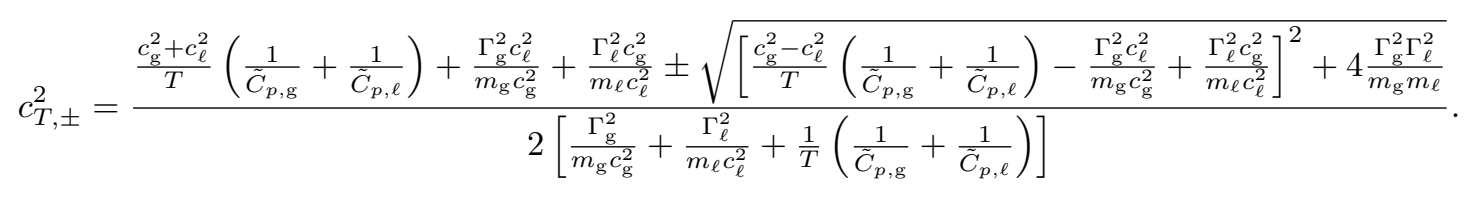


Proposition 5.1. The T-model satisfies the weak subcharacteristic condition with respect to the basic model of Section 2, subject only to the physically fundamental conditions $\rho_{k}, C_{p, k}, T>0$, for $k \in\{\mathrm{g}, \ell\}$, in the equilibrium state defined by (57).

Proof. We first show that the sound speeds are real. We note that on the given conditions, clearly $c_{T, \pm}^{2} \in \mathbb{R}$, and moreover, $c_{T,+}^{2} \geq 0$. The product of the sound speeds may be written as

$$
c_{T,+}^{-2} c_{T,-}^{-2}=c_{0,+}^{-2} c_{0,-}^{-2}+\mathcal{Z}_{T}^{0}, \quad \text { where } \quad \mathcal{Z}_{T}^{0}=\frac{T\left(\frac{\Gamma_{\mathrm{g}}^{2}}{m_{\mathrm{g}} c_{\mathrm{g}}^{2}}+\frac{\Gamma_{\ell}^{2}}{m_{\ell} c_{\ell}^{2}}\right)}{c_{0,+}^{2} c_{0,-}^{2}\left(\frac{1}{\tilde{C}_{p, \mathrm{~g}}}+\frac{1}{\tilde{C}_{p, \ell}}\right)}
$$

Based on the given conditions, it is clear that $\mathcal{Z}_{T}^{0} \geq 0$ and therefore

$$
0 \leq c_{T,+}^{2} c_{T,-}^{2} \leq c_{0,+}^{2} c_{0,-}^{2}
$$

and hence also $c_{T,-}^{2} \geq 0$, and thus $c_{T, \pm}$ are real, and by definition, positive. Now, using the definitions of $c_{0, \pm}$ and (73), it follows that

$$
\left(c_{0,+}^{2}-c_{T,+}^{2}\right)\left(c_{0,+}^{2}-c_{T,-}^{2}\right)\left(c_{0,-}^{2}-c_{T,+}^{2}\right)\left(c_{0,-}^{2}-c_{T,-}^{2}\right)=-\mathcal{Q}_{T}^{0}
$$

where

$$
\mathcal{Q}_{T}^{0}=\left(c_{\mathrm{g}}^{2}-c_{\ell}^{2}\right)^{2} \frac{\Gamma_{\mathrm{g}}^{2} \Gamma_{\ell}^{2}}{m_{\mathrm{g}} m_{\ell}}\left[\frac{\Gamma_{\mathrm{g}}^{2}}{m_{\mathrm{g}} c_{\mathrm{g}}^{2}}+\frac{\Gamma_{\ell}^{2}}{m_{\ell} c_{\ell}^{2}}+\frac{1}{T}\left(\frac{1}{\tilde{C}_{p, \mathrm{~g}}}+\frac{1}{\tilde{C}_{p, \ell}}\right)\right]^{-2}
$$

The given conditions ensure that $\mathcal{Q}_{T}^{0} \geq 0$. The only ordering of sound speeds compatible with (75) and (76) is $0 \leq c_{T,-} \leq c_{0,-} \leq c_{T,+} \leq c_{0,+}$, and hence the subcharacteristic condition of Definition 1.1 is satisfied.

Proposition 5.2. The vT-model of Lund [42] satisfies the subcharacteristic condition with respect to the Tmodel, given the physically fundamental assumptions $\rho_{k}, C_{p, k}, T>0$, for $k \in\{\mathrm{g}, \ell\}$.

Proof. The sound speed of the $v T$-model is given by [42]

$$
c_{v T}^{2}=\frac{1}{\rho} \frac{m_{\mathrm{g}} c_{\mathrm{g}}^{2} m_{\ell} c_{\ell}^{2}\left(\frac{\Gamma_{\mathrm{g}}}{m_{\mathrm{g}} c_{\mathrm{g}}^{2}}+\frac{\Gamma_{\ell}}{m_{\ell} c_{\ell}^{2}}\right)^{2}+\frac{1}{T}\left(\frac{1}{\tilde{C}_{p, \mathrm{~g}}}+\frac{1}{\tilde{C}_{p, \ell}}\right)\left(m_{\mathrm{g}} c_{\mathrm{g}}^{2}+m_{\ell} c_{\ell}^{2}\right)}{\frac{\Gamma_{\mathrm{g}}^{2}}{m_{\mathrm{g}} c_{\mathrm{g}}^{2}}+\frac{\Gamma_{\ell}^{2}}{m_{\ell} c_{\ell}^{2}}+\frac{1}{T}\left(\frac{1}{\tilde{C}_{p, \mathrm{~g}}}+\frac{1}{\tilde{C}_{p, \ell}}\right)} .
$$

Now, using (73), we can write the product of the differences as

$$
\left(c_{T,+}^{2}-c_{v T}^{2}\right)\left(c_{T,-}^{2}-c_{v T}^{2}\right)=-\mathcal{Q}_{v T}^{T}
$$

where

$$
\mathcal{Q}_{v T}^{T}=Y_{\mathrm{g}} Y_{\ell}\left[\frac{\frac{1}{T}\left(\frac{1}{\tilde{C}_{p, \mathrm{~g}}}+\frac{1}{\tilde{C}_{p, \ell}}\right)\left(c_{\mathrm{g}}^{2}-c_{\ell}^{2}\right)-\frac{\Gamma_{\mathrm{g}}^{2} c_{\ell}^{2}}{m_{\mathrm{g}} c_{\mathrm{g}}^{2}}+\frac{\Gamma_{\ell}^{2} c_{\mathrm{g}}^{2}}{m_{\ell} c_{\ell}^{2}}+\left(\frac{1}{m_{\mathrm{g}}}-\frac{1}{m_{\ell}}\right) \Gamma_{\mathrm{g}} \Gamma_{\ell}}{\frac{\Gamma_{\mathrm{g}}^{2}}{m_{\mathrm{g}} c_{\mathrm{g}}^{2}}+\frac{\Gamma_{\ell}^{2}}{m_{\ell} c_{\ell}^{2}}+\frac{1}{T}\left(\frac{1}{\tilde{C}_{p, g}}+\frac{1}{\tilde{C}_{p, \ell}}\right)}\right]^{2} .
$$

With the given conditions, clearly $\mathcal{Q}_{v T}^{T} \geq 0$. Hence exactly one of the factors on the left hand side of (79) is negative, and combined with Proposition 5.1 we realize that $c_{T,-} \leq c_{v T} \leq c_{T,+}$, and hence the subcharacteristic condition is satisfied. 
Proposition 5.3. The $p T$-model satisfies the weak subcharacteristic condition with respect to the T-model, given the physically fundamental assumptions $\rho_{k}, C_{p, k}, T>0$ for $k \in\{\mathrm{g}, \ell\}$ in the equilibrium state defined by (57).

Proof. The sound speed of the $p T$-model is given by [21]

$$
c_{p T}^{2}=\frac{\left(\frac{\alpha_{\mathrm{g}}}{\rho_{\mathrm{g}}}+\frac{\alpha_{\ell}}{\rho_{\ell}}\right)\left(\frac{1}{\tilde{C}_{p, \mathrm{~g}}}+\frac{1}{\tilde{C}_{p, \ell}}\right)}{\left(\frac{\alpha_{\mathrm{g}}}{\rho_{\mathrm{g}} c_{\mathrm{g}}^{2}}+\frac{\alpha_{\ell}}{\rho_{\ell} c_{\ell}^{2}}\right)\left(\frac{1}{\tilde{C}_{p, \mathrm{~g}}}+\frac{1}{\tilde{C}_{p, \ell}}\right)+T\left(\frac{\Gamma_{\mathrm{g}}}{\rho_{\mathrm{g}} c_{\mathrm{g}}^{2}}-\frac{\Gamma_{\ell}}{\rho_{\ell} c_{\ell}^{2}}\right)^{2}}
$$

We may now write

$$
\left(c_{T,+}^{2}-c_{p T}^{2}\right)\left(c_{T,-}^{2}-c_{p T}^{2}\right)=-\mathcal{Q}_{p T}^{T},
$$

where

$$
\begin{aligned}
\mathcal{Q}_{p T}^{T}=\frac{\alpha_{\mathrm{g}} \alpha_{\ell}}{\rho_{\mathrm{g}} c_{\mathrm{g}}^{2} \rho_{\ell} c_{\ell}^{2} T}\left(\frac{1}{\tilde{C}_{p, \mathrm{~g}}}+\frac{1}{\tilde{C}_{p, \ell}}\right)\left[\frac{\Gamma_{\mathrm{g}}^{2}}{m_{\mathrm{g}} c_{\mathrm{g}}^{2}}+\frac{\Gamma_{\ell}^{2}}{m_{\ell} c_{\ell}^{2}}+\frac{1}{T}\left(\frac{1}{\tilde{C}_{p, \mathrm{~g}}}+\frac{1}{\tilde{C}_{p, \ell}}\right)\right]^{-1} \\
\times\left[\frac{\left(\frac{1}{\tilde{C}_{p, \mathrm{~g}}}+\frac{1}{\tilde{C}_{p, \ell}}\right)\left(c_{\mathrm{g}}^{2}-c_{\ell}^{2}\right)-T\left(\frac{\Gamma_{\mathrm{g}}}{\rho_{\mathrm{g}} c_{\mathrm{g}}^{2}}-\frac{\Gamma_{\ell}}{\rho_{\ell} c_{\ell}^{2}}\right)\left(\frac{\Gamma_{\mathrm{g}} c_{\ell}^{2}}{\alpha_{\mathrm{g}}}+\frac{\Gamma_{\ell} c_{\mathrm{g}}^{2}}{\alpha_{\ell}}\right)}{\left(\frac{\alpha_{\mathrm{g}}}{\rho_{\mathrm{g}} c_{\mathrm{g}}^{2}}+\frac{\alpha_{\ell}}{\rho_{\ell} c_{\ell}^{2}}\right)\left(\frac{1}{\tilde{C}_{p, \mathrm{~g}}}+\frac{1}{\tilde{C}_{p, \ell}}\right)+T\left(\frac{\Gamma_{\mathrm{g}}}{\rho_{\mathrm{g}} c_{\mathrm{g}}^{2}}-\frac{\Gamma_{\ell}}{\rho_{\ell} c_{\ell}^{2}}\right)^{2}}\right]
\end{aligned}
$$

Clearly $\mathcal{Q}_{p T}^{T} \geq 0$, on the given conditions. Hence exactly one factor on the left hand side of (82) is negative, yielding $c_{T,-} \leq c_{p T} \leq c_{T,+}$, and the weak subcharacteristic condition is satisfied.

\section{THE $\mu$-MODEL}

We now proceed to investigate the chemical-equilibrium model (the $\mu$-model), which arises when we assume instantaneous chemical equilibrium, i.e. let the chemical relaxation parameter $\mathscr{K} \rightarrow \infty$, which we expect corresponds to

$$
\mu_{\mathrm{g}}=\mu_{\ell} \equiv \mu
$$

Simultaneously, we require the product $K_{\mathrm{g}}=\mathscr{K}\left(\mu_{\ell}-\mu_{\mathrm{g}}\right)$ to remain finite, and in the forthcoming we seek to express this without any temporal derivatives.

Remark 6.1. In our framework $\mathscr{K}$ must be set to zero when transition to one-phase flow occurs. Otherwise, strictly enforcing (84) will lead to unphysical results [3,5,43] in the form of negative volumes. Consequently, we strongly emphasize that all our results concerning nonzero phase transfer are meaningful only for the genuine two-phase region where the flow variables reside on the saturation curve.

With Remark 6.1 in mind, we can write the chemical potential evolution equation (39) as

$$
\mathrm{D}_{k} \mu=-\left[\psi_{k} \Delta_{\mathrm{i}} p_{k}+\xi_{k} \alpha_{k}^{-1}\right]\left(I_{k}-\Delta_{\mathrm{i}} v_{k} \partial_{x} \alpha_{k}\right)-\xi_{k} \partial_{x} v_{k}+\chi_{k} K_{k}+\psi_{k} \Delta_{\mathrm{i}} v_{k} M_{k}+\psi_{k} H_{k} .
$$

where we have used (84), and defined the shorthands

$$
\xi_{k}=c_{k}^{2}-\Gamma_{k} T_{k} s_{k}, \quad \psi_{k}=\frac{\Gamma_{k} \xi_{k}}{m_{k} c_{k}^{2}}-\frac{s_{k}}{\tilde{C}_{p, k}}, \quad \chi_{k}=\frac{\xi_{k}^{2}}{m_{k} c_{k}^{2}}+\frac{T_{k} s_{k}^{2}}{\tilde{C}_{p, k}}+\frac{1}{2}\left(\Delta_{\mathrm{i}} v_{k}\right)^{2} \psi_{k} .
$$


By using (85) evaluated for each of the phases, and subtracting these expressions from each other, we obtain

$$
K_{\mathrm{g}}=\kappa_{\mu}^{-1}\left(\xi_{\mathrm{g}} \partial_{x} v_{\mathrm{g}}-\xi_{\ell} \partial_{x} v_{\ell}-\left(\psi_{\mathrm{g}}+\psi_{\ell}\right) H_{\mathrm{g}}\right)-\mathcal{A}^{\mu} \partial_{x} \alpha_{\mathrm{g}}+\left(v_{\mathrm{g}}-v_{\ell}\right) \kappa_{\mu}^{-1} \partial_{x} \mu+\mathcal{K}_{p}^{\mu} I_{\mathrm{g}}-\mathcal{K}_{v}^{\mu} M_{\mathrm{g}}
$$

where we have defined the shorthands

$$
\begin{aligned}
\kappa_{\mu} & =\frac{T_{\mathrm{g}} s_{\mathrm{g}}^{2}}{\tilde{C}_{p, \mathrm{~g}}}+\frac{T_{\ell} s_{\ell}^{2}}{\tilde{C}_{p, \ell}}+\frac{\xi_{\mathrm{g}}^{2}}{m_{\mathrm{g}} c_{\mathrm{g}}^{2}}+\frac{\xi_{\ell}^{2}}{m_{\ell} c_{\ell}^{2}}+\frac{1}{2}\left(\psi_{\mathrm{g}}\left(\Delta_{\mathrm{i}} v_{\mathrm{g}}\right)^{2}+\psi_{\ell}\left(\Delta_{\mathrm{i}} v_{\ell}\right)^{2}\right) \\
\mathcal{A}^{\mu} & =\kappa_{\mu}^{-1}\left[\left(\psi_{\mathrm{g}} \Delta_{\mathrm{i}} p_{\mathrm{g}}+\frac{\xi_{\mathrm{g}}}{\alpha_{\mathrm{g}}}\right) \Delta_{\mathrm{i}} v_{\mathrm{g}}+\left(\psi_{\ell} \Delta_{\mathrm{i}} p_{\ell}+\frac{\xi_{\ell}}{\alpha_{\ell}}\right) \Delta_{\mathrm{i}} v_{\ell}\right] \\
\mathcal{K}_{p}^{\mu} & =\kappa_{\mu}^{-1}\left[\psi_{\mathrm{g}} \Delta_{\mathrm{i}} p_{\mathrm{g}}+\psi_{\ell} \Delta_{\mathrm{i}} p_{\ell}+\frac{\xi_{\mathrm{g}}}{\alpha_{\mathrm{g}}}+\frac{\xi_{\ell}}{\alpha_{\ell}}\right], \quad \mathcal{K}_{v}^{\mu}=\kappa_{\mu}^{-1}\left(\psi_{\mathrm{g}} \Delta_{\mathrm{i}} v_{\mathrm{g}}+\psi_{\ell} \Delta_{\mathrm{i}} v_{\ell}\right) .
\end{aligned}
$$

\subsection{Governing equations}

By using the expression (87) to insert for $K_{\mathrm{g}}$ in the basic model of Section 2, the $\mu$-model can now be summarized with the following set of equations:

- Volume advection: $\partial_{t} \alpha_{\mathrm{g}}+v_{\mathrm{i}} \partial_{x} \alpha_{\mathrm{g}}=I_{\mathrm{g}}$

- Conservation of mass: $\partial_{t} \rho+\partial_{x}\left(\alpha_{\mathrm{g}} \rho_{\mathrm{g}} v_{\mathrm{g}}+\alpha_{\ell} \rho_{\ell} v_{\ell}\right)=0$,

- Momentum balance:

$$
\begin{aligned}
\partial_{t} \alpha_{\mathrm{g}} \rho_{\mathrm{g}} v_{\mathrm{g}}+\partial_{x}\left(\alpha_{\mathrm{g}} \rho_{\mathrm{g}} v_{\mathrm{g}}^{2}+\alpha_{\mathrm{g}} p_{\mathrm{g}}\right)- & \left(p_{\mathrm{i}}-v_{\mathrm{i}} \mathcal{A}^{\mu}\right) \partial_{x} \alpha_{\mathrm{g}}-v_{\mathrm{i}} \xi_{\mathrm{g}} \kappa_{\mu}^{-1} \partial_{x} v_{\mathrm{g}}+v_{\mathrm{i}} \xi_{\ell} \kappa_{\mu}^{-1} \partial_{x} v_{\ell} \\
& -v_{\mathrm{i}}\left(v_{\mathrm{g}}-v_{\ell}\right) \kappa_{\mu}^{-1} \partial_{x} \mu=v_{\mathrm{i}} \mathcal{K}_{p}^{\mu} I_{\mathrm{g}}+\left(1-v_{\mathrm{i}} \mathcal{K}_{v}^{\mu}\right) M_{\mathrm{g}}-v_{\mathrm{i}}\left(\psi_{\mathrm{g}}+\psi_{\ell}\right) \kappa_{\mu}^{-1} H_{\mathrm{g}}
\end{aligned}
$$

- Energy balance:

$$
\begin{aligned}
& \partial_{t} E_{\mathrm{g}}+\partial_{x}\left(E_{\mathrm{g}} v_{\mathrm{g}}+\alpha_{\mathrm{g}} v_{\mathrm{g}} p_{\mathrm{g}}\right)- {\left[p_{\mathrm{i}} v_{\mathrm{i}}-\left(\mu+\frac{1}{2} v_{\mathrm{i}}^{2}\right) \mathscr{A}^{\mu}\right] \partial_{x} \alpha_{\mathrm{g}} } \\
&-\left(\mu+\frac{1}{2} v_{\mathrm{i}}^{2}\right) \kappa_{\mu}^{-1}\left[\xi_{\mathrm{g}} \partial_{x} v_{\mathrm{g}}-\xi_{\ell} \partial_{x} v_{\ell}+\left(v_{\mathrm{g}}-v_{\ell}\right) \partial_{x} \mu\right] \\
&=\left[\left(\mu+\frac{1}{2} v_{\mathrm{i}}^{2}\right) \mathscr{K}_{p}^{\mu}-p_{\mathrm{i}}\right] I_{\mathrm{g}}+\left[v_{\mathrm{i}}-\left(\mu+\frac{1}{2} v_{\mathrm{i}}^{2}\right) \mathcal{K}_{v}^{\mu}\right] M_{\mathrm{g}}+\left[1-\left(\mu+\frac{1}{2} v_{\mathrm{i}}^{2}\right)\left(\psi_{\mathrm{g}}+\psi_{\ell}\right) \kappa_{\mu}^{-1}\right] H_{\mathrm{g}},
\end{aligned}
$$

Momentum and energy equations for the liquid phase are found by phase symmetry; interchanging indices $g$ and $\ell$.

\subsection{Evolution of primitive variables}

In order to write the system in a quasi-linear form, and thereby find the wave speeds of the $\mu$-model, we use the evolution equations for the primitive variables. We therefore now seek the evolution of some of the primitive variables under the assumption of instantaneous chemical equilibrium.

We first define the weighting factor $\phi_{k}=\chi_{k}^{-1} /\left(\chi_{\mathrm{g}}^{-1}+\chi_{\ell}^{-1}\right)$. Multiplying (85) by $\phi_{k}$ and summing over the phases, we get for the chemical potential

$$
\begin{aligned}
\partial_{t} \mu+\left(\phi_{\mathrm{g}} v_{\mathrm{g}}+\phi_{\ell} v_{\ell}\right) \partial_{x} \mu+ & G_{\alpha_{\mathrm{g}}}^{\mu} \partial_{x} \alpha_{\mathrm{g}}+\phi_{\mathrm{g}} \xi_{\mathrm{g}} \partial_{x} v_{\mathrm{g}}+\phi_{\ell} \xi_{\ell} \partial_{x} v_{\ell} \\
=\left[-\phi_{\mathrm{g}}\left(\psi_{\mathrm{g}} \Delta_{\mathrm{i}} p_{\mathrm{g}}+\xi_{\mathrm{g}} \alpha_{\mathrm{g}}^{-1}\right)\right. & \left.+\phi_{\ell}\left(\psi_{\ell} \Delta_{\mathrm{i}} p_{\ell}+\xi_{\ell} \alpha_{\ell}^{-1}\right)\right] I_{\mathrm{g}} \\
& +\left(\phi_{\mathrm{g}} \psi_{\mathrm{g}} \Delta_{\mathrm{i}} v_{\mathrm{g}}-\phi_{\ell} \psi_{\ell} \Delta_{\mathrm{i}} v_{\ell}\right) M_{\mathrm{g}}+\left(\phi_{\mathrm{g}} \psi_{\mathrm{g}}-\phi_{\ell} \psi_{\ell}\right) H_{\mathrm{g}}
\end{aligned}
$$

where we have defined the shorthand coefficient

$$
G_{\alpha_{\mathrm{g}}}^{\mu}=-\phi_{\mathrm{g}}\left(\psi_{\mathrm{g}} \Delta_{\mathrm{i}} p_{\mathrm{g}}+\xi_{\mathrm{g}} \alpha_{\mathrm{g}}^{-1}\right) \Delta_{\mathrm{i}} v_{\mathrm{g}}+\phi_{\ell}\left(\psi_{\ell} \Delta_{\mathrm{i}} p_{\ell}+\xi_{\ell} \alpha_{\ell}^{-1}\right) \Delta_{\mathrm{i}} v_{\ell}
$$


For the phasic velocity $v_{\mathrm{g}}$, we find from (25) the evolution equation

$$
\begin{array}{r}
\partial_{t} v_{\mathrm{g}}+\left[v_{\mathrm{g}}-\frac{\xi_{\mathrm{g}} \Delta_{\mathrm{i}} v_{\mathrm{g}}}{m_{\mathrm{g}} \kappa_{\mu}}\right] \partial_{x} v_{\mathrm{g}}+\frac{\xi_{\ell} \Delta_{\mathrm{i}} v_{\mathrm{g}}}{m_{\mathrm{g}} \kappa_{\mu}} \partial_{x} v_{\ell}+\frac{\Delta_{\mathrm{i}} v_{\mathrm{g}} \mathcal{A}^{\mu}-\Delta_{\mathrm{i}} p_{\mathrm{g}}}{m_{\mathrm{g}}} \partial_{x} \alpha_{\mathrm{g}}+\frac{1}{\rho_{\mathrm{g}}} \partial_{x} p_{\mathrm{g}}-\frac{\Delta_{\mathrm{i}} v_{\mathrm{g}}\left(v_{\mathrm{g}}-v_{\ell}\right)}{m_{\mathrm{g}} \kappa_{\mu}} \partial_{x} \mu \\
=\frac{\Delta_{\mathrm{i}} v_{\mathrm{g}}}{m_{\mathrm{g}}} \mathcal{K}_{p}^{\mu} I_{\mathrm{g}}+\frac{1}{m_{\mathrm{g}}}\left(1-\Delta_{\mathrm{i}} v_{\mathrm{g}} \mathcal{K}_{v}^{\mu}\right) M_{\mathrm{g}}-\frac{\Delta_{\mathrm{i}} v_{\mathrm{g}}}{m_{\mathrm{g}}} \frac{\psi_{\mathrm{g}}+\psi_{\ell}}{\kappa_{\mu}} H_{\mathrm{g}}
\end{array}
$$

and $v_{\ell}$ is found by phase symmetry.

The phasic pressure evolution is found from (35). For the gas phase, it reads

$$
\begin{aligned}
\partial_{t} p_{\mathrm{g}}+v_{\mathrm{g}} \partial_{x} p_{\mathrm{g}}+P_{\mathrm{g}, \alpha_{\mathrm{g}}}^{\mu} \partial_{x} \alpha_{\mathrm{g}} & +P_{\mathrm{g}, v_{\mathrm{g}}}^{\mu} \partial_{x} v_{\mathrm{g}}+P_{\mathrm{g}, v_{\ell}}^{\mu} \partial_{x} v_{\ell}+P_{\mathrm{g}, \mu}^{\mu} \partial_{x} \mu \\
=\alpha_{\mathrm{g}}^{-1}\left[-\left(\Gamma_{\mathrm{g}} \Delta_{\mathrm{i}} p_{\mathrm{g}}+\rho_{\mathrm{g}} c_{\mathrm{g}}^{2}\right)\right. & \left.+\left(\xi_{\mathrm{g}}+\frac{1}{2} \Gamma_{\mathrm{g}}\left(\Delta_{\mathrm{i}} v_{\mathrm{g}}\right)^{2}\right) \mathcal{K}_{p}^{\mu}\right] I_{\mathrm{g}} \\
& +\alpha_{\mathrm{g}}^{-1}\left[\Gamma_{\mathrm{g}} \Delta_{\mathrm{i}} v_{\mathrm{g}}-\left(\xi_{\mathrm{g}}+\frac{1}{2} \Gamma_{\mathrm{g}}\left(\Delta_{\mathrm{i}} v_{\mathrm{g}}\right)^{2}\right) \mathcal{K}_{\mathrm{v}}^{\mu}\right] M_{\mathrm{g}} \\
& +\alpha_{\mathrm{g}}^{-1}\left[\Gamma_{\mathrm{g}}-\left(\xi_{\mathrm{g}}+\frac{1}{2} \Gamma_{\mathrm{g}}\left(\Delta_{\mathrm{i}} v_{\mathrm{g}}\right)^{2}\right)\left(\psi_{\mathrm{g}}+\psi_{\ell}\right) \kappa_{\mu}^{-1}\right] H_{\mathrm{g}}
\end{aligned}
$$

wherein we have defined the coefficients

$$
\begin{aligned}
P_{\mathrm{g}, \alpha_{\mathrm{g}}}^{\mu} & =\alpha_{\mathrm{g}}^{-1}\left[\left(\xi_{\mathrm{g}}+\frac{1}{2} \Gamma_{\mathrm{g}}\left(\Delta_{\mathrm{i}} v_{\mathrm{g}}\right)^{2}\right) \mathcal{A}^{\mu}-\left(\Gamma_{\mathrm{g}} \Delta_{\mathrm{i}} p_{\mathrm{g}}+\rho_{\mathrm{g}} c_{\mathrm{g}}^{2}\right) \Delta_{\mathrm{i}} v_{\mathrm{g}}\right] \\
P_{\mathrm{g}, v_{\mathrm{g}}}^{\mu} & =\rho_{\mathrm{g}} c_{\mathrm{g}}^{2}-\left(\xi_{\mathrm{g}}+\frac{1}{2} \Gamma_{\mathrm{g}}\left(\Delta_{\mathrm{i}} v_{\mathrm{g}}\right)^{2}\right) \xi_{\mathrm{g}} \alpha_{\mathrm{g}}^{-1} \kappa_{\mu}^{-1}, \\
P_{\mathrm{g}, v_{\ell}}^{\mu} & =\left(\xi_{\mathrm{g}}+\frac{1}{2} \Gamma_{\mathrm{g}}\left(\Delta_{\mathrm{i}} v_{\mathrm{g}}\right)^{2}\right) \xi_{\ell} \alpha_{\mathrm{g}}^{-1} \kappa_{\mu}^{-1}, \\
P_{\mathrm{g}, \mu}^{\mu} & =-\left(\xi_{\mathrm{g}}+\frac{1}{2} \Gamma_{\mathrm{g}}\left(\Delta_{\mathrm{i}} v_{\mathrm{g}}\right)^{2}\right)\left(v_{\mathrm{g}}-v_{\ell}\right) \alpha_{\mathrm{g}}^{-1} \kappa_{\mu}^{-1} .
\end{aligned}
$$

The corresponding expressions related to the evolution of $p_{\ell}$ are found by phase symmetry.

\subsection{Wave velocities}

We now wish to derive the wave velocities of the $\mu$-model in the homogeneous limit, where $\mathscr{I}, \mathscr{H}, \mathscr{M} \rightarrow$ 0 . In this limit, the volume fraction $\alpha_{\mathrm{g}}$ is a characteristic variable with the associated eigenvalue $v_{\mathrm{i}}$. The remaining, reduced model, i.e. (93), (95) and (96) for both phases, may then be expressed in the quasi-linear form $\partial_{t} \tilde{\mathbf{u}}_{\mu}+\tilde{\mathbf{A}}_{\mu}\left(\tilde{\mathbf{u}}_{\mu}\right) \partial_{x} \tilde{\mathbf{u}}_{\mu}=0$, where the reduced vector of unknowns is $\tilde{\mathbf{u}}_{\mu}=\left[\mu, v_{\mathrm{g}}, v_{\ell}, p_{\mathrm{g}}, p_{\ell}\right]$, and the reduced Jacobian reads

$$
\tilde{\mathbf{A}}_{\mu}=\left[\begin{array}{ccccc}
\phi_{\mathrm{g}} v_{\mathrm{g}}+\phi_{\ell} v_{\ell} & \phi_{\mathrm{g}} \xi_{\mathrm{g}} & \phi_{\ell} \xi_{\ell} & 0 & 0 \\
-\frac{\Delta_{\mathrm{i}} v_{\mathrm{g}}\left(v_{\mathrm{g}}-v_{\ell}\right)}{m_{\mathrm{g}} \kappa_{\mu}} & v_{\mathrm{g}}-\frac{\xi_{\mathrm{g}} \Delta_{\mathrm{i}} v_{\mathrm{g}}}{m_{\mathrm{g}} \kappa_{\mu}} & \frac{\xi_{\ell} \Delta_{\mathrm{i}} v_{\mathrm{g}}}{m_{\mathrm{g}} \kappa_{\mu}} & \rho_{\mathrm{g}}^{-1} & 0 \\
\frac{\Delta_{\mathrm{i}} v_{\ell}\left(v_{\mathrm{g}}-v_{\ell}\right)}{m_{\ell} \kappa_{\mu}} & \frac{\xi_{\mathrm{g}} \Delta_{\mathrm{i}} v_{\ell}}{m_{\ell} \kappa_{\mu}} & v_{\ell}-\frac{\xi_{\ell} \Delta_{\mathrm{i}} v_{\ell}}{m_{\ell} \kappa_{\mu}} & 0 & \rho_{\ell}^{-1} \\
P_{\mathrm{g}, \mu}^{\mu} & P_{\mathrm{g}, v_{\mathrm{g}}}^{\mu} & P_{\mathrm{g}, v_{\ell}}^{\mu} & v_{\mathrm{g}} & 0 \\
P_{\ell, \mu}^{\mu} & P_{\ell, v_{\mathrm{g}}}^{\mu} & P_{\ell, v_{\ell}}^{\mu} & 0 & v_{\ell}
\end{array}\right] .
$$

Again the eigenvalues $\lambda$ are given the roots of a fifth degree polynomial, for which in general no closed-form solution exists. We therefore expand in the small parameter $\varepsilon=v_{\mathrm{g}}-v_{\ell}$, i.e. $\tilde{\mathbf{A}}_{\mu}=\tilde{\mathbf{A}}_{\mu}^{(0)}+\varepsilon \tilde{\mathbf{A}}_{\mu}^{(1)}+\ldots$, and $\lambda=\lambda^{(0)}+\varepsilon \lambda^{(1)}+\ldots$ Herein, the lowest-order system matrix reads, taking $\bar{v}=\phi_{\mathrm{g}} v_{\mathrm{g}}+\phi_{\ell} v_{\ell}$,

$$
\tilde{\mathbf{A}}_{\mu}^{(0)}=\left[\begin{array}{ccccc}
\bar{v} & \phi_{\mathrm{g}} \xi_{\mathrm{g}} & \phi_{\ell} \xi_{\ell} & 0 & 0 \\
0 & \bar{v} & 0 & \rho_{\mathrm{g}}^{-1} & 0 \\
0 & 0 & \bar{v} & 0 & \rho_{\ell}^{-1} \\
0 & \rho_{\mathrm{g}} c_{\mathrm{g}}^{2}-\xi_{\mathrm{g}}^{2} /\left(\alpha_{\mathrm{g}} \kappa_{\mu}^{(0)}\right) & \xi_{\mathrm{g}} \xi_{\ell} /\left(\alpha_{\mathrm{g}} \kappa_{\mu}^{(0)}\right) & \bar{v} & 0 \\
0 & \xi_{\mathrm{g}} \xi_{\ell} /\left(\alpha_{\ell} \kappa_{\mu}^{(0)}\right) & \rho_{\ell} c_{\ell}^{2}-\xi_{\ell}^{2} /\left(\alpha_{\ell} \kappa_{\mu}^{(0)}\right) & 0 & \bar{v}
\end{array}\right]
$$


where we have used the lowest-order term of $\kappa_{\mu}$, as defined in (88):

$$
\kappa_{\mu}^{(0)}=\frac{T_{\mathrm{g}} s_{\mathrm{g}}^{2}}{\tilde{C}_{p, \mathrm{~g}}}+\frac{T_{\ell} s_{\ell}^{2}}{\tilde{C}_{p, \ell}}+\frac{\xi_{\mathrm{g}}^{2}}{m_{\mathrm{g}} c_{\mathrm{g}}^{2}}+\frac{\xi_{\ell}^{2}}{m_{\ell} c_{\ell}^{2}} .
$$

To the lowest order in $\varepsilon, v_{\mathrm{g}}=v_{\ell}=\bar{v}=v$, and thus the eigenvalue problem consists in finding the roots of $\operatorname{det}\left(\tilde{\mathbf{A}}_{\mu}^{(0)}-\lambda^{(0)} \mathbf{I}\right)=0$. Hence, the full vector of eigenvalues is given by

$$
\boldsymbol{\lambda}_{\mu}^{(0)}=\left\{v-c_{\mu,+}, v-c_{\mu,-}, v, v, v+c_{\mu,-}, v+c_{\mu,+}\right\}
$$

where the two sound speeds in the $\mu$-model are given by

$$
c_{\mu, \pm}^{2}=\frac{\left(\frac{T_{\mathrm{g}} s_{\mathrm{g}}^{2}}{\tilde{C}_{p, \mathrm{~g}}}+\frac{T_{\ell} s_{\ell}^{2}}{\tilde{C}_{p, \ell}}\right)\left(c_{\mathrm{g}}^{2}+c_{\ell}^{2}\right)+\frac{\xi_{\ell}^{2} c_{\mathrm{g}}^{2}}{m_{\ell} c_{\ell}^{2}}+\frac{\xi_{\mathrm{g}}^{2} c_{\ell}^{2}}{m_{\mathrm{g}} c_{\mathrm{g}}^{2}} \pm \sqrt{\left[\left(\frac{T_{\mathrm{g}} s_{\mathrm{g}}^{2}}{\tilde{C}_{p, \mathrm{~g}}}+\frac{T_{\ell} s_{\ell}^{2}}{\tilde{C}_{p, \ell}}\right)\left(c_{\mathrm{g}}^{2}-c_{\ell}^{2}\right)+\frac{\xi_{\ell}^{2} c_{\mathrm{g}}^{2}}{m_{\ell} c_{\ell}^{2}}-\frac{\xi_{\mathrm{g}}^{2} c_{\ell}^{2}}{m_{\mathrm{g}} c_{\mathrm{g}}^{2}}\right]^{2}+4 \frac{\xi_{\mathrm{g}}^{2} \xi_{\ell}^{2}}{m_{\mathrm{g}} m_{\ell}}}}{2\left[\frac{T_{\mathrm{g}} s_{\mathrm{g}}^{2}}{\tilde{C}_{p, \mathrm{~g}}}+\frac{T_{\tilde{C}_{\ell} s_{\ell}^{2}}}{\tilde{C}_{p, \ell}}+\frac{\xi_{\mathrm{g}}^{2}}{m_{\mathrm{g}} c_{\mathrm{g}}^{2}}+\frac{\xi_{\ell}^{2}}{m_{\ell} c_{\ell}^{2}}\right]}
$$

Proposition 6.2. The $\mu$-model satisfies the weak subcharacteristic condition with respect to the basic model of Section 2, given only the physically fundamental conditions $\rho_{k}, C_{p, k}, T_{k}>0$ for $k \in\{\mathrm{g}, \ell\}$, in the equilibrium state defined by (57).

Proof. We first note that $c_{\mu, \pm}^{2} \in \mathbb{R}$ on the given conditions, and that $c_{\mu,+}^{2} \geq 0$. The product of the sound speeds may be written as

$$
c_{\mu,+}^{-2} c_{\mu,-}^{-2}=c_{0,+}^{-2} c_{0,-}^{-2}+\mathcal{Z}_{\mu}^{0}, \quad \text { where } \quad \mathcal{Z}_{\mu}^{0}=c_{\mathrm{g}}^{-2} c_{\ell}^{-2} \frac{\frac{\xi_{\mathrm{g}}^{2}}{m_{\mathrm{g}} c_{\mathrm{g}}^{2}}+\frac{\xi_{\ell}^{2}}{m_{\ell} c_{\ell}^{2}}}{\frac{T_{\mathrm{g}} s_{\mathrm{g}}^{2}}{\tilde{C}_{p, \mathrm{~g}}}+\frac{T_{\ell} s_{\ell}^{2}}{\tilde{C}_{p, \ell}}} .
$$

Given the conditions we have that $\mathcal{Z}_{\mu}^{0} \geq 0$, and hence

$$
0 \leq c_{\mu,+}^{2} c_{\mu,-}^{2} \leq c_{0,+}^{2} c_{0,-}^{2} .
$$

Therefore also $c_{0,-}^{2}$ is positive, and thus we have that $c_{0, \pm}$ are real and, by choice, positive.

Now, the product of the differences of the sound speeds may be written as

$$
\left(c_{0,+}^{2}-c_{\mu,+}^{2}\right)\left(c_{0,+}^{2}-c_{\mu,-}^{2}\right)\left(c_{0,-}^{2}-c_{\mu,+}^{2}\right)\left(c_{0,-}^{2}-c_{\mu,-}^{2}\right)=-\mathcal{Q}_{\mu}^{0},
$$

where

$$
\mathcal{Q}_{\mu}^{0}=\left(c_{\mathrm{g}}^{2}-c_{\ell}^{2}\right)^{2} \frac{\xi_{\mathrm{g}}^{2} \xi_{\ell}^{2}}{m_{\mathrm{g}} m_{\ell}}\left[\frac{T_{\mathrm{g}} s_{\mathrm{g}}^{2}}{\tilde{C}_{p, \mathrm{~g}}}+\frac{T_{\ell} s_{\ell}^{2}}{\tilde{C}_{p, \ell}}+\frac{\xi_{\mathrm{g}}^{2}}{m_{\mathrm{g}} c_{\mathrm{g}}^{2}}+\frac{\xi_{\ell}^{2}}{m_{\ell} c_{\ell}^{2}}\right]^{-2} .
$$

Clearly, with the given conditions, $\mathcal{Q}_{\mu}^{0} \geq 0$, and hence the only ordering of sound speeds compatible with (107) and (108) is $0 \leq c_{\mu,-} \leq c_{0,-} \leq c_{\mu,+} \leq c_{0,+}$, which means that the weak subcharacteristic condition is satisfied.

Proposition 6.3. The vp-model satisfies the subcharacteristic condition with respect to the $\mu$-model, subject only to the physically fundamental conditions $\rho_{k}, C_{p, k}, T_{k}>0$, for $k \in\{\mathrm{g}, \ell\}$.

Proof. The sound speed in the $v \mu$-model is given by [42]

$$
c_{v \mu}^{2}=\frac{1}{\rho} \frac{m_{\mathrm{g}} c_{\mathrm{g}}^{2} m_{\ell} c_{\ell}^{2}\left(\frac{\xi_{\mathrm{g}}}{m_{\mathrm{g}} c_{\mathrm{g}}^{2}}+\frac{\xi_{\ell}}{m_{\ell} c_{\ell}^{2}}\right)^{2}+\left(\frac{T_{\mathrm{g}} s_{\mathrm{g}}^{2}}{\tilde{C}_{p, \mathrm{~g}}}+\frac{T_{\ell} s_{\ell}^{2}}{\tilde{C}_{p, \ell}}\right)\left(m_{\mathrm{g}} c_{\mathrm{g}}^{2}+m_{\ell} c_{\ell}^{2}\right)}{\frac{T_{\mathrm{g}} s_{\mathrm{g}}^{2}}{\tilde{C}_{p, \mathrm{~g}}}+\frac{T_{\ell} s_{\ell}^{2}}{\tilde{C}_{p, \ell}}+\frac{\xi_{\mathrm{g}}^{2}}{m_{\mathrm{g}} c_{\mathrm{g}}^{2}}+\frac{\xi_{\ell}^{2}}{m_{\ell} c_{\ell}^{2}}} .
$$


We now consider the product of the differences in the sound speeds of the two models, which may be written as

$$
\left(c_{\mu,+}^{2}-c_{v \mu}^{2}\right)\left(c_{\mu,-}^{2}-c_{v \mu}^{2}\right)=-\mathcal{Q}_{v \mu}^{\mu},
$$

where

$$
\mathcal{Q}_{v \mu}^{\mu}=Y_{\mathrm{g}} Y_{\ell}\left[\frac{\left(\frac{T_{\mathrm{g}} s_{\mathrm{g}}^{2}}{m_{\mathrm{g}} C_{p, \mathrm{~g}}}+\frac{T_{\ell} s_{\ell}^{2}}{m_{\ell} C_{p, \ell}}\right)\left(c_{\mathrm{g}}^{2}-c_{\ell}^{2}\right)-\frac{\xi_{\mathrm{g}}^{2} c_{\ell}^{2}}{m_{\mathrm{g}} c_{\mathrm{g}}^{2}}+\frac{\xi_{\ell} c_{\mathrm{g}}^{2}}{m_{\ell} c_{\ell}^{2}}+\left(\frac{1}{m_{\mathrm{g}}}-\frac{1}{m_{\ell}}\right) \xi_{\mathrm{g}} \xi_{\ell}}{\frac{T_{\mathrm{g}} s_{\mathrm{g}}^{2}}{\tilde{C}_{p, \mathrm{~g}}}+\frac{T_{\ell} s_{\ell}^{2}}{\tilde{C}_{p, \ell}}+\frac{\xi_{\mathrm{g}}^{2}}{m_{\mathrm{g}} c_{\mathrm{g}}^{2}}+\frac{\xi_{\ell}^{2}}{m_{\ell} c_{\ell}^{2}}}\right]^{2} .
$$

Clearly $\mathcal{Q}_{v \mu}^{\mu} \geq 0$. Hence, exactly one of the factors on the left hand side of (111) must be negative, which gives $c_{\mu,-} \leq c_{v \mu} \leq c_{\mu,+}$, i.e. the subcharacteristic condition is satisfied.

\section{THE $p \mu$-MODEL}

We now consider the model which arises when we impose instantaneous mechanical-chemical equilibrium, i.e. we let the relaxation parameters $\mathscr{I}, \mathscr{K} \rightarrow \infty$, which we expect corresponds to

$$
p_{\mathrm{g}}=p_{\ell} \equiv p \quad \text { and } \quad \mu_{\mathrm{g}}=\mu_{\ell} \equiv \mu .
$$

Simultaneously, $I_{\mathrm{g}}=\mathscr{I}\left(p_{\mathrm{g}}-p_{\ell}\right)$ and $K_{\mathrm{g}}=\mathscr{K}\left(\mu_{\ell}-\mu_{\mathrm{g}}\right)$ should remain finite. We now seek explicit expressions for these terms in order to find the governing equations of the model.

In the following analysis we use the parameter set stated in Section 2 and therefore let the interfacial pressure jump $\Delta_{\mathrm{i}} p=p_{\mathrm{i}}-p=0$. From (35) and (85) we have

$$
\begin{aligned}
\mathrm{D}_{k} p & =-\frac{\rho_{k} c_{k}^{2}}{\alpha_{k}}\left(\tilde{I}_{k}+\partial_{x} \alpha_{k} v_{k}\right)+\frac{\xi_{k}+\frac{1}{2} \Gamma_{k}\left(\Delta_{\mathrm{i}} v_{k}\right)^{2}}{\alpha_{k}} K_{k}+\frac{\Gamma_{k}}{\alpha_{k}} \Delta_{\mathrm{i}} v_{k} M_{k}+\frac{\Gamma_{k}}{\alpha_{k}} H_{k}, \\
\mathrm{D}_{k} \mu & =-\frac{\xi_{k}}{\alpha_{k}}\left(\tilde{I}_{k}+\partial_{x} \alpha_{k} v_{k}\right)+\left[\frac{\xi_{k}^{2}}{m_{k} c_{k}^{2}}+\frac{T_{k} s_{k}^{2}}{\tilde{C}_{p, k}}+\frac{1}{2}\left(\Delta_{\mathrm{i}} v_{k}\right)^{2} \psi_{k}\right] K_{k}+\psi_{k} \Delta_{\mathrm{i}} v_{k} M_{k}+\psi_{k} H_{k} .
\end{aligned}
$$

where we have defined $\tilde{I}_{k}=I_{k}-v_{\mathrm{i}} \partial_{x} \alpha_{k}=\partial_{t} \alpha_{k}$.

Eqs. (114) and (115) evaluated for each phase now constitute a $4 \times 4$ system which is straightforward to solve for the four unknowns $\partial p / \partial t, \partial \mu / \partial t, \tilde{I}_{\mathrm{g}}$, and $K_{\mathrm{g}}$, in terms of spatial derivatives and the remaining source terms. The final expressions for the latter two are

$$
\begin{gathered}
\tilde{I}_{\mathrm{g}}=-\mathcal{P}_{p}^{p \mu}\left(v_{\mathrm{g}}-v_{\ell}\right) \partial_{x} p-\mathcal{G}_{p}^{p \mu}\left(v_{\mathrm{g}}-v_{\ell}\right) \partial_{x} \mu-\Phi_{\mathrm{g}} \partial_{x} \alpha_{\mathrm{g}} v_{\mathrm{g}}+\Phi_{\ell} \partial_{x} \alpha_{\ell} v_{\ell}+I_{v}^{p \mu} M_{\mathrm{g}}+I_{T}^{p \mu} H_{\mathrm{g}}, \\
K_{\mathrm{g}}=-\mathcal{P}_{\mu}^{p \mu}\left(v_{\mathrm{g}}-v_{\ell}\right) \partial_{x} p-\mathcal{G}_{\mu}^{p \mu}\left(v_{\mathrm{g}}-v_{\ell}\right) \partial_{x} \mu-\mathcal{V}_{\mu, \mathrm{g}}^{p \mu} \partial_{x} \bar{v}+\mathcal{K}_{v}^{p \mu} M_{\mathrm{g}}+\mathcal{K}_{T}^{p \mu} H_{\mathrm{g}},
\end{gathered}
$$

where the coefficients are given in Appendix A.

\subsection{Governing equations}

Inserting the expressions (116) and (117) into the basic model of Section 2, we are now in a position to state the full model. The mechanical-chemical equilibrium model may thus be formulated as follows.

- Conservation of mass: $\partial_{t} \rho+\partial_{x}\left(m_{\mathrm{g}} v_{\mathrm{g}}+m_{\ell} v_{\ell}\right)=0$,

- Momentum balance:

$$
\begin{aligned}
& \partial_{t} m_{\mathrm{g}} v_{\mathrm{g}}+\partial_{x} m_{\mathrm{g}} v_{\mathrm{g}}^{2}+\left(\alpha_{\mathrm{g}}+v_{\mathrm{i}} \Phi_{\mu}^{p \mu}\left(v_{\mathrm{g}}-v_{\ell}\right)\right) \partial_{x} p+v_{\mathrm{i}} \mathcal{G}_{\mu}^{p \mu}\left(v_{\mathrm{g}}-v_{\ell}\right) \partial_{x} \mu+v_{\mathrm{i}} \mathcal{V}_{\mu, \mathrm{g}}^{p \mu} \partial_{x} \bar{v} \\
& =\left(1+v_{\mathrm{i}} \mathcal{K}_{v}^{p \mu}\right) M_{\mathrm{g}}+v_{\mathrm{i}} \mathcal{K}_{T}^{p \mu} H_{\mathrm{g}},
\end{aligned}
$$


- Energy balance:

$$
\begin{aligned}
\partial_{t} E_{\mathrm{g}}+\partial_{x} E_{\mathrm{g}} v_{\mathrm{g}}+\left[\alpha_{\mathrm{g}} v_{\mathrm{g}}+\left(\left(\mu+\frac{1}{2} v_{\mathrm{i}}^{2}\right) \mathcal{P}_{\mu}^{p \mu}-p \mathcal{P}_{p}^{p \mu}\right)\left(v_{\mathrm{g}}-v_{\ell}\right)\right] \partial_{x} p & \\
& +\left[\left(\mu+\frac{1}{2} v_{\mathrm{i}}^{2}\right) \mathcal{G}_{\mu}^{p \mu}-p \mathcal{G}_{p}^{p \mu}\right]\left(v_{\mathrm{g}}-v_{\ell}\right) \partial_{x} \mu+\left[\left(\mu+\frac{1}{2} v_{\mathrm{i}}^{2}\right) \mathcal{V}_{\mu, \mathrm{g}}^{p \mu}+p \Phi_{\ell}\right] \partial_{x} \bar{v} \\
& =\left[v_{\mathrm{i}}+\left(\mu+\frac{1}{2} v_{\mathrm{i}}^{2}\right) \mathcal{K}_{v}^{p \mu}-p I_{v}^{p \mu}\right] M_{\mathrm{g}}+\left[1+\left(\mu+\frac{1}{2} v_{\mathrm{i}}^{2}\right) \mathcal{K}_{T}^{p \mu}-p I_{T}^{p \mu}\right] H_{\mathrm{g}} .
\end{aligned}
$$

The momentum and energy equations for the liquid phase are found by phase symmetry. Note that, like other $p$-relaxed models, the $p \mu$-model is expected to be non-hyperbolic for nonzero difference in the velocity unless a regularising interfacial pressure $p_{\mathrm{i}}$ is defined.

\subsection{Wave velocities}

We now wish to write the system in a quasilinear form, in order to find the wave speeds of the system, in the homogeneous limit where we let the relaxation terms $\mathscr{M}, \mathscr{H} \rightarrow 0$. To this end, we will express the model in the vector of unknowns $\mathbf{u}_{p \mu}=\left[p, \mu, \bar{v}, v_{\mathrm{g}}, v_{\ell}\right]$. We therefore seek the evolution equations for the elements of $\mathbf{u}_{p \mu}$.

For the volume evolution, we find, using (24) and (116), that

$$
\partial_{t} \alpha_{\mathrm{g}}+\mathcal{P}_{p}^{p \mu}\left(v_{\mathrm{g}}-v_{\ell}\right) \partial_{x} p+\mathcal{G}_{p}^{p \mu}\left(v_{\mathrm{g}}-v_{\ell}\right) \partial_{x} \mu+\Phi_{\mathrm{g}} \partial_{x} \alpha_{\mathrm{g}} v_{\mathrm{g}}-\Phi_{\ell} \partial_{x} \alpha_{\ell} v_{\ell}=0,
$$

For the volume-averaged velocity $\bar{v}$ we find, using (25), (116), (117) and (120), that

$$
\begin{array}{r}
\partial_{t} \bar{v}+\left(\alpha_{\mathrm{g}} \rho_{\mathrm{g}}^{-1}+\alpha_{\ell} \rho_{\ell}^{-1}+P_{\bar{v}}^{p \mu} \varepsilon^{2}\right) \partial_{x} p+G_{\bar{v}}^{p \mu} \varepsilon^{2} \partial_{x} \mu+\alpha_{\mathrm{g}} \varepsilon \partial_{x} v_{\mathrm{g}} \\
\quad-\alpha_{\ell} \varepsilon \partial_{x} v_{\ell}+\left(\Phi_{\mathrm{g}} v_{\mathrm{g}}+\Phi_{\ell} v_{\ell}-V_{\bar{v}, \mathrm{~g}}^{p \mu} \varepsilon\right) \partial_{x} \bar{v}=0
\end{array}
$$

where we have defined the shorthand coefficients $P_{\bar{v}}^{p \mu}, G_{\bar{v}}^{p \mu}, V_{\bar{v}, \mathrm{~g}}^{p \mu}$ (for which expressions are given in Appendix A), used $\varepsilon=v_{\mathrm{g}}-v_{\ell}$, and inserted $\beta_{\mathrm{g}}=1-\beta_{\ell}=\sqrt{T_{\ell}} /\left(\sqrt{T_{\mathrm{g}}}+\sqrt{T_{\ell}}\right)$. Now, for the pressure and chemical potentials, we get from (114) and (115) that

$$
\begin{aligned}
& \partial_{t} p+\left(\Psi_{\mathrm{g}}^{p} v_{\mathrm{g}}+\Psi_{\ell}^{p} v_{\ell}\right) \partial_{x} p+G_{p}^{p \mu} \varepsilon \partial_{x} \mu+V_{p}^{p \mu} \partial_{x} \bar{v}=0 \\
& \partial_{t} \mu+P_{\mu}^{p \mu} \varepsilon \partial_{x} p+\left(\Psi_{\mathrm{g}}^{\mu} v_{\mathrm{g}}+\Psi_{\ell}^{\mu} v_{\ell}\right) \partial_{x} \mu+V_{\mu}^{p \mu} \partial_{x} \bar{v}=0 .
\end{aligned}
$$

Again, the coefficients are given in Appendix A.

The homogeneous system in a quasilinear form thus reads $\partial_{t} \mathbf{u}_{p \mu}+\mathbf{A}_{p \mu}\left(\mathbf{u}_{p \mu}\right) \partial_{x} \mathbf{u}_{p \mu}=0$, where the system Jacobian is given by

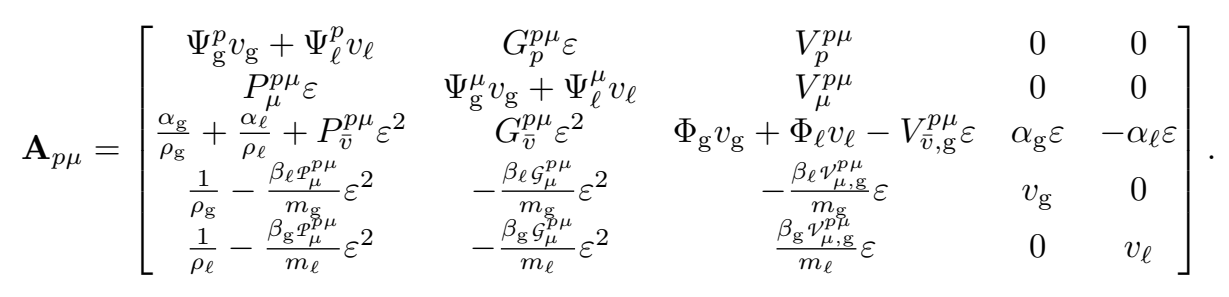

Obtaining the assocated eigenvalues exactly by analytic means is again unfeasible, as the problem consists in finding the roots of a fifth-degree polynomial. We therefore expand in $\varepsilon: \mathbf{A}_{p \mu}=\mathbf{A}_{p \mu}^{(0)}+\varepsilon \mathbf{A}_{p \mu}^{(1)}+\varepsilon^{2} \mathbf{A}_{p \mu}^{(2)}+\ldots$, where it is assumed that the matrices $\mathbf{A}_{p \mu}^{(i)}$ are independent of $\varepsilon$. To the lowest order, where $\varepsilon \rightarrow 0$, taking 
$v=v_{\mathrm{g}}=v_{\ell}$, we get the matrix

$$
\mathbf{A}_{p \mu}^{(0)}=\left[\begin{array}{ccccc}
v & 0 & V_{p}^{p \mu,(0)} & 0 & 0 \\
0 & v & V_{\mu}^{p \mu,(0)} & 0 & 0 \\
\frac{\alpha_{\mathrm{g}}}{\rho_{\mathrm{g}}}+\frac{\alpha_{\ell}}{\rho_{\ell}} & 0 & v & 0 & 0 \\
\frac{1}{\rho_{\mathrm{g}}} & 0 & 0 & v & 0 \\
\frac{1}{\rho_{\ell}} & 0 & 0 & 0 & v
\end{array}\right]
$$

where the superscript "(0)" on the coefficients signifies the zeroth-order expansion in $\varepsilon$, such that

$$
V_{p}^{p \mu,(0)}=\frac{\frac{T_{\mathrm{g}} s_{\mathrm{g}}^{2}}{\tilde{C}_{p, \mathrm{~g}}}+\frac{T_{\ell} s_{\ell}^{2}}{\tilde{C}_{p, \ell}}}{\left(\frac{\alpha_{\mathrm{g}}}{\rho_{\mathrm{g}} c_{\mathrm{g}}^{2}}+\frac{\alpha_{\ell}}{\rho_{\ell} c_{\ell}^{2}}\right)\left(\frac{T_{\mathrm{g}} s_{\mathrm{g}}^{2}}{\tilde{C}_{p, \mathrm{~g}}}+\frac{T_{\ell} s_{\ell}^{2}}{\tilde{C}_{p, \ell}}\right)+\left(\frac{\xi_{\mathrm{g}}}{\rho_{\mathrm{g}} c_{\mathrm{g}}^{2}}-\frac{\xi_{\ell}}{\rho_{\ell} c_{\ell}^{2}}\right)^{2}} .
$$

The eigenvalues in the $p \mu$-model are, to the lowest order in $\varepsilon$,

$$
\boldsymbol{\lambda}_{p \mu}^{(0)}=\left\{v-c_{p \mu}, v, v, v, v+c_{p \mu}\right\},
$$

where we have identified the sound speed $c_{p \mu}$ of the model, given by

$$
c_{p \mu}^{2}=\frac{\left(\frac{\alpha_{\mathrm{g}}}{\rho_{\mathrm{g}}}+\frac{\alpha_{\ell}}{\rho_{\ell}}\right)\left(\frac{T_{\mathrm{g}} s_{\mathrm{g}}^{2}}{\tilde{C}_{p, \mathrm{~g}}}+\frac{T_{\ell} s_{\ell}^{2}}{\tilde{C}_{p, \ell}}\right)}{\left(\frac{\alpha_{\mathrm{g}}}{\rho_{\mathrm{g}} c_{\mathrm{g}}^{2}}+\frac{\alpha_{\ell}}{\rho_{\ell} c_{\ell}^{2}}\right)\left(\frac{T_{\mathrm{g}} s_{\mathrm{g}}^{2}}{\tilde{C}_{p, \mathrm{~g}}}+\frac{T_{2} s_{\ell}^{2}}{\tilde{C}_{p, \ell}}\right)+\left(\frac{\xi_{\mathrm{g}}}{\rho_{\mathrm{g}} c_{\mathrm{g}}^{2}}-\frac{\xi_{\ell}}{\rho_{\ell} c_{\ell}^{2}}\right)^{2}} .
$$

Proposition 7.1. The pu-model satisfies the weak subcharacteristic condition with respect to the p-model, given only the physically fundamental conditions $\rho_{k}, C_{p, k}, T_{k}>0$, for $k \in\{\mathrm{g}, \ell\}$, in the equilibrium state defined by (57).

Proof. From (62) and (128), we observe that we may write

$$
c_{p \mu}^{-2}=c_{p}^{-2}+\mathcal{Z}_{p \mu}^{p}, \quad \text { where } \quad \mathcal{Z}_{p \mu}^{p}=\frac{\left(\frac{\xi_{\mathrm{g}}}{\rho_{\mathrm{g}} c_{\mathrm{g}}^{2}}-\frac{\xi_{\ell}}{\rho_{\ell} c_{\ell}^{2}}\right)^{2}}{\left(\frac{\alpha_{\mathrm{g}}}{\rho_{\mathrm{g}}}+\frac{\alpha_{\ell}}{\rho_{\ell}}\right)\left(\frac{T_{\mathrm{g}} s_{\mathrm{g}}^{2}}{\tilde{C}_{p, \mathrm{~g}}}+\frac{T_{\ell} s_{\ell}^{2}}{\tilde{C}_{p, \ell}}\right)} .
$$

Due to the given physical conditions, $\mathcal{Z}_{p \mu}^{p} \geq 0$, and hence $0 \leq c_{p \mu} \leq c_{p}$, i.e. the weak subcharacteristic condition is satisfied.

Proposition 7.2. The pu-model satisfies the weak subcharacteristic condition with respect to the $\mu$-model, under the physically fundamental conditions $\rho_{k}, C_{p, k}, T_{k}>0$, for $k \in\{\mathrm{g}, \ell\}$, in the equilibrium state defined by (57).

Proof. Using the expressions (105) and (128) for the sound speeds in the two models, we may write

$$
\left(c_{\mu,+}^{2}-c_{p \mu}^{2}\right)\left(c_{\mu,-}^{2}-c_{p \mu}^{2}\right)=-\mathcal{Q}_{p \mu}^{\mu},
$$

where

$$
\mathcal{Q}_{p \mu}^{\mu}=\frac{\frac{\alpha_{\mathrm{g}} \alpha_{\ell}}{\rho_{\mathrm{g}} c_{\mathrm{g}} \rho_{\ell} c_{\ell}^{2}}\left(\frac{T_{\mathrm{g}} s_{\mathrm{g}}^{2}}{\tilde{C}_{p, \mathrm{~g}}}+\frac{T_{\ell} s_{\ell}^{2}}{\tilde{C}_{p, \ell}}\right)\left[\left(\frac{T_{\mathrm{g}} s_{\mathrm{g}}^{2}}{\tilde{C}_{p, \mathrm{~g}}}+\frac{T_{\ell} s_{\ell}^{2}}{\tilde{C}_{p, \ell}}\right)\left(c_{\mathrm{g}}^{2}-c_{\ell}^{2}\right)-\left(\frac{\xi_{\mathrm{g}}}{\rho_{\mathrm{g}} c_{\mathrm{g}}^{2}}-\frac{\xi_{\ell}}{\rho_{\ell} c_{\ell}^{2}}\right)\left(\frac{\xi_{\mathrm{g}} c_{\ell}^{2}}{\alpha_{\mathrm{g}}}+\frac{\xi_{\ell} c_{\mathrm{g}}^{2}}{\alpha_{\ell}}\right)\right]^{2}}{\left[\frac{T_{\mathrm{g}} s_{\mathrm{g}}^{2}}{\tilde{C}_{p, \mathrm{~g}}}+\frac{T_{\ell} s_{\ell}^{2}}{\tilde{C}_{p, \ell}}+\frac{\xi_{\mathrm{g}}^{2}}{m_{\mathrm{g}} c_{\mathrm{g}}^{2}}+\frac{\xi_{\ell}^{2}}{m_{\ell} c_{\ell}^{2}}\right]\left[\left(\frac{\alpha_{\mathrm{g}}}{\rho_{\mathrm{g}} c_{\mathrm{g}}^{2}}+\frac{\alpha_{\ell}}{\rho_{\ell} c_{\ell}^{2}}\right)\left(\frac{T_{\mathrm{g}} s_{\mathrm{g}}^{2}}{\tilde{C}_{p, \mathrm{~g}}}+\frac{T_{\ell} s_{\ell}^{2}}{\tilde{C}_{p, \ell}}\right)+\left(\frac{\xi_{\mathrm{g}}}{\rho_{\mathrm{g}} c_{\mathrm{g}}^{2}}-\frac{\xi_{\ell}}{\rho_{\ell} c_{\ell}^{2}}\right)^{2}\right]^{2}} .
$$

Clearly, on the given conditions, $\mathcal{Q}_{p \mu}^{\mu} \geq 0$. Therefore, exactly one factor on the left hand side of (130) is negative, and hence $c_{\mu,-} \leq c_{p \mu} \leq c_{\mu,+}$, so the weak subcharacteristic condition is satisfied. 


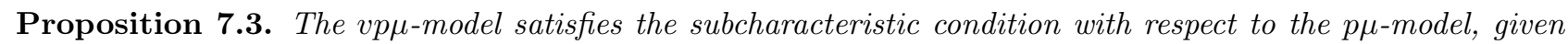
the physically fundamental conditions $\rho_{k}, C_{p, k}, T_{k}>0$.

Proof. The sound speed in the $v p \mu$-model is given by $[22,42]$

$$
c_{v p \mu}^{2}=\frac{1}{\rho} \frac{\frac{T_{\mathrm{g}} s_{\mathrm{g}}^{2}}{\tilde{C}_{p, \mathrm{~g}}}+\frac{T_{\ell} s_{\ell}^{2}}{\tilde{C}_{p, \ell}}}{\left(\frac{\alpha_{\mathrm{g}}}{\rho_{\mathrm{g}} c_{\mathrm{g}}^{2}}+\frac{\alpha_{\ell}}{\rho_{\ell} c_{\ell}^{2}}\right)\left(\frac{T_{\mathrm{g}} s_{\mathrm{g}}^{2}}{\tilde{C}_{p, \mathrm{~g}}}+\frac{T_{\ell} s_{\ell}^{2}}{\tilde{C}_{p, \ell}}\right)+\left(\frac{\xi_{\mathrm{g}}}{\rho_{\mathrm{g}} c_{\mathrm{g}}^{2}}-\frac{\xi_{\ell}}{\rho_{\ell} c_{\ell}^{2}}\right)^{2}} .
$$

Now, we may write

$$
c_{v p \mu}^{-2}=c_{p \mu}^{-2}+\mathcal{Z}_{v p \mu}^{p \mu}, \quad \text { where } \quad \mathcal{Z}_{v p \mu}^{p \mu}=\frac{\alpha_{\mathrm{g}} \alpha_{\ell}}{\rho_{\mathrm{g}} \rho_{\ell}}\left(\rho_{\ell}-\rho_{\mathrm{g}}\right)^{2} c_{p \mu}^{-2},
$$

which is clearly positive, due to the given conditions. Thus, $0 \leq c_{v p \mu} \leq c_{p \mu}$, i.e. the subcharacteristic condition is satisfied.

Remark 7.4. By direct comparison of (128) and (132), we find the ratio

$$
\frac{c_{p \mu}}{c_{v p \mu}}=\sqrt{\rho\left(\frac{\alpha_{\mathrm{g}}}{\rho_{\mathrm{g}}}+\frac{\alpha_{\ell}}{\rho_{\ell}}\right)}
$$

This is exactly the same ratio as has been shown to hold for other models associated with $v$-relaxation in the $p$-branch of the hierarchy $[21,46]$. We can thus extend the relation

$$
\frac{c_{p}}{c_{v p}}=\frac{c_{p T}}{c_{v p T}}=\frac{c_{p T \mu}}{c_{v p T \mu}}=\frac{c_{p \mu}}{c_{v p \mu}}
$$

by the newly obtained ratio (134) between the sound speeds of the $v p \mu$ - and $p \mu$-models.

Proposition 7.5. The $p T \mu$-model satisfies the weak subcharacteristic condition with respect to the pu-model, given the physically fundamental conditions $\rho_{k}, C_{p, k}, T>0$, in the equilibrium state defined by (57).

Proof. In the equilibrium state defined by the $p T \mu$-model, we have $T_{\mathrm{g}}=T_{\ell} \equiv T$. The sound velocity in the $p T \mu$-model is given in [46], and may be rewritten as

$$
c_{p T \mu}^{2}=\frac{\frac{\alpha_{\mathrm{g}}}{\rho_{\mathrm{g}}}+\frac{\alpha_{\ell}}{\rho_{\ell}}}{\frac{\alpha_{\mathrm{g}}}{\rho_{\mathrm{g}} c_{\mathrm{g}}^{2}}+\frac{\alpha_{\ell}}{\rho_{\ell} c_{\ell}^{2}}+\tilde{C}_{p, \mathrm{~g}} T\left[\frac{1}{\Delta h}\left(\frac{1}{\rho_{\ell}}-\frac{1}{\rho_{\mathrm{g}}}\right)+\frac{\Gamma_{\mathrm{g}}}{\rho_{\mathrm{g}} c_{\mathrm{g}}^{2}}\right]^{2}+\tilde{C}_{p, \ell} T\left[\frac{1}{\Delta h}\left(\frac{1}{\rho_{\mathrm{g}}}-\frac{1}{\rho_{\ell}}\right)-\frac{\Gamma_{\ell}}{\rho_{\ell} c_{\ell}^{2}}\right]^{2}},
$$

where we have introduced the enthalpy difference $\Delta h=h_{\mathrm{g}}-h_{\ell}$.

We may reorganize the last equality in (135) to yield

$$
\frac{c_{p \mu}}{c_{p T \mu}}=\frac{c_{v p \mu}}{c_{v p T \mu}}
$$

Flåtten and Lund [22] showed that the subcharacteristic condition is satisfied between the models on the right hand side, i.e. that $0 \leq c_{v p T \mu} \leq c_{v p \mu}$. The same must hold for the models on the left hand side of (137), i.e. $0 \leq c_{p T \mu} \leq c_{p \mu}$, and hence the weak subcharacteristic condition is satisfied. In particular, we may write the sound speed as

$$
c_{p T \mu}^{-2}=c_{p \mu}^{-2}+\mathcal{Z}_{p T \mu}^{p \mu},
$$


where

$$
\mathcal{Z}_{p T \mu}^{p \mu}=\tilde{C}_{p, \mathrm{~g}} \tilde{C}_{p, \ell} T \frac{\left[\frac{1}{\Delta h}\left(\frac{1}{\rho_{\ell}}-\frac{1}{\rho_{\mathrm{g}}}\right)\left(\frac{s_{\mathrm{g}}}{\tilde{C}_{p, \mathrm{~g}}}+\frac{s_{\ell}}{\tilde{C}_{p, \ell}}\right)+\frac{\Gamma_{\mathrm{g}}}{\rho_{\mathrm{g}} c_{\mathrm{g}}^{2}} \frac{s_{\ell}}{\tilde{C}_{p, \ell}}+\frac{\Gamma_{\ell}}{\rho_{\ell} c_{\ell}^{2}} \frac{s_{\mathrm{g}}}{\tilde{C}_{p, \mathrm{~g}}}\right]^{2}}{\left(\frac{\alpha_{\mathrm{g}}}{\rho_{\mathrm{g}}}+\frac{\alpha_{\ell}}{\rho_{\ell}}\right)\left(\frac{s_{\mathrm{g}}^{2}}{\tilde{C}_{p, \mathrm{~g}}}+\frac{s_{\ell}^{2}}{\tilde{C}_{p, \ell}}\right)} .
$$

Clearly, $\mathcal{Z}_{p T \mu}^{p \mu} \geq 0$ based on the given conditions.

\section{THe $T \mu$-MODEL}

We now investigate the model which arises when we assume instantaneous thermal-chemical equilibrium, i.e. let the relaxation parameters $\mathscr{K}, \mathscr{H} \rightarrow \infty$, which expectedly corresponds to

$$
T_{\mathrm{g}}=T_{\ell} \equiv T \quad \text { and } \quad \mu_{\mathrm{g}}=\mu_{\ell} \equiv \mu .
$$

The products $H_{\mathrm{g}}=\mathscr{H}\left(T_{\ell}-T_{\mathrm{g}}\right)$ and $K_{\mathrm{g}}=\mathscr{K}\left(\mu_{\ell}-\mu_{\mathrm{g}}\right)$ remain finite, and may be expressed in terms of spatial derivatives and remaining source terms. In the forthcoming, we seek explicit expressions for these terms to insert into the basic model of Section 2.

The equilibrium conditions are contained in (68) and (85). These may be combined to yield

$$
K_{\mathrm{g}}=-\mathcal{A}_{\mu}^{T \mu} \partial_{x} \alpha_{\mathrm{g}}-\mathcal{G}_{\mu}^{T \mu} \varepsilon \partial_{x} \mu-\mathcal{T}_{\mu}^{T \mu} \varepsilon \partial_{x} T-\mathcal{V}_{\mu, \mathrm{g}}^{T \mu} \partial_{x} \alpha_{\mathrm{g}} v_{\mathrm{g}}+\mathcal{V}_{\mu, \ell}^{T \mu} \partial_{x} \alpha_{\ell} v_{\ell}+\mathcal{K}_{p}^{T \mu} \tilde{I}_{\mathrm{g}}+\mathcal{K}_{\mathrm{v}}^{T \mu} \varepsilon M_{\mathrm{g}}
$$

where the coefficients are given in Appendix B.

\subsection{Governing equations}

We are now in a position to state the $T \mu$-model in its entirety, by inserting (141) into the basic model of Section 2. The model can be expressed by the following equation set:

- Volume advection: $\partial_{t} \alpha_{\mathrm{g}}+v_{\mathrm{i}} \partial_{x} \alpha_{\mathrm{g}}=I_{\mathrm{g}}$,

- Conservation of mass: $\partial_{t} \rho+\partial_{x}\left(\alpha_{\mathrm{g}} \rho_{\mathrm{g}} v_{\mathrm{g}}+\alpha_{\ell} \rho_{\ell} v_{\ell}\right)=0$,

- Conservation of momentum:

$$
\begin{aligned}
& \partial_{t} \alpha_{\mathrm{g}} \rho_{\mathrm{g}} v_{\mathrm{g}}+\partial_{x}\left(\alpha_{\mathrm{g}} \rho_{\mathrm{g}} v_{\mathrm{g}}^{2}+\alpha_{\mathrm{g}} p_{\mathrm{g}}\right) \\
&+v_{\mathrm{i}}\left[\mathcal{G}_{\mu}^{T \mu}\left(v_{\mathrm{g}}-v_{\ell}\right) \partial_{x} \mu+\mathcal{T}_{\mu}^{T \mu}\left(v_{\mathrm{g}}-v_{\ell}\right) \partial_{x} T+v_{\mu, \mathrm{g}}^{T \mu} \partial_{x} \alpha_{\mathrm{g}} v_{\mathrm{g}}-\mathcal{V}_{\mu, \ell}^{T \mu} \partial_{x} \alpha_{\ell} v_{\ell}\right] \\
& \quad+\left(v_{\mathrm{i}}^{2}\left(v_{\mu, \mathrm{g}}^{T \mu}+\mathcal{V}_{\mu, \ell}^{T \mu}\right)-p_{\mathrm{i}}\right) \partial_{x} \alpha_{\mathrm{g}}=v_{\mathrm{i}} \mathcal{K}_{p}^{T \mu} I_{\mathrm{g}}+\left(1+v_{\mathrm{i}} \mathcal{K}_{v}^{T \mu}\left(v_{\mathrm{g}}-v_{\ell}\right)\right) M_{\mathrm{g}}
\end{aligned}
$$

- Conservation of energy: $\partial_{t} E+\partial_{x}\left(E_{\mathrm{g}} v_{\mathrm{g}}+E_{\ell} v_{\ell}+\alpha_{\mathrm{g}} v_{\mathrm{g}} p_{\mathrm{g}}+\alpha_{\ell} v_{\ell} p_{\ell}\right)=0$.

\subsection{Wave velocities}

We now seek the wave velocities of the model in the homogeneous limit, where $\mathscr{I}, \mathscr{M} \rightarrow 0$. As usual, we are interested in the zeroth-order expansion in $\varepsilon=v_{\mathrm{g}}-v_{\ell} \cdot{ }^{3}$ We may therefore directly evaluate the evolution equations in this limit, and take $v_{\mathrm{g}}=v_{\ell}=v$ if they are outside the differential operator.

After some tedious, but fairly straightforward algebra, we find that to the lowest order in $\varepsilon$, the wave velocities of the $T \mu$-model are given by

$$
\boldsymbol{\lambda}_{T \mu}^{(0)}=\left\{v-c_{T \mu,+}, v-c_{T \mu,-}, v, v+c_{T \mu,-}, v+c_{T \mu,+}\right\} .
$$

\footnotetext{
${ }^{3}$ Strictly speaking, exact eigenvalues may be found analytically in this model, since noting that $\alpha_{\mathrm{g}}$ is a characteristic variable reduces the eigenvalue problem to finding the solutions of a fourth-degree polynomial, which is analytically tractable.
} 
Herein, $c_{T \mu, \pm}$ are the sound speeds of this model, which may be expressed by

$$
\begin{aligned}
& c_{T \mu, \pm}^{2}= \frac{1}{2}\left\{\frac{\Delta h^{2} m_{\mathrm{g}} m_{\ell}\left(c_{\mathrm{g}}^{2}+c_{\ell}^{2}\right)}{\tilde{C}_{p, \mathrm{~g}} \tilde{C}_{p, \ell} T_{\mathrm{g}}^{2} c_{\ell}^{2}}+\frac{m_{\ell}+m_{\mathrm{g}}\left(1+\frac{\Gamma_{\ell}}{c_{\ell}^{2}} \Delta h\right)^{2}}{\tilde{C}_{p, \mathrm{~g}} T}+\frac{m_{\mathrm{g}}+m_{\ell}\left(1-\frac{\Gamma_{\mathrm{g}}}{c_{\mathrm{g}}^{2}} \Delta h\right)^{2}}{\tilde{C}_{p, \ell} T} \pm\right. \\
& {\left.\left[\left(\frac{\Delta h^{2} m_{\mathrm{g}} m_{\ell}\left(c_{\mathrm{g}}^{2}-c_{\ell}^{2}\right)}{\tilde{C}_{p, \mathrm{~g}} \tilde{C}_{p, \ell} T^{2} c_{\mathrm{g}}^{2} c_{\ell}^{2}}-\frac{m_{\ell}-m_{\mathrm{g}}\left(1+\frac{\Gamma_{\ell}}{c_{\ell}^{2}} \Delta h\right)^{2}}{\tilde{C}_{p, \mathrm{~g}} T}+\frac{m_{\mathrm{g}}-m_{\ell}\left(1-\frac{\Gamma_{\mathrm{g}}}{c_{\mathrm{g}}^{2}} \Delta h\right)^{2}}{\tilde{C}_{p, \ell} T}\right)^{2}+4 m_{\mathrm{g}} m_{\ell}\left(\frac{1+\frac{\Gamma_{\ell}}{c_{\ell}^{2}} \Delta h}{\tilde{C}_{p, \mathrm{~g}} T}+\frac{1-\frac{\Gamma_{\mathrm{g}}}{c_{\mathrm{g}}^{2}} \Delta h}{\tilde{C}_{p, \ell} T}\right)^{2}\right]^{\frac{1}{2}}\right\} } \\
& \times\left[\frac{\Delta h^{2} m_{\mathrm{g}} m_{\ell}}{\tilde{C}_{p, \mathrm{~g}} \tilde{C}_{p, \ell} T^{2} c_{\mathrm{g}}^{2} c_{\ell}^{2}}+\frac{\frac{m_{\ell}}{c_{\ell}^{2}}+\frac{m_{\mathrm{g}}}{c_{\mathrm{g}}^{2}}\left(1+\frac{\Gamma_{\ell}}{c_{\ell}^{2}} \Delta h\right)^{2}}{\tilde{C}_{p, \mathrm{~g}} T}+\frac{\frac{m_{\mathrm{g}}}{c_{\mathrm{g}}^{2}}+\frac{m_{\ell}}{c_{\ell}^{2}}\left(1-\frac{\Gamma_{\mathrm{g}}}{c_{\mathrm{g}}^{2}} \Delta h\right)^{2}}{\tilde{C}_{p, \ell} T}+\left(\frac{\Gamma_{\ell}}{c_{\ell}^{2}}-\frac{\Gamma_{\mathrm{g}}}{c_{\mathrm{g}}^{2}}-\frac{\Gamma_{\mathrm{g}} \Gamma_{\ell}}{c_{\mathrm{g}}^{2} c_{\ell}^{2}} \Delta h\right)^{2}\right]^{-1} .
\end{aligned}
$$

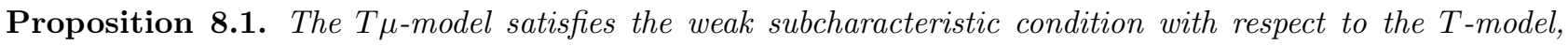
given the physically fundamental conditions $\rho_{k}, C_{p, k}, T>0$, in the equilibrium state defined by (57).

Proof. We may write

$$
\left(c_{T,+}^{2}-c_{T \mu,+}^{2}\right)\left(c_{T,+}^{2}-c_{T \mu,-}^{2}\right)\left(c_{T,-}^{2}-c_{T \mu,+}^{2}\right)\left(c_{T,-}^{2}-c_{T \mu,-}^{2}\right)=-\mathcal{Q}_{T \mu}^{T},
$$

where

$$
\begin{aligned}
\mathcal{Q}_{T \mu}^{T}=m_{\mathrm{g}} m_{\ell} & {\left[\left(\frac{1}{\tilde{C}_{p, \ell} T}+\frac{1+\Gamma_{\ell} c_{\ell}^{-2} \Delta h}{\tilde{C}_{p, \mathrm{~g}} T}\right)\left(\frac{1}{\tilde{C}_{p, \mathrm{~g}} T}+\frac{1-\Gamma_{\mathrm{g}} c_{\mathrm{g}}^{-2} \Delta h}{\tilde{C}_{p, \ell} T}+\frac{\Gamma_{\ell}}{m_{\ell}}\left(\frac{\Gamma_{\ell}}{c_{\ell}^{2}}-\frac{\Gamma_{\mathrm{g}}}{c_{\mathrm{g}}^{2}}-\frac{\Gamma_{\mathrm{g}} \Gamma_{\ell}}{c_{\mathrm{g}}^{2} c_{\ell}^{2}} \Delta h\right)\right) c_{\mathrm{g}}^{2}\right.} \\
& \left.-\left(\frac{1}{\tilde{C}_{p, \mathrm{~g}} T}+\frac{1-\Gamma_{\mathrm{g}} c_{\mathrm{g}}^{-2} \Delta h}{\tilde{C}_{p, \ell} T}\right)\left(\frac{1}{\tilde{C}_{p, \ell} T}+\frac{1+\Gamma_{\ell} c_{\ell}^{-2} \Delta h}{\tilde{C}_{p, \mathrm{~g}} T}-\frac{\Gamma_{\mathrm{g}}}{m_{\mathrm{g}}}\left(\frac{\Gamma_{\ell}}{c_{\ell}^{2}}-\frac{\Gamma_{\mathrm{g}}}{c_{\mathrm{g}}^{2}}-\frac{\Gamma_{\mathrm{g}} \Gamma_{\ell}}{c_{\mathrm{g}}^{2} c_{\ell}^{2}} \Delta h\right)\right) c_{\ell}^{2}\right]^{2} \\
\times & {\left[\left(\frac{\Delta h^{2} m_{\mathrm{g}} m_{\ell}}{\tilde{C}_{p, \mathrm{~g}} \tilde{C}_{p, \ell} T^{2} c_{\mathrm{g}}^{2} c_{\ell}^{2}}+\frac{1}{\tilde{C}_{p, \mathrm{~g}} T}\left(\frac{m_{\ell}}{c_{\ell}^{2}}+\frac{m_{\mathrm{g}}}{c_{\mathrm{g}}^{2}}\left(1+\frac{\Gamma_{\ell}}{c_{\ell}^{2}} \Delta h\right)^{2}\right)+\frac{1}{\tilde{C}_{p, \ell} T}\left(\frac{m_{\mathrm{g}}}{c_{\mathrm{g}}^{2}}+\frac{m_{\ell}}{c_{\ell}^{2}}\left(1-\frac{\Gamma_{\mathrm{g}}}{c_{\mathrm{g}}^{2}} \Delta h\right)^{2}\right)\right.\right.} \\
& \left.\left.+\left(\frac{\Gamma_{\ell}}{c_{\ell}^{2}}-\frac{\Gamma_{\mathrm{g}}}{c_{\mathrm{g}}^{2}}-\frac{\Gamma_{\mathrm{g}} \Gamma_{\ell}}{c_{\mathrm{g}}^{2} c_{\ell}^{2}} \Delta h\right)^{2}\right)\left(\frac{1}{\tilde{C}_{p, \mathrm{~g}} T}+\frac{1}{\tilde{C}_{p, \ell} T}+\frac{\Gamma_{\ell}^{2}}{m_{\ell} c_{\ell}^{2}}+\frac{\Gamma_{\mathrm{g}}^{2}}{m_{\mathrm{g}} c_{\mathrm{g}}^{2}}\right)\right]^{-2} .
\end{aligned}
$$

Moreover, we may write

$$
\left(c_{T,+}^{2}+c_{T,-}^{2}\right)-\left(c_{T \mu,+}^{2}+c_{T \mu,-}^{2}\right)=\mathcal{Z}_{T \mu}^{T}
$$

where

$$
\begin{aligned}
\mathcal{Z}_{T \mu}^{T}=\left[\left(\frac{1}{\tilde{C}_{p, \mathrm{~g}} T}+\frac{1-\frac{\Gamma_{\mathrm{g}}}{c_{\mathrm{g}}^{2}} \Delta h}{\tilde{C}_{p, \ell} T}+\frac{\Gamma_{\ell}}{m_{\ell}}\left(\frac{\Gamma_{\ell}}{c_{\ell}^{2}}-\frac{\Gamma_{\mathrm{g}}}{c_{\mathrm{g}}^{2}}-\frac{\Gamma_{\mathrm{g}} \Gamma_{\ell}}{c_{\mathrm{g}}^{2} c_{\ell}^{2}} \Delta h\right)\right) \frac{m_{\ell} c_{\mathrm{g}}^{2}}{c_{\ell}^{2}}\right. \\
\left.+\left(\frac{1}{\tilde{C}_{p, \ell} T}+\frac{1+\frac{\Gamma_{\ell}}{c_{\ell}^{2}} \Delta h}{\tilde{C}_{p, \mathrm{~g}} T}-\frac{\Gamma_{\mathrm{g}}}{m_{\mathrm{g}}}\left(\frac{\Gamma_{\ell}}{c_{\ell}^{2}}-\frac{\Gamma_{\mathrm{g}}}{c_{\mathrm{g}}^{2}}-\frac{\Gamma_{\mathrm{g}} \Gamma_{\ell}}{c_{\mathrm{g}}^{2} c_{\ell}^{2}} \Delta h\right)\right) \frac{m_{\mathrm{g}} c_{\ell}^{2}}{c_{\mathrm{g}}^{2}}\right] \\
\times\left[\left(\frac{\Delta h^{2} m_{\mathrm{g}} m_{\ell}}{\tilde{C}_{p, \mathrm{~g}} \tilde{C}_{p, \ell} T^{2} c_{\mathrm{g}}^{2} c_{\ell}^{2}}+\frac{\frac{m_{\ell}}{c_{\ell}^{2}}+\frac{m_{\mathrm{g}}}{c_{\mathrm{g}}^{2}}\left(1+\frac{\Gamma_{\ell}}{c_{\ell}^{2}} \Delta h\right)^{2}}{\tilde{C}_{p, \mathrm{~g}} T}+\frac{\frac{m_{\mathrm{g}}}{c_{\mathrm{g}}^{2}}+\frac{m_{\ell}}{c_{\ell}^{2}}\left(1-\frac{\Gamma_{\mathrm{g}}}{c_{\mathrm{g}}^{2}} \Delta h\right)^{2}}{\tilde{C}_{p, \ell} T}+\left(\frac{\Gamma_{\ell}}{c_{\ell}^{2}}-\frac{\Gamma_{\mathrm{g}}}{c_{\mathrm{g}}^{2}}-\frac{\Gamma_{\mathrm{g}} \Gamma_{\ell}}{c_{\mathrm{g}}^{2} c_{\ell}^{2}} \Delta h\right)^{2}\right)\right. \\
\left.\times\left(\frac{1}{\tilde{C}_{p, \mathrm{~g}} T}+\frac{1}{\tilde{C}_{p, \ell} T}+\frac{\Gamma_{\ell}^{2}}{m_{\ell} c_{\ell}^{2}}+\frac{\Gamma_{\mathrm{g}}^{2}}{m_{\mathrm{g}} c_{\mathrm{g}}^{2}}\right)\right]^{-1} .
\end{aligned}
$$


Clearly, $\mathcal{Z}_{T \mu}^{T} \geq 0$ and $\mathcal{Q}_{T \mu}^{T} \geq 0$ on grounds of the given conditions. The only possible ordering of sound speeds is thus $0 \leq c_{T \mu,-} \leq c_{T,-} \leq c_{T \mu,+} \leq c_{T,+}$, i.e. the weak subcharacteristic condition is satisfied.

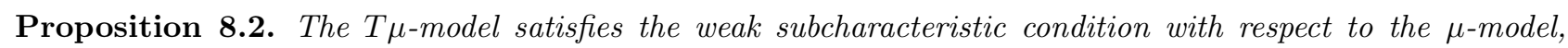
given the physically fundamental conditions $\rho_{k}, C_{p, k}, T>0$, in the equilibrium state defined by (57).

Proof. We note that we may write

$$
\left(c_{\mu,+}^{2}-c_{T \mu,+}^{2}\right)\left(c_{\mu,+}^{2}-c_{T \mu,-}^{2}\right)\left(c_{\mu,-}^{2}-c_{T \mu,+}^{2}\right)\left(c_{\mu,-}^{2}-c_{T \mu,-}^{2}\right)=-\mathcal{Q}_{T \mu}^{\mu},
$$

where

$$
\begin{aligned}
& \mathcal{Q}_{T \mu}^{\mu}=m_{\mathrm{g}} m_{\ell} {\left[\left(\frac{s_{\ell}}{\tilde{C}_{p, \ell}}+\frac{s_{\mathrm{g}}\left(1+\frac{\Gamma_{\ell}}{c_{\ell}^{2}} \Delta h\right)}{\tilde{C}_{p, \mathrm{~g}}}\right)\left(\frac{s_{\mathrm{g}}}{\tilde{C}_{p, \mathrm{~g}}}+\frac{s_{\ell}\left(1-\frac{\Gamma_{\mathrm{g}}}{c_{\mathrm{g}}^{2}} \Delta h\right)}{\tilde{C}_{p, \ell}}-\left(\frac{\Gamma_{\ell}}{c_{\ell}^{2}}-\frac{\Gamma_{\mathrm{g}}}{c_{\mathrm{g}}^{2}}-\frac{\Gamma_{\mathrm{g}} \Gamma_{\ell}}{c_{\mathrm{g}}^{2} c_{\ell}^{2}} \Delta h\right) \frac{\xi_{\ell}}{m_{\ell}}\right) c_{\mathrm{g}}^{2}\right.} \\
&\left.-\left(\frac{s_{\mathrm{g}}}{\tilde{C}_{p, \mathrm{~g}}}+\frac{s_{\ell}\left(1-\frac{\Gamma_{\mathrm{g}}}{c_{\mathrm{g}}^{2}} \Delta h\right)}{\tilde{C}_{p, \ell}}\right)\left(\frac{s_{\ell}}{\tilde{C}_{p, \ell}}+\frac{s_{\mathrm{g}}\left(1+\frac{\Gamma_{\ell}}{c_{\ell}^{2}} \Delta h\right)}{\tilde{C}_{p, \mathrm{~g}}}+\left(\frac{\Gamma_{\ell}}{c_{\ell}^{2}}-\frac{\Gamma_{\mathrm{g}}}{c_{\mathrm{g}}^{2}}-\frac{\Gamma_{\mathrm{g}} \Gamma_{\ell}}{c_{\mathrm{g}}^{2} c_{\ell}^{2}} \Delta h\right) \frac{\xi_{\mathrm{g}}}{m_{\mathrm{g}}}\right) c_{\ell}^{2}\right]^{2} \\
& \times {\left[\left(\frac{\Delta h^{2} m_{\mathrm{g}} m_{\ell}}{\tilde{C}_{p, \mathrm{~g}} \tilde{C}_{p, \ell} T^{2} c_{\mathrm{g}}^{2} c_{\ell}^{2}}+\frac{1}{\tilde{C}_{p, \mathrm{~g}} T}\left(\frac{m_{\ell}}{c_{\ell}^{2}}+\frac{m_{\mathrm{g}}}{c_{\mathrm{g}}^{2}}\left(1+\frac{\Gamma_{\ell}}{c_{\ell}^{2}} \Delta h\right)^{2}\right)+\frac{1}{\tilde{C}_{p, \ell} T}\left(\frac{m_{\mathrm{g}}}{c_{\mathrm{g}}^{2}}+\frac{m_{\ell}}{c_{\ell}^{2}}\left(1-\frac{\Gamma_{\mathrm{g}}}{c_{\mathrm{g}}^{2}} \Delta h\right)^{2}\right)\right.\right.} \\
&\left.\left.+\left(\frac{\Gamma_{\ell}}{c_{\ell}^{2}}-\frac{\Gamma_{\mathrm{g}}}{c_{\mathrm{g}}^{2}}-\frac{\Gamma_{\mathrm{g}} \Gamma_{\ell}}{c_{\mathrm{g}}^{2} c_{\ell}^{2}} \Delta h\right)^{2}\right)\left(\frac{T s_{\mathrm{g}}^{2}}{\tilde{C}_{p, \mathrm{~g}}}+\frac{T s_{\ell}^{2}}{\tilde{C}_{p, \ell}}+\frac{\xi_{\mathrm{g}}^{2}}{m_{\mathrm{g}} c_{\mathrm{g}}^{2}}+\frac{\xi_{\ell}^{2}}{m_{\ell} c_{\ell}^{2}}\right)\right]^{-2} .
\end{aligned}
$$

Now, we may also write $\left(c_{\mu,+}^{2}+c_{\mu,-}^{2}\right)-\left(c_{T \mu,+}^{2}+c_{T \mu,-}^{2}\right)=\mathcal{Z}_{T \mu}^{\mu}$, where

$$
\begin{gathered}
\mathcal{Z}_{T \mu}^{\mu}=\left\{\left[\frac{s_{\mathrm{g}}}{\tilde{C}_{p, \mathrm{~g}}}+\frac{s_{\ell}\left(1-\frac{\Gamma_{\mathrm{g}}}{c_{\mathrm{g}}^{2}} \Delta h\right)}{\tilde{C}_{p, \ell}}-\left(\frac{\Gamma_{\ell}}{c_{\ell}^{2}}-\frac{\Gamma_{\mathrm{g}}}{c_{\mathrm{g}}^{2}}-\frac{\Gamma_{\mathrm{g}} \Gamma_{\ell}}{c_{\mathrm{g}}^{2} c_{\ell}^{2}} \Delta h\right) \frac{\xi_{\ell}}{m_{\ell}}\right]^{2} \frac{m_{\ell} c_{\mathrm{g}}^{2}}{c_{\ell}^{2}}\right. \\
\left.+\left[\frac{s \ell}{\tilde{C}_{p, \ell}}+\frac{s_{\mathrm{g}}\left(1+\frac{\Gamma_{\ell}}{c_{\ell}^{2}} \Delta h\right)}{\tilde{C}_{p, \mathrm{~g}}}+\left(\frac{\Gamma_{\ell}}{c_{\ell}^{2}}-\frac{\Gamma_{\mathrm{g}}}{c_{\mathrm{g}}^{2}}-\frac{\Gamma_{\mathrm{g}} \Gamma_{\ell}}{c_{\mathrm{g}}^{2} c_{\ell}^{2}} \Delta h\right) \frac{\xi_{\mathrm{g}}}{m_{\mathrm{g}}}\right]^{2} \frac{m_{\mathrm{g}} c_{\ell}^{2}}{c_{\mathrm{g}}^{2}}\right\} \\
\times\left[\left(\frac{\Delta h^{2} m_{\mathrm{g}} m_{\ell}}{\tilde{C}_{p, \mathrm{~g}} \tilde{C}_{p, \ell} T^{2} c_{\mathrm{g}}^{2} c_{\ell}^{2}}+\frac{1}{\tilde{C}_{p, \mathrm{~g}} T}\left(\frac{m_{\ell}}{c_{\ell}^{2}}+\frac{m_{\mathrm{g}}}{c_{\mathrm{g}}^{2}}\left(1+\frac{\Gamma_{\ell}}{c_{\ell}^{2}} \Delta h\right)^{2}\right)\right.\right. \\
\left.\left.+\frac{1}{\tilde{C}_{p, \ell} T}\left(\frac{m_{\mathrm{g}}}{c_{\mathrm{g}}^{2}}+\frac{m_{\ell}}{c_{\ell}^{2}}\left(1-\frac{\Gamma_{\mathrm{g}}}{c_{\mathrm{g}}^{2}} \Delta h\right)^{2}\right)+\left(\frac{\Gamma_{\ell}}{c_{\ell}^{2}}-\frac{\Gamma_{\mathrm{g}}}{c_{\mathrm{g}}^{2}}-\frac{\Gamma_{\mathrm{g}} \Gamma_{\ell}}{c_{\mathrm{g}}^{2} c_{\ell}^{2}} \Delta h\right)^{2}\right)\left(\frac{T s_{\mathrm{g}}^{2}}{\tilde{C}_{p, \mathrm{~g}}}+\frac{T s_{\ell}^{2}}{\tilde{C}_{p, \ell}}+\frac{\xi_{\mathrm{g}}^{2}}{m_{\mathrm{g}} c_{\mathrm{g}}^{2}}+\frac{\xi_{\ell}^{2}}{m_{\ell} c_{\ell}^{2}}\right)\right]^{-1} .
\end{gathered}
$$

Clearly, $\mathcal{Q}_{T \mu}^{\mu} \geq 0$ and $\mathcal{Z}_{T \mu}^{\mu} \geq 0$ for the given conditions. The only possible ordering of the sound speeds is therefore $0 \leq c_{T \mu,-} \leq c_{\mu,-} \leq c_{T \mu,+} \leq c_{\mu,+}$, i.e. the eigenvalues of the relaxed model are interlaced between the eigenvalues of the parent model, and the weak subcharacteristic condition is satisfied.

Proposition 8.3. The $v T \mu$-model satisfies the subcharacteristic condition with respect to the T $\mu$-model, given the physically fundamental conditions $\rho_{k}, C_{p, k}, T>0$.

Proof. The sound speed in the $v T \mu$-model is given by [42]

$$
c_{v T \mu}^{2}=\frac{\frac{\Delta h^{2} m_{\mathrm{g}} m_{\ell} c_{v}^{2}}{\tilde{C}_{p, \mathrm{~g}} \tilde{C}_{p, \ell} T^{2} c_{\mathrm{g}} c_{\ell}^{2} \rho}+\frac{\left(\rho+m_{\mathrm{g}} \frac{\Gamma_{\ell}}{c_{\ell}^{2}} \Delta h\right)^{2}}{\frac{\tilde{C}_{p, \mathrm{~g}} T}{\Delta h^{2} m_{\mathrm{g}} m_{\ell}}}+\frac{\left(\rho-m_{\ell} \frac{\Gamma_{\mathrm{g}}}{c_{\mathrm{g}}^{2}} \Delta h\right)^{2}}{\tilde{C}_{p, \ell} T}}{\frac{\frac{m_{\ell}}{c_{\ell}^{2}}+\frac{m_{\mathrm{g}}}{c_{\mathrm{g}}^{2}}\left(1+\frac{\Gamma_{\ell}}{c_{\ell}^{2}} \Delta h\right)^{2}}{\tilde{C}_{p, \mathrm{~g}} \tilde{C}_{p, \ell} T^{2} c_{\mathrm{g}}^{2} c_{\ell}^{2}}+\frac{\frac{m_{\mathrm{g}}}{c_{\mathrm{g}}^{2}}+\frac{m_{\ell}}{c_{\ell}^{2}}\left(1-\frac{\Gamma_{\mathrm{g}}}{c_{\mathrm{g}}^{2}} \Delta h\right)^{2}}{\tilde{C}_{p, \ell} T}+\left(\frac{\Gamma_{\ell}}{c_{\ell}^{2}}-\frac{\Gamma_{\mathrm{g}}}{c_{\mathrm{g}}^{2}}-\frac{\Gamma_{\mathrm{g}} \Gamma_{\ell}}{c_{\mathrm{g}}^{2} c_{\ell}^{2}} \Delta h\right)^{2}}
$$


We may now write the product of the differences between the sound speeds as

$$
\left(c_{T \mu,+}^{2}-c_{v T \mu}^{2}\right)\left(c_{T \mu,-}^{2}-c_{v T \mu}^{2}\right)=-\mathcal{Q}_{v T \mu}^{T \mu},
$$

where

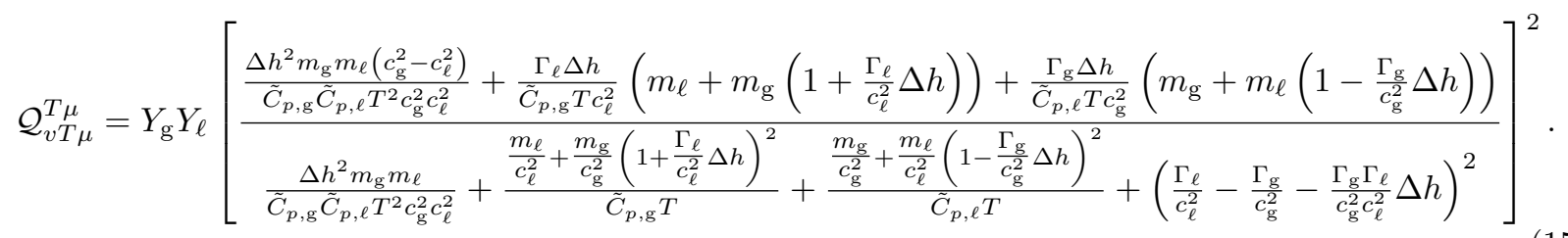

Due to the given conditions, it is clear that $\mathcal{Q}_{v T \mu}^{T \mu} \geq 0$, and thus exactly one of the factors on the left hand side of (153) is negative. Hence, the sound speeds must be ordered as $c_{T \mu,-} \leq c_{v T \mu} \leq c_{T \mu,+}$, i.e. the subcharacteristic condition is satisfied.

Proposition 8.4. The $p T \mu$-model satisfies the weak subcharacteristic condition with respect to the T $\mu$-model, subject to the physically fundamental conditions $\rho_{k}, C_{p, k}, T>0$, in the equilibrium state defined by (57).

Proof. The sound speed in the $p T \mu$-model is stated in (136). We note that we may write

$$
\left(c_{T \mu,+}^{2}-c_{p T \mu}^{2}\right)\left(c_{T \mu,-}^{2}-c_{p T \mu}^{2}\right)=-\mathcal{Q}_{p T \mu}^{T \mu},
$$

where

$$
\begin{aligned}
& \mathcal{Q}_{p T \mu}^{T \mu}=\frac{\tilde{C}_{p, g} \tilde{C}_{, \ell \ell} T^{2}}{\Delta h^{2} \rho_{\mathrm{g}}^{2} \rho_{\ell}^{2}} \\
& \times\left[\frac{\Delta h^{2} m_{\mathrm{g}} m_{\ell}}{\tilde{C}_{p, \mathrm{~g}} \tilde{C}_{p, \ell} T^{2} c_{\mathrm{g}}^{2} c_{\ell}^{2}}\left(c_{\mathrm{g}}^{2}-c_{\ell}^{2}\right)+\frac{\rho_{\mathrm{g}} \rho_{\ell}}{\tilde{C}_{p, \ell} T}\left(\frac{1}{\rho_{\ell}}-\frac{1-\frac{\Gamma_{\mathrm{g}}}{c_{\mathrm{g}}^{2}} \Delta h}{\rho_{\mathrm{g}}}\right)\left(1-\alpha_{\ell} \frac{\Gamma_{\mathrm{g}}}{c_{\mathrm{g}}^{2}} \Delta h\right)-\frac{\rho_{\mathrm{g}} \rho_{\ell}}{\tilde{C}_{p, \mathrm{~g}} T}\left(\frac{1}{\rho_{\mathrm{g}}}-\frac{1+\frac{\Gamma_{\ell}}{c_{\ell}^{2}} \Delta h}{\rho_{\ell}}\right)\left(1+\alpha_{\mathrm{g}} \frac{\Gamma_{\ell}}{c_{\ell}^{2}} \Delta h\right)\right]^{2} \\
& \times\left[\frac{\Delta h^{2} m_{\mathrm{g}} m_{\ell}}{\tilde{C}_{p, \mathrm{~g}} \tilde{C}_{p, \ell} T^{2} c_{\mathrm{g}}^{2} c_{\ell}^{2}}+\frac{\frac{m_{\ell}}{c_{\ell}^{2}}+\frac{m_{\mathrm{g}}}{c_{\mathrm{g}}^{2}}\left(1+\frac{\Gamma_{\ell}}{c_{\ell}^{2}} \Delta h\right)^{2}}{\tilde{C}_{p, \mathrm{~g}} T}+\frac{\frac{m_{\mathrm{g}}}{c_{\mathrm{g}}^{2}}+\frac{m_{\ell}}{c_{\ell}^{2}}\left(1-\frac{\Gamma_{\mathrm{g}}}{c_{\mathrm{g}}^{2}} \Delta h\right)^{2}}{\tilde{C}_{p, \ell} T}+\left(\frac{\Gamma_{\ell}}{c_{\ell}^{2}}-\frac{\Gamma_{\mathrm{g}}}{c_{\mathrm{g}}^{2}}-\frac{\Gamma_{\mathrm{g}} \Gamma_{\ell}}{c_{\mathrm{g}}^{2} c_{\ell}^{2}} \Delta h\right)^{2}\right]^{-1} \\
& \times\left[\frac{\alpha_{\mathrm{g}}}{\rho_{\mathrm{g}} c_{\mathrm{g}}^{2}}+\frac{\alpha_{\ell}}{\rho_{\ell} c_{\ell}^{2}}+\frac{\tilde{C}_{p, \mathrm{~g}} T}{\Delta h^{2}}\left(\frac{1}{\rho_{\ell}}-\frac{1-\frac{\Gamma_{\mathrm{g}}}{c_{\mathrm{g}}^{2}} \Delta h}{\rho_{\mathrm{g}}}\right)^{2}+\frac{\tilde{C}_{p, \ell} T}{\Delta h^{2}}\left(\frac{1}{\rho_{\mathrm{g}}}-\frac{1+\frac{\Gamma_{\ell}}{c_{\ell}^{2}} \Delta h}{\rho_{\ell}}\right)^{2}\right]^{-2} \cdot(156)
\end{aligned}
$$

Due to the given conditions, it is clear that $\mathcal{Q}_{p T \mu}^{T \mu} \geq 0$. Hence, exactly one of the factors on the left hand side of (155) must be negative, and therefore $c_{T \mu,-} \leq c_{p T \mu} \leq c_{T \mu,+}$, i.e. the weak subcharacteristic condition is satisfied.

\section{COMPARISON AND DISCUSSION OF MODELS}

In this section, we compare the models in the hierarchy. We first show plots for relevant cases, and then briefly discuss physical and numerical aspects of the different models.

\subsection{Comparison of sound speeds}

We now present plots of the sound speeds in all the models in the hierarchy, for different physically relevant conditions, in order to illustrate the effect of different equilibrium assumptions. Plots with the same physical parameters were presented by Lund [42] for the $v$-branch of the hierarchy, building on plots by [22]. [21] and [46] 
presented similar plots for the $p$-branch of the hierarchy. In the following, results for the complete hierarchy are shown.

The main panel of Figure 2 shows the fluid-mechanical sound speeds of all the models in the hierarchy for a water-steam mixture at atmospheric conditions. The thermophysical parameters are shown in Table 1. Apart from the fact that the subcharacteristic condition is always respected, we notice that there are mainly two equilibrium assumptions that affect the propagation speeds, namely those of $p$ - and $v$-relaxation, respectively. First, imposing instantaneous equilibrium in pressure attracts the sound velocities to the lower bound of the parent models, which can be seen in the insets of Figure 2. Further imposing velocity equilibrium, the sound velocity is reduced by a factor (see Remark 7.4)

$$
\sqrt{\rho\left(\frac{\alpha_{\mathrm{g}}}{\rho_{\mathrm{g}}}+\frac{\alpha_{\ell}}{\rho_{\ell}}\right)} \simeq \sqrt{\alpha_{\mathrm{g}} \alpha_{\ell} \frac{\rho_{\ell}}{\rho_{\mathrm{g}}}} .
$$

The approximation made is valid when $\rho_{\mathrm{g}} \ll \rho_{\ell}$, which is the case here. Hence, these equilibrium assumptions seem to have severe impact on the wave velocities, in particular when the density difference between the phases is large.

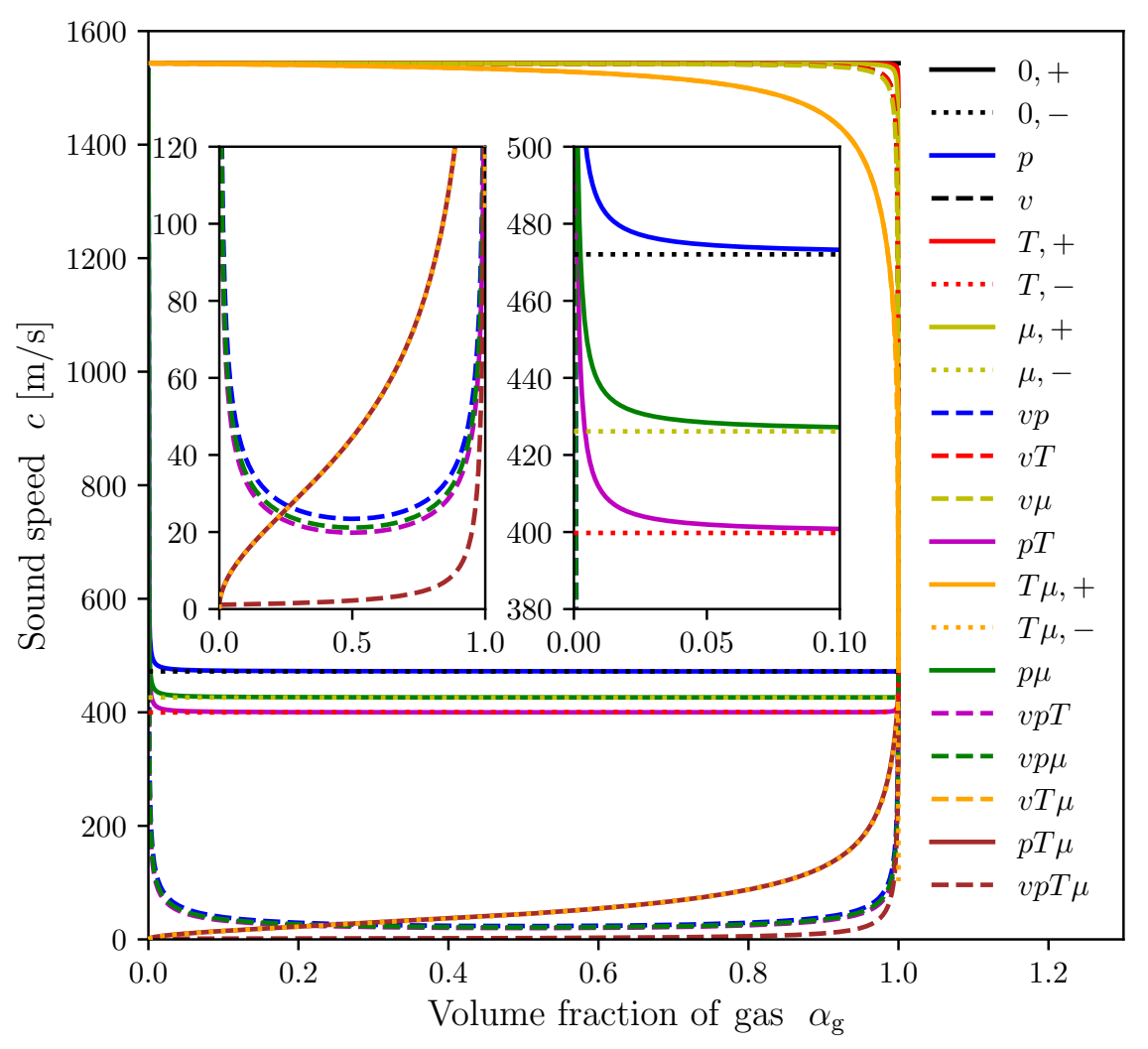

FIgURE 2. Sound velocities in a water-steam mixture at atmospheric conditions. The insets show close-ups of parts of the plots in the main panel.

In Figure 3, the sound speeds in the entire hierarchy are plotted for a $\mathrm{CO}_{2}$ mixture at 50 bar, whose thermophysical properties are given in Table 2. By close inspection, it can be seen that the subcharacteristic condition is everywhere respected. In particular, the sound speeds of an equilibrium system are always interlaced between 
TABLE 1. Parameters for a water-steam mixture at atmospheric pressure.

\begin{tabular}{lllcc}
\hline Quantity & Symbol & Unit & Gas & Liquid \\
\hline Pressure & $p$ & $\mathrm{MPa}$ & 0.1 & 0.1 \\
Temperature & $T$ & $\mathrm{~K}$ & 372.76 & 372.76 \\
Density & $\rho$ & $\mathrm{kg} \mathrm{m}^{-3}$ & 0.59031 & 958.64 \\
Speed of sound & $c$ & $\mathrm{~m} \mathrm{~s}^{-1}$ & 472.05 & 1543.4 \\
Heat capacity & $C_{p}$ & $\mathrm{~J} \mathrm{~kg}^{-1} \mathrm{~K}^{-1}$ & 2075.9 & 4216.1 \\
Entropy & $s$ & $\mathrm{~m}^{2} \mathrm{~s}^{-2} \mathrm{~K}^{-1}$ & 7358.8 & 1302.6 \\
Grüneisen coefficient & $\Gamma$ & $(-)$ & 0.33699 & 0.4 \\
\hline
\end{tabular}

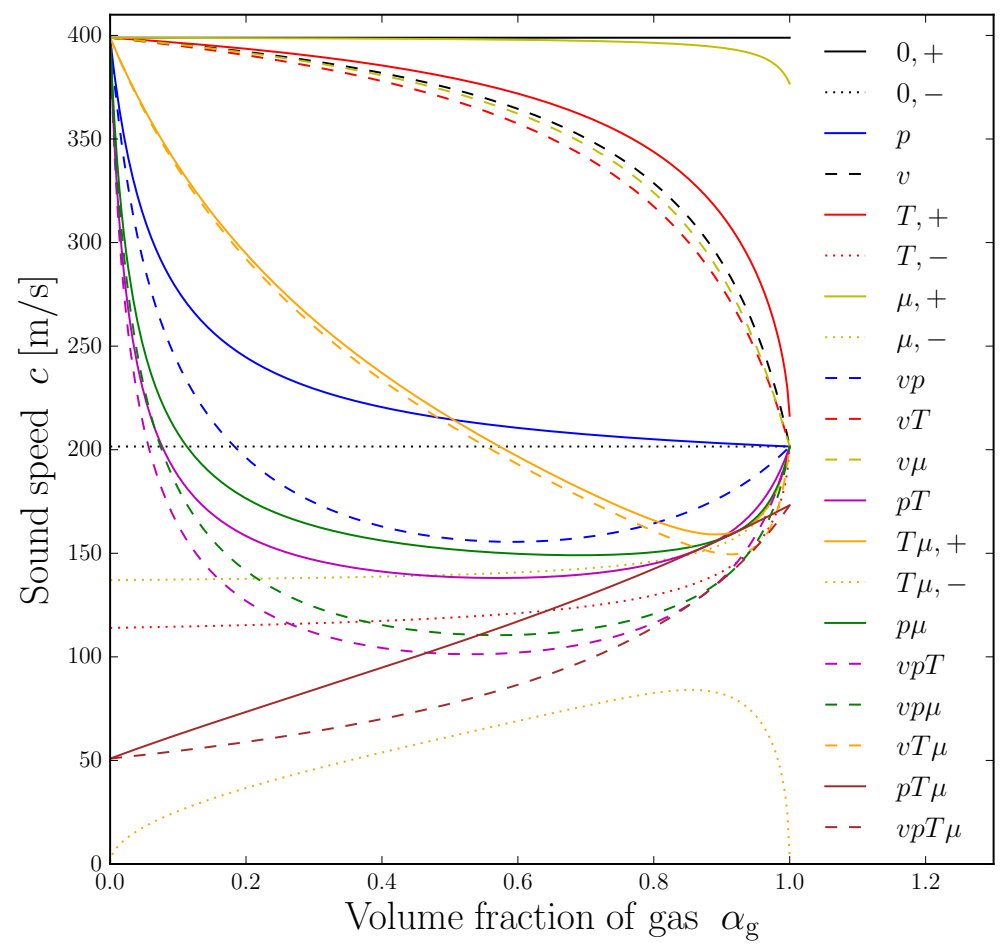

Figure 3. Sound speeds in a two-phase $\mathrm{CO}_{2}$ mixture at 50 bar.

the sound speeds in the parent models. Again, the pressure relaxation has the most prominent effect on the sound speed, but also combining thermal and chemical equilibrium seems to have a strong effect.

\subsection{Discontinuous sound speeds}

All the models considered in the present paper are only strictly valid when the gas fraction $\alpha_{\mathrm{g}} \in(0,1)$. One would expect the sound speeds of the models to be continuous at the phase boundary, i.e. at the transition between single and two-phase flow, in the sense that the two-phase speed of sound should reduce to the singlephase speed of sound in the limit where one phase disappears:

$$
\lim _{\alpha_{k} \rightarrow 1} c_{X} \rightarrow c_{k}
$$


for a given model $X$ in the hierarchy. However, some of the models have wave speeds that are discontinuous at the phase boundary. In particular, this concerns the $p T \mu$ - and $v p T \mu$-models, whose sound speeds are discontinuous in both limits $\alpha_{k} \rightarrow 1$, which can be seen directly by evaluating the analytic expressions in these limits (see Refs. [42,46]).

The $T$ - and $\mu$-models have "half-continuous" sound speeds, in the sense that for the " \pm " sound waves, only one of them is continuous in the limit $\alpha_{k} \rightarrow 1$. For the $\mu$-model, taking $\alpha_{\ell} \rightarrow 1$ in (105) yields

$$
\lim _{\alpha_{\ell} \rightarrow 1} c_{\mu, \pm}^{2}=\frac{\frac{T_{\mathrm{g}} s_{\mathrm{g}}^{2}}{C_{p, \mathrm{~g}}}\left(c_{\mathrm{g}}^{2}+c_{\ell}^{2}\right)+\frac{\xi_{\mathrm{g}}^{2} c_{\ell}^{2}}{c_{\mathrm{g}}^{2}} \pm\left|\frac{T_{\mathrm{g}} s_{\mathrm{g}}^{2}}{C_{p, \mathrm{~g}}}\left(c_{\mathrm{g}}^{2}-c_{\ell}^{2}\right)-\frac{\xi_{\mathrm{g}}^{2} c_{\ell}^{2}}{c_{\mathrm{g}}^{2}}\right|}{2\left[\frac{T_{\mathrm{g}} s_{\mathrm{g}}^{2}}{C_{p, \mathrm{~g}}}+\frac{\xi_{\mathrm{g}}^{2}}{c_{\mathrm{g}}^{2}}\right]}
$$

which is equivalent to

$$
\lim _{\alpha_{\ell} \rightarrow 1} c_{\mu,+}=\max \left(\frac{c_{\mathrm{g}}}{\sqrt{1+\frac{\xi_{\mathrm{g}}^{2}}{c_{\mathrm{g}}^{2}} \frac{C_{p, \mathrm{~g}}}{T_{\mathrm{g}} s_{\mathrm{g}}^{2}}}}, c_{\ell}\right), \quad \text { and } \quad \lim _{\alpha_{\ell} \rightarrow 1} c_{\mu,-}=\min \left(\frac{c_{\mathrm{g}}}{\sqrt{1+\frac{\xi_{\mathrm{g}}^{2}}{c_{\mathrm{g}}^{2}} \frac{C_{p, \mathrm{~g}}}{T_{\mathrm{g}} s_{\mathrm{g}}^{2}}}}, c_{\ell}\right) .
$$

Clearly, only one of these approach the appropriate phasic value $c_{\ell}^{2}$. The result limits for $\alpha_{\mathrm{g}} \rightarrow 1$ are found by phase symmetry. Similarly, we find for the $T$-model, from (73), that

$$
\lim _{\alpha_{\ell} \rightarrow 1} c_{T,+}=\min \left(\frac{c_{\mathrm{g}}}{\sqrt{1+\frac{\Gamma_{\mathrm{g}}^{2} C_{p, \mathrm{~g}} T}{c_{\mathrm{g}}^{2}}}}, c_{\ell}\right), \quad \text { and } \quad \lim _{\alpha_{\ell} \rightarrow 1} c_{T,-}=\min \left(\frac{c_{\mathrm{g}}}{\sqrt{1+\frac{\Gamma_{\mathrm{g}}^{2} C_{p, \mathrm{~g}} T}{c_{\mathrm{g}}^{2}}}}, c_{\ell}\right) \text {, }
$$

to which the same observation applies.

The remaining sound speeds are continuous at the phase boundary; for the $T \mu$-model in the sense that $\lim _{\alpha_{k} \rightarrow 1} c_{T \mu,+}=c_{k}$ and $\lim _{\alpha_{k} \rightarrow 1} c_{T \mu,-}=0$, which can be deduced from the analytic expression (144).

TABle 2. Parameters for a two-phase $\mathrm{CO}_{2}$ mixture at 50 bar.

\begin{tabular}{lllcc}
\hline Quantity & Symbol & Unit & Gas & Liquid \\
\hline Pressure & $p$ & $\mathrm{MPa}$ & 5.0 & 5.0 \\
Temperature & $T$ & $\mathrm{~K}$ & 287.43 & 287.43 \\
Density & $\rho$ & $\mathrm{kg} \mathrm{m}^{-3}$ & 156.71 & 827.21 \\
Speed of sound & $c$ & $\mathrm{~m} \mathrm{~s}^{-1}$ & 201.54 & 398.89 \\
Heat capacity & $C_{p}$ & $\mathrm{~J} \mathrm{~kg}^{-1} \mathrm{~K}^{-1}$ & 3138.0 & 3356.9 \\
Entropy & $s$ & $\mathrm{~m}^{2} \mathrm{~s}^{-2} \mathrm{~K}^{-1}$ & 1753.9 & 1128.8 \\
Grüneisen coefficient & $\Gamma$ & $(-)$ & 0.30949 & 0.63175 \\
\hline
\end{tabular}

\subsection{Physical considerations}

It is commonly argued that pressure relaxation is a much faster process than the other relaxation processes $[58,71]$. Temperature relaxation, or heat flow, is associated with diffusion, which is an intrinsically slow process. Chemical potential relaxation, i.e. mass transfer, is also slow compared to pressure relaxation. Zein et al. [71] provide interesting discussions on the topic and argue that temperature relaxation is faster than chemical relaxation. Generally, the magnitudes of the different relaxation times may be strongly problem-dependent. 
Such considerations may have implications, e.g., for the mass flow through a nozzle, which has been shown to be linked to the subcharacteristic condition [39].

Apart from this, effects not captured by the coarse-grained flow models may come into play, and which model is more accurate may depend heavily on the flow regime under consideration. The effects that arise from having independent phasic pressures may be of importance for the wave dynamics of the system, and thus models with different pressures may be sensible, even though the associated relaxation time is commonly thought to be comparatively short. With regards to evaluating the physical relevance of the models presented herein, experimental data on sound speeds in two-phase flow can be found for various systems $[36,61,64,69]$.

\subsection{Numerical considerations}

A well-known problem with $p$-relaxed (one-pressure) two-fluid models is that they develop complex eigenvalues when $v_{\mathrm{g}} \neq v_{\ell}$. This is commonly resolved e.g. by adding a regularising pressure which enforces hyperbolicity $[11,14,19,51,65]$. It is worth noting that the two-fluid models with independent phasic pressures, i.e. the $T$-, $\mu$ - and $T \mu$-models, are locally hyperbolic even for small perturbations away from velocity equilibrium, due to the following argument: An eigenvalue of a matrix with real coefficients may only be complex if its complex conjugate is also an eigenvalue. Since the eigenvalues of the individual phasic pressure models are real and distinct when $\varepsilon=v_{\mathrm{g}}-v_{\ell}=0$, they must remain so for sufficiently small $\varepsilon$, as the eigenvalues may only become complex in a continuous way. In order to determine how large $\varepsilon$ may be before hyperbolicity is lost, we must find the higher-order corrections in $\varepsilon$ to the eigenvalues, which is beyond the scope of this work.

\section{Conclusions And Further WORK}

In this paper, we have presented and completed a hierarchy of relaxation models for two-phase flow, which arises when we impose instantaneous equilibrium in different combinations of velocity, pressure, temperature and chemical potential. The starting point of the analysis has been the classic seven-equation Baer-Nunziato model [4] equipped with relaxation source terms. We have in the present work provided the $T-, \mu_{-}, p \mu-$, and $T \mu$-models, which represent original contributions to the hierarchy. Explicit expressions for the sound speeds of these models have been derived. Using the new expressions and results from the literature, we have shown analytically that the subcharacteristic condition is always satisfied in the hierarchy, given velocity equilibrium between the phases.

To this end, we have contributed with 15 new subcharacteristic conditions, stated in propositions 3.1-8.4. Out of these, five have been shown in a strong sense, and ten hold in a weak sense, i.e. given equilibrium in velocity.

Due to resonance, non-conservative terms and lack of strictly convex entropy it is not clear to which extent these results could be derived from general principles. This should be addressed in further works. Also, the hierarchy could be extended to multi-component or multi-phase flow, as already initiated by [30,31, 47]. Moreover, the different models could be implemented and studied numerically for relevant cases (cf. [57]). Upon comparison with experimental data, one may then unravel under which conditions the different models are admissible.

The authors are greatly indebted to H. Lund for invaluable advice and useful comments on the manuscript. Further, the first author thanks U. J. F. Aarsnes, S. Bore, A. Bolet, A. Aasen, A. Morin, S. T. Munkejord, and M. Hammer for helpful discussions. The second author enjoyed fruitful discussions with the participants of the Workshop on Compressible Multiphase Flows, Strasbourg, France, 23-25 May, 2018.

This work has been carried out with support from the BIGCCS Centre, performed under the Norwegian research program Centres for Environment-friendly Energy Research (FME), from the 3D Multifluid Flow project at SINTEF Energy Research, and from the European Union's Horizon 2020 research and innovation program through Marie Curie initial training networks under grant agreement 642976 (NanoHeal). The following partners are acknowledged for their contributions: ConocoPhillips, Gassco, Shell, Statoil, TOTAL, GDF SUEZ and the Research Council of Norway (193816/S60). 


\section{A. Coefficients in the $p \mu$-MOdel}

The coefficients in the $p \mu$-model are given by

$$
\begin{aligned}
& \mathcal{P}_{p}^{p \mu}=\frac{\alpha_{\mathrm{g}} \alpha_{\ell} \kappa_{\mu}}{\rho_{\mathrm{g}} c_{\mathrm{g}}^{2} \rho_{\ell} c_{\ell}^{2} \kappa_{p \mu}}, \quad \mathcal{G}_{p}^{p \mu}=\frac{1}{\kappa_{p \mu}}\left[\frac{\alpha_{\mathrm{g}}}{\rho_{\mathrm{g}} c_{\mathrm{g}}^{2}}+\frac{\alpha_{\ell}}{\rho_{\ell} c_{\ell}^{2}}+\frac{\alpha_{\ell} \Gamma_{\mathrm{g}} T_{\mathrm{g}}+\alpha_{\mathrm{g}} \Gamma_{\ell} T_{\ell}}{\rho_{\mathrm{g}} c_{\mathrm{g}}^{2} \rho_{\ell} c_{\ell}^{2}} s^{*}\right], \\
& \mathcal{P}_{\mu}^{p \mu}=\frac{\alpha_{\mathrm{g}} \alpha_{\ell}}{\rho_{\mathrm{g}} c_{\mathrm{g}}^{2} \rho_{\ell} c_{\ell}^{2} \kappa_{p \mu}}\left(\frac{\xi_{\mathrm{g}}}{\alpha_{\mathrm{g}}}+\frac{\xi_{\ell}}{\alpha_{\ell}}\right), \quad \mathcal{G}_{\mu}^{p \mu}=-\frac{1}{\kappa_{p \mu}}\left(\frac{\alpha_{\mathrm{g}}}{\rho_{\mathrm{g}} c_{\mathrm{g}}^{2}}+\frac{\alpha_{\ell}}{\rho_{\ell} c_{\ell}^{2}}\right), \quad \mathcal{V}_{\mu, \mathrm{g}}^{p \mu}=-\frac{1}{\kappa_{p \mu}}\left(\frac{\xi_{\mathrm{g}}}{\rho_{\mathrm{g}} c_{\mathrm{g}}^{2}}-\frac{\xi_{\ell}}{\rho_{\ell} c_{\ell}^{2}}\right), \\
& I_{v}^{p \mu}=\frac{\alpha_{\mathrm{g}} \alpha_{\ell}}{\rho_{\mathrm{g}} c_{\mathrm{g}}^{2} \rho_{\ell} c_{\ell}^{2} \kappa_{p \mu}}\left\{\left(\frac{s_{\mathrm{g}}}{\tilde{C}_{p, \mathrm{~g}}}\left[\frac{\xi_{\mathrm{g}}}{\alpha_{\mathrm{g}}}+\frac{\xi_{\ell}}{\alpha_{\ell}}\right]+\frac{\Gamma_{\mathrm{g}}}{\alpha_{\mathrm{g}}}\left[\frac{T_{\mathrm{g}} s_{\mathrm{g}}^{2}}{\tilde{C}_{p, \mathrm{~g}}}+\frac{T_{\ell} s_{\ell}^{2}}{\tilde{C}_{p, \ell}}\right]-\frac{\Gamma_{\mathrm{g}} \xi_{\ell}}{\alpha_{\mathrm{g}} \alpha_{\ell}}\left[\frac{\xi_{\mathrm{g}}}{\rho_{\mathrm{g}} c_{\mathrm{g}}^{2}}-\frac{\xi_{\ell}}{\rho_{\ell} c_{\ell}^{2}}\right]\right.\right. \\
& \left.-\left[\frac{\Gamma_{\mathrm{g}} \Gamma_{\ell}}{\alpha_{\mathrm{g}} \alpha_{\ell}}\left(\frac{\xi_{\mathrm{g}}}{\rho_{\mathrm{g}} c_{\mathrm{g}}^{2}}-\frac{\xi_{\ell}}{\rho_{\ell} c_{\ell}^{2}}\right)+\frac{\Gamma_{\mathrm{g}} s_{\ell}}{\alpha_{\mathrm{g}} \tilde{C}_{p, \ell}}-\frac{\Gamma_{\ell} s_{\mathrm{g}}}{\alpha_{\ell} \tilde{C}_{p, \mathrm{~g}}}\right] T_{\ell} s^{*}\right) \Delta_{\mathrm{i}} v_{\mathrm{g}} \\
& +\left(\frac{s_{\ell}}{\tilde{C}_{p, \ell}}\left[\frac{\xi_{\mathrm{g}}}{\alpha_{\mathrm{g}}}+\frac{\xi_{\ell}}{\alpha_{\ell}}\right]+\frac{\Gamma_{\ell}}{\alpha_{\ell}}\left[\frac{T_{\mathrm{g}} s_{\mathrm{g}}^{2}}{\tilde{C}_{p, \mathrm{~g}}}+\frac{T_{\ell} s_{\ell}^{2}}{\tilde{C}_{p, \ell}}\right]+\frac{\Gamma_{\ell} \xi_{\mathrm{g}}}{\alpha_{\mathrm{g}} \alpha_{\ell}}\left[\frac{\xi_{\mathrm{g}}}{\rho_{\mathrm{g}} c_{\mathrm{g}}^{2}}-\frac{\xi_{\ell}}{\rho_{\ell} c_{\ell}^{2}}\right]\right. \\
& \left.\left.+\left[\frac{\Gamma_{\mathrm{g}} \Gamma_{\ell}}{\alpha_{\mathrm{g}} \alpha_{\ell}}\left(\frac{\xi_{\mathrm{g}}}{\rho_{\mathrm{g}} c_{\mathrm{g}}^{2}}-\frac{\xi_{\ell}}{\rho_{\ell} c_{\ell}^{2}}\right)+\frac{\Gamma_{\mathrm{g}} s_{\ell}}{\alpha_{\mathrm{g}} \tilde{C}_{p, \ell}}-\frac{\Gamma_{\ell} s_{\mathrm{g}}}{\alpha_{\ell} \tilde{C}_{p, \mathrm{~g}}}\right] T_{\mathrm{g}} s^{*}\right) \Delta_{\mathrm{i}} v_{\ell}\right\}, \\
& I_{T}^{p \mu}=\frac{\alpha_{\mathrm{g}} \alpha_{\ell}}{\rho_{\mathrm{g}} c_{\mathrm{g}}^{2} \rho_{\ell} c_{\ell}^{2} \kappa_{p \mu}}\left\{\left(\frac{s_{\mathrm{g}}}{\tilde{C}_{p, \mathrm{~g}}}+\frac{s_{\ell}}{\tilde{C}_{p, \ell}}\right)\left[\frac{\xi_{\mathrm{g}}}{\alpha_{\mathrm{g}}}+\frac{\xi_{\ell}}{\alpha_{\ell}}\right]+\left(\frac{\Gamma_{\mathrm{g}}}{\alpha_{\mathrm{g}}}+\frac{\Gamma_{\ell}}{\alpha_{\ell}}\right)\left[\frac{T_{\mathrm{g}} s_{\mathrm{g}}^{2}}{\tilde{C}_{p, \mathrm{~g}}}+\frac{T_{\ell} s_{\ell}^{2}}{\tilde{C}_{p, \ell}}\right]\right. \\
& \left.-\frac{\Gamma_{\mathrm{g}} \xi_{\ell}-\Gamma_{\ell} \xi_{\mathrm{g}}}{\alpha_{\mathrm{g}} \alpha_{\ell}}\left[\frac{\xi_{\mathrm{g}}}{\rho_{\mathrm{g}} c_{\mathrm{g}}^{2}}-\frac{\xi_{\ell}}{\rho_{\ell} c_{\ell}^{2}}\right]-\left[\frac{\Gamma_{\mathrm{g}} \Gamma_{\ell}}{\alpha_{\mathrm{g}} \alpha_{\ell}}\left(\frac{\xi_{\mathrm{g}}}{\rho_{\mathrm{g}} c_{\mathrm{g}}^{2}}-\frac{\xi_{\ell}}{\rho_{\ell} c_{\ell}^{2}}\right)+\frac{\Gamma_{\mathrm{g}} s_{\ell}}{\alpha_{\mathrm{g}} \tilde{C}_{p, \ell}}-\frac{\Gamma_{\ell} s_{\mathrm{g}}}{\alpha_{\ell} \tilde{C}_{p, \mathrm{~g}}}\right]\left(T_{\ell}-T_{\mathrm{g}}\right) s^{*}\right\}, \\
& \mathcal{K}_{v}^{p \mu}=\frac{1}{\kappa_{p \mu}}\left\{\left[\left(\frac{\alpha_{\mathrm{g}}}{\rho_{\mathrm{g}} c_{\mathrm{g}}^{2}}+\frac{\alpha_{\ell}}{\rho_{\ell} c_{\ell}^{2}}\right) \frac{s_{\mathrm{g}}}{\tilde{C}_{p, \mathrm{~g}}}-\frac{\Gamma_{\mathrm{g}}}{\rho_{\mathrm{g}} c_{\mathrm{g}}^{2}}\left(\frac{\xi_{\mathrm{g}}}{\rho_{\mathrm{g}} c_{\mathrm{g}}^{2}}-\frac{\xi_{\ell}}{\rho_{\ell} c_{\ell}^{2}}\right)\right] \Delta_{\mathrm{i}} v_{\mathrm{g}}+\left[\left(\frac{\alpha_{\mathrm{g}}}{\rho_{\mathrm{g}} c_{\mathrm{g}}^{2}}+\frac{\alpha_{\ell}}{\rho_{\ell} c_{\ell}^{2}}\right) \frac{s_{\ell}}{\tilde{C}_{p, \ell}}+\frac{\Gamma_{\ell}}{\rho_{\ell} c_{\ell}^{2}}\left(\frac{\xi_{\mathrm{g}}}{\rho_{\mathrm{g}} c_{\mathrm{g}}^{2}}-\frac{\xi_{\ell}}{\rho_{\ell} c_{\ell}^{2}}\right)\right] \Delta_{\mathrm{i}} v_{\ell}\right\}, \\
& \mathcal{K}_{T}^{p \mu}=\frac{1}{\kappa_{p \mu}}\left\{\left(\frac{\alpha_{\mathrm{g}}}{\rho_{\mathrm{g}} c_{\mathrm{g}}^{2}}+\frac{\alpha_{\ell}}{\rho_{\ell} c_{\ell}^{2}}\right)\left(\frac{s_{\mathrm{g}}}{\tilde{C}_{p, \mathrm{~g}}}+\frac{s_{\ell}}{\tilde{C}_{p, \ell}}\right)-\left(\frac{\Gamma_{\mathrm{g}}}{\rho_{\mathrm{g}} c_{\mathrm{g}}^{2}}-\frac{\Gamma_{\ell}}{\rho_{\ell} c_{\ell}^{2}}\right)\left(\frac{\xi_{\mathrm{g}}}{\rho_{\mathrm{g}} c_{\mathrm{g}}^{2}}-\frac{\xi_{\ell}}{\rho_{\ell} c_{\ell}^{2}}\right)\right\} \\
& \Phi_{\mathrm{g}}=\frac{1}{\kappa_{p \mu}}\left\{\frac{\alpha_{\ell}}{\rho_{\ell} c_{\ell}^{2}}\left(\frac{T_{\mathrm{g}} s_{\mathrm{g}}^{2}}{\tilde{C}_{p, \mathrm{~g}}}+\frac{T_{\ell} s_{\ell}^{2}}{\tilde{C}_{p, \ell}}\right)-\frac{\xi_{\ell}}{\rho_{\ell} c_{\ell}^{2}}\left(\frac{\xi_{\mathrm{g}}}{\rho_{\mathrm{g}} c_{\mathrm{g}}^{2}}-\frac{\xi_{\ell}}{\rho_{\ell} c_{\ell}^{2}}\right)-\left[\frac{\alpha_{\ell}}{\rho_{\ell} c_{\ell}^{2}}\left(\frac{T_{\mathrm{g}} s_{\mathrm{g}}}{\tilde{C}_{p, \mathrm{~g}}}+\frac{T_{\ell} s_{\ell}}{\tilde{C}_{p, \ell}}\right)+\left(\frac{\xi_{\mathrm{g}}}{\rho_{\mathrm{g}} c_{\mathrm{g}}^{2}}-\frac{\xi_{\ell}}{\rho_{\ell} c_{\ell}^{2}}\right) \frac{\Gamma_{\ell} T_{\ell}}{\rho_{\ell} c_{\ell}^{2}}\right] s^{*}\right\} .
\end{aligned}
$$

Herein, we have defined the shorthand denominator

$$
\begin{aligned}
\kappa_{p \mu}=\left(\frac{\alpha_{\mathrm{g}}}{\rho_{\mathrm{g}} c_{\mathrm{g}}^{2}}+\frac{\alpha_{\ell}}{\rho_{\ell} c_{\ell}^{2}}\right)\left(\frac{T_{\mathrm{g}} s_{\mathrm{g}}^{2}}{\tilde{C}_{p, \mathrm{~g}}}+\frac{T_{\ell} s_{\ell}^{2}}{\tilde{C}_{p, \ell}}\right)+\left(\frac{\xi_{\mathrm{g}}}{\rho_{\mathrm{g}} c_{\mathrm{g}}^{2}}-\frac{\xi_{\ell}}{\rho_{\ell} c_{\ell}^{2}}\right)^{2} \\
-\left[\left(\frac{\alpha_{\mathrm{g}}}{\rho_{\mathrm{g}} c_{\mathrm{g}}^{2}}+\frac{\alpha_{\ell}}{\rho_{\ell} c_{\ell}^{2}}\right)\left(\frac{T_{\mathrm{g}} s_{\mathrm{g}}}{\tilde{C}_{p, \mathrm{~g}}}+\frac{T_{\ell} s_{\ell}}{\tilde{C}_{p, \ell}}\right)-\left(\frac{\xi_{\mathrm{g}}}{\rho_{\mathrm{g}} c_{\mathrm{g}}^{2}}-\frac{\xi_{\ell}}{\rho_{\ell} c_{\ell}^{2}}\right)\left(\frac{\Gamma_{\mathrm{g}} T_{\mathrm{g}}}{\rho_{\mathrm{g}} c_{\mathrm{g}}^{2}}-\frac{\Gamma_{\ell} T_{\ell}}{\rho_{\ell} c_{\ell}^{2}}\right)\right] s^{*}
\end{aligned}
$$

and an entropy contribution due to velocity differences

$$
s^{*}=\left(v_{\mathrm{g}}-v_{\ell}\right)^{2} /\left[2\left(\sqrt{T_{\mathrm{g}}}+\sqrt{T_{\ell}}\right)^{2}\right] .
$$

The coefficients related to the quasi-linear form are given by

$$
\begin{gathered}
P_{\bar{v}}^{p \mu}=q_{p}^{p \mu}-\left(\frac{\beta_{\ell}}{\rho_{\mathrm{g}}}+\frac{\beta_{\mathrm{g}}}{\rho_{\ell}}\right) \mathcal{P}_{\mu}^{p \mu}, \quad G_{\bar{v}}^{p \mu}=\mathcal{G}_{p}^{p \mu}-\left(\frac{\beta_{\ell}}{\rho_{\mathrm{g}}}+\frac{\beta_{\mathrm{g}}}{\rho_{\ell}}\right) G_{\mu}^{p \mu}, \quad V_{\bar{v}, \mathrm{~g}}^{p \mu}=\left(\frac{\beta_{\ell}}{\rho_{\mathrm{g}}}+\frac{\beta_{\mathrm{g}}}{\rho_{\ell}}\right) \mathcal{V}_{\mu, \mathrm{g}}^{p \mu}, \\
\Psi_{\mathrm{g}}^{p}=\frac{1}{\kappa_{p \mu}}\left[\frac{\alpha_{\mathrm{g}}}{\rho_{\mathrm{g}} c_{\mathrm{g}}^{2}}\left(\frac{T_{\mathrm{g}} s_{\mathrm{g}}\left(s_{\mathrm{g}}-s^{*}\right)}{\tilde{C}_{p, \mathrm{~g}}}+\frac{T_{\ell} s_{\ell}\left(s_{\ell}-s^{*}\right)}{\tilde{C}_{p, \ell}}\right)+\frac{\xi_{\mathrm{g}}}{\rho_{\mathrm{g}} c_{\mathrm{g}}^{2}}\left(\frac{\xi_{\mathrm{g}}+\Gamma_{\mathrm{g}} T_{\mathrm{g}} s^{*}}{\rho_{\mathrm{g}} c_{\mathrm{g}}^{2}}-\frac{\xi_{\ell}+\Gamma_{\ell} T_{\ell} s^{*}}{\rho_{\ell} c_{\ell}^{2}}\right)\right], \\
G_{p}^{p \mu}=\frac{1}{\kappa_{p \mu}}\left[\frac{\xi_{\mathrm{g}}+\Gamma_{\mathrm{g}} T_{\mathrm{g}} s^{*}}{\rho_{\mathrm{g}} c_{\mathrm{g}}^{2}}-\frac{\xi_{\ell}+\Gamma_{\ell} T_{\ell} s^{*}}{\rho_{\ell} c_{\ell}^{2}}\right], \quad V_{p}^{p \mu}=\frac{1}{\kappa_{p \mu}}\left[\frac{T_{\mathrm{g}} s_{\mathrm{g}}^{2}}{\tilde{C}_{p, \mathrm{~g}}}+\frac{T_{\ell} s_{\ell}^{2}}{\tilde{C}_{p, \ell}}-\left(\frac{T_{\mathrm{g}} s_{\mathrm{g}}}{\tilde{C}_{p, g}}+\frac{T_{\ell} s_{\ell}}{\tilde{C}_{p, \ell}}\right) s^{*}\right], \\
P_{\mu}^{p \mu}=\frac{\xi_{\mathrm{g}} \xi_{\ell}}{\rho_{\mathrm{g}} c_{\mathrm{g}}^{2} \rho_{\ell} c_{\ell}^{2} \kappa_{p \mu}}\left\{\frac{\xi_{\mathrm{g}}}{\rho_{\mathrm{g}} c_{\mathrm{g}}^{2}}-\frac{\xi_{\ell}}{\rho_{\ell} c_{\ell}^{2}}+\frac{\alpha_{\mathrm{g}} T_{\mathrm{g}} s_{\mathrm{g}}^{2}}{\xi_{\mathrm{g}} \tilde{C}_{p, \mathrm{~g}}}-\frac{\alpha_{\ell} T_{\ell} s_{\ell}^{2}}{\xi_{\ell} \tilde{C}_{p, \ell}}-\left[\frac{\Gamma_{\ell} T_{\ell}}{\rho_{\ell} c_{\ell}^{2}}-\frac{\alpha_{\ell} T_{\ell} s_{\ell}}{\xi_{\ell} \tilde{C}_{p, \ell}}-\frac{\Gamma_{\mathrm{g}} T_{\mathrm{g}}}{\rho_{\mathrm{g}} c_{\mathrm{g}}^{2}}+\frac{\alpha_{\mathrm{g}} T_{\mathrm{g}} s_{\mathrm{g}}}{\xi_{\mathrm{g}} \tilde{C}_{p, \mathrm{~g}}}\right] s^{*}\right\},
\end{gathered}
$$




$$
\begin{gathered}
\Psi_{\mathrm{g}}^{\mu}=\frac{1}{\kappa_{p \mu}}\left[\left(\frac{\alpha_{\mathrm{g}}}{\rho_{\mathrm{g}} c_{\mathrm{g}}^{2}}+\frac{\alpha_{\ell}}{\rho_{\ell} c_{\ell}^{2}}\right) \frac{T_{\mathrm{g}} s_{\mathrm{g}}\left(s_{\mathrm{g}}-s^{*}\right)}{\tilde{C}_{p, \mathrm{~g}}}-\frac{\xi_{\ell}}{\rho_{\ell} c_{\ell}^{2}}\left(\frac{\xi_{\mathrm{g}}+\Gamma_{\mathrm{g}} T_{\mathrm{g}} s^{*}}{\rho_{\mathrm{g}} c_{\mathrm{g}}^{2}}-\frac{\xi_{\ell}+\Gamma_{\ell} T_{\ell} s^{*}}{\rho_{\ell} c_{\ell}^{2}}\right)\right], \\
V_{\mu}^{p \mu}=\frac{1}{\kappa_{p \mu}}\left[\frac{\xi_{\ell}}{\rho_{\ell} c_{\ell}^{2}} \frac{T_{\mathrm{g}} s_{\mathrm{g}}\left(s_{\mathrm{g}}-s^{*}\right)}{\tilde{C}_{p, \mathrm{~g}}}+\frac{\xi_{\mathrm{g}}}{\rho_{\mathrm{g}} c_{\mathrm{g}}^{2}} \frac{T_{\ell} s_{\ell}\left(s_{\ell}-s^{*}\right)}{\tilde{C}_{p, \ell}}\right] .
\end{gathered}
$$

\section{B. COefficients in The $T \mu$-MOdeL}

The coefficients in the $T \mu$-model are given by

$$
\begin{aligned}
& \mathcal{A}_{\mu}^{T \mu}=\frac{\left(p_{\mathrm{g}}-p_{\ell}\right)\left(v_{\mathrm{g}}+v_{\ell}\right)}{2} A_{\mathrm{g}}^{T \mu}, \quad \mathcal{G}_{\mu}^{T \mu}=-\frac{m_{\mathrm{g}} m_{\ell}}{c_{\mathrm{g}}^{2} c_{\ell}^{2} T \kappa_{T \mu}}\left[\frac{\zeta_{\mathrm{g}}}{\tilde{C}_{p, \mathrm{~g}}}+\frac{\zeta_{\ell}}{\tilde{C}_{p, \ell}}\right], \\
& \mathcal{T}_{\mu}^{T \mu}=\frac{m_{\mathrm{g}} m_{\ell}}{c_{\mathrm{g}}^{2} c_{\ell}^{2} T \kappa_{T \mu}}\left[\frac{\Gamma_{\mathrm{g}}}{m_{\mathrm{g}}}+\frac{\Gamma_{\ell}}{m_{\ell}}-\frac{s_{\mathrm{g}} \zeta_{\mathrm{g}}}{\tilde{C}_{p, \mathrm{~g}}}-\frac{s_{\ell} \zeta_{\ell}}{\tilde{C}_{p, \ell}}\right] \text {, } \\
& A_{\mathrm{g}}^{T \mu}=\frac{m_{\mathrm{g}} m_{\ell}}{c_{\mathrm{g}}^{2} c_{\ell}^{2} T \kappa_{T \mu}}\left[\frac{\Gamma_{\ell} \zeta_{\mathrm{g}}}{m_{\ell} \tilde{C}_{p, \mathrm{~g}}}-\frac{\Gamma_{\mathrm{g}} \zeta_{\ell}}{m_{\mathrm{g}} \tilde{C}_{p, \ell}}+\frac{\zeta_{\mathrm{g}} \zeta_{\ell} \Delta h}{\tilde{C}_{p, \mathrm{~g}} \tilde{C}_{p, \ell} T}\right], \\
& \mathcal{V}_{\mu, \mathrm{g}}^{T \mu}=\frac{m_{\mathrm{g}} m_{\ell}}{c_{\mathrm{g}}^{2} c_{\ell}^{2} T \kappa_{T \mu}}\left[-\frac{c_{\mathrm{g}}^{2}}{\alpha_{\mathrm{g}}}\left(\frac{1}{\tilde{C}_{p, \mathrm{~g}}}+\frac{1}{\tilde{C}_{p, \ell}}\right)+\frac{c_{\mathrm{g}}^{2} c_{\ell}^{2}}{\alpha_{\mathrm{g}} \alpha_{\ell}} \frac{\Gamma_{\ell} T}{\rho_{\ell} c_{\ell}^{2}}\left(\frac{\Gamma_{\mathrm{g}}}{c_{\mathrm{g}}^{2}}-\frac{\Gamma_{\ell}}{c_{\ell}^{2}}\right)+\frac{\Gamma_{\mathrm{g}} \Delta h}{\alpha_{\mathrm{g}}}\left(\frac{1}{\tilde{C}_{p, \ell}}+\frac{\Gamma_{\ell}^{2} T}{m_{\ell} c_{\ell}^{2}}\right)\right], \\
& \mathcal{V}_{\mu, \ell}^{T \mu}=\frac{m_{\mathrm{g}} m_{\ell}}{c_{\mathrm{g}}^{2} c_{\ell}^{2} T \kappa_{T \mu}}\left[-\frac{c_{\ell}^{2}}{\alpha_{\ell}}\left(\frac{1}{\tilde{C}_{p, \mathrm{~g}}}+\frac{1}{\tilde{C}_{p, \ell}}\right)-\frac{c_{\mathrm{g}}^{2} c_{\ell}^{2}}{\alpha_{\mathrm{g}} \alpha_{\ell}} \frac{\Gamma_{\mathrm{g}} T}{\rho_{\mathrm{g}} c_{\mathrm{g}}^{2}}\left(\frac{\Gamma_{\mathrm{g}}}{c_{\mathrm{g}}^{2}}-\frac{\Gamma_{\ell}}{c_{\ell}^{2}}\right)-\frac{\Gamma_{\ell} \Delta h}{\alpha_{\ell}}\left(\frac{1}{\tilde{C}_{p, \mathrm{~g}}}+\frac{\Gamma_{\mathrm{g}}^{2} T}{m_{\mathrm{g}} c_{\mathrm{g}}^{2}}\right)\right], \\
& \mathcal{K}_{p}^{T \mu}=-\left(p_{\mathrm{g}}-p_{\ell}\right) A_{\mathrm{g}}^{T \mu}+\mathcal{V}_{\mu, \mathrm{g}}^{T \mu}+\mathcal{V}_{\mu, \ell}^{T \mu}, \quad \mathcal{K}_{\mathrm{v}}^{T \mu}=-A_{\mathrm{g}}^{T \mu}, \\
& \kappa_{T \mu}=\frac{\Delta h^{2} m_{\mathrm{g}} m_{\ell}}{\tilde{C}_{p, \mathrm{~g}} \tilde{C}_{p, \ell} T^{2} c_{\mathrm{g}}^{2} c_{\ell}^{2}}+\frac{\frac{m_{\ell}}{c_{\ell}^{2}}+\frac{m_{\mathrm{g}}}{c_{\mathrm{g}}^{2}}\left(1+\frac{\Gamma_{\ell}}{c_{\ell}^{2}} \Delta h\right)^{2}}{\tilde{C}_{p, \mathrm{~g}} T}+\frac{\frac{m_{\mathrm{g}}}{c_{\mathrm{g}}^{2}}+\frac{m_{\ell}}{c_{\ell}^{2}}\left(1-\frac{\Gamma_{\mathrm{g}}}{c_{\mathrm{g}}^{2}} \Delta h\right)^{2}}{\tilde{C}_{p, \ell} T}+\left(\frac{\Gamma_{\ell}}{c_{\ell}^{2}}-\frac{\Gamma_{\mathrm{g}}}{c_{\mathrm{g}}^{2}}-\frac{\Gamma_{\mathrm{g}} \Gamma_{\ell}}{c_{\mathrm{g}}^{2} c_{\ell}^{2}} \Delta h\right)^{2} .
\end{aligned}
$$

\section{REFERENCES}

[1] Ulf Jakob F Aarsnes, Tore Flåtten, and Ole Morten Aamo. Review of two-phase flow models for control and estimation. Annu. Rev. Control, 42:50-62, 2016.

[2] Grégoire Allaire, Sébastien Clerc, and Samuel Kokh. A five-equation model for the simulation of interfaces between compressible fluids. J. Comput. Phys., 181(2):577-616, 2002.

[3] M. Bachmann, S. Müller, P. Helluy, and H Mathis. A simple model for cavitation with non-condensable gases. Series in Contemporary Applied Mathematics: Hyperbolic Problems, pages 289-296, 2012.

[4] M. R. Baer and J. W. Nunziato. A two-phase mixture theory for the deflagration-to-detonation transition (ddt) in reactive granular materials. Int. J. Multiphase Flow, 12(6):861-889, 1986.

[5] Thomas Barberon and Philippe Helluy. Finite volume simulation of cavitating flows. Comput. Fluids, 34(7):832-858, 2005.

[6] Michaël Baudin, Christophe Berthon, Frédéric Coquel, Roland Masson, and Quang Huy Tran. A relaxation method for twophase flow models with hydrodynamic closure law. Numer. Math., 99(3):411-440, 2005.

[7] Michaël Baudin, Frédéric Coquel, and Quang-Huy Tran. A semi-implicit relaxation scheme for modeling two-phase flow in a pipeline. SIAM J. Sci. Comput., 27(3):914-936, 2005.

[8] J. B. Bdzil, R. Menikoff, S. F. Son, A. K. Kapila, and D. Scott Stewart. Two-phase modeling of deflagration-to-detonation transition in granular materials: A critical examination of modeling issues. Phys. Fluids, 11(2):378-402, 1999.

[9] Kjell H Bendiksen, Dag Malnes, Randi Moe, Sven Nuland, et al. The dynamic two-fluid model OLGA: Theory and application. SPE Prod. Eng., 6(02):171-180, 1991.

[10] Torodd Berstad, Cato Dørum, Jana Poplsteinova Jakobsen, Steinar Kragset, Hailong Li, Halvor Lund, Alexandre Morin, Svend Tollak Munkejord, Mona Jacobsen Mølnvik, Håkon Ottar Nordhagen, et al. CO2 pipeline integrity: A new evaluation methodology. Energy Procedia, 4:3000-3007, 2011.

[11] Dominique Bestion. The physical closure laws in the CATHARE code. Nuclear Engineering and Design, 124(3):229-245, 1990.

[12] Gui-Qiang Chen, C. David Levermore, and Tai-Ping Liu. Hyperbolic conservation laws with stiff relaxation terms and entropy. Comm. Pure Appl. Math., 47(6):787-830, 1994.

[13] S. Clerc. Numerical simulation of the homogeneous equilibrium model for two-phase flows. J. Comput. Phys., 161(1):354-375, 2000.

[14] F. Coquel, K. El Amine, E. Godlewski, B. Perthame, and P. Rascle. A numerical method using upwind schemes for the resolution of two-phase flows. J. Comput. Phys., 136(2):272-288, 1997. 
[15] Frédéric Coquel, Thierry Gallouët, Jean-Marc Hérard, and Nicolas Seguin. Closure laws for a two-fluid two-pressure model. C. R. Acad. Sci. Paris, 334(10):927-932, 2002.

[16] Frédéric Coquel, Jean-Marc Hérard, Khaled Saleh, and Nicolas Seguin. Two properties of two-velocity two-pressure models for two-phase flows. Commun. Math. Sci., 12(3):593-600, 2014.

[17] J. Cortes, A. Debussche, and I. Toumi. A density perturbation method to study the eigenstructure of two-phase flow equation systems. J. Comput. Phys., 147(2):463-484, 1998.

[18] Donald A Drew and Stephen L Passman. Theory of multicomponent fluids, volume 135. Springer-Verlag, 1999.

[19] Steinar Evje and Tore Flåtten. Hybrid flux-splitting schemes for a common two-fluid model. J. Comput. Phys., 192(1):175-210, 2003.

[20] Gloria Faccanoni, Samuel Kokh, and Grégoire Allaire. Modelling and simulation of liquid-vapor phase transition in compressible flows based on thermodynamical equilibrium. ESAIM: Math. Model. Numer. Anal., 46(5):1029-1054, 2012.

[21] Pedro José Martínez Ferrer, Tore Flåtten, and Svend Tollak Munkejord. On the effect of temperature and velocity relaxation in two-phase flow models. ESAIM: Math. Model. Numer. Anal., 46(2):411-442, 2012.

[22] Tore Flåtten and Halvor Lund. Relaxation two-phase flow models and the subcharacteristic condition. Math. Models Methods Appl. Sci., 21(12):2379-2407, 2011.

[23] Tore Flåtten, Alexandre Morin, and Svend Tollak Munkejord. Wave propagation in multicomponent flow models. SIAM J. Appl. Math., 70(8):2861-2882, 2010.

[24] Thierry Gallouët, Philippe Helluy, Jean-Marc Hérard, and Julien Nussbaum. Hyperbolic relaxation models for granular flows. ESAIM: Math. Model. Numer. Anal., 44(2):371-400, 2010.

[25] Thierry Gallouët, Jean-Marc Hérard, and Nicolas Seguin. Numerical modeling of two-phase flows using the two-fluid twopressure approach. Math. Models Methods Appl. Sci., 14(05):663-700, 2004.

[26] Sergey Gavrilyuk and Henri Gouin. A new form of governing equations of fluids arising from Hamilton's principle. International Journal of Engineering Science, 37(12):1495-1520, 1999.

[27] Sergey Gavrilyuk and Richard Saurel. Mathematical and numerical modeling of two-phase compressible flows with microinertia. J. Comput. Phys., 175(1):326-360, 2002.

[28] L. G. Gvozdeva and O. A. Predvoditeleva. Triple configurations of detonation waves in gases. Combustion, Explosion and Shock Waves, 5(4):309-316, 1969.

[29] Morten Hammer and Alexandre Morin. A method for simulating two-phase pipe flow with real equations of state. Comput. Fluids, 100:45-58, 2014.

[30] E. Han, M. Hantke, and S. Müller. Efficient and robust relaxation procedures for multi-component mixtures including phase transition. J. Comput. Phys., 338:217-239, 2017.

[31] M. Hantke and S. Müller. Analysis and simulation of a new multi-component two-phase flow model with phase transitions and chemical reactions. Q. Appl. Math., 76:253-287, 2018.

[32] Philippe Helluy and Hélène Mathis. Pressure laws and fast legendre transform. Math. Models Methods Appl. Sci., 21(04):745$775,2011$.

[33] Philippe Helluy and Nicolas Seguin. Relaxation models of phase transition flows. ESAIM: Math. Model. Numer. Anal., 40(2):331-352, 2006.

[34] Mamoru Ishii and Takashi Hibiki. Thermo-fluid dynamics of two-phase flow. Springer-Verlag, 2011.

[35] A. K. Kapila, R. Menikoff, J. B. Bdzil, S. F. Son, and D. Scott Stewart. Two-phase modeling of deflagration-to-detonation transition in granular materials: Reduced equations. Phys. Fluids, 13(10):3002-3024, 2001.

[36] Susan Werner Kieffer. Sound speed in liquid-gas mixtures: Water-air and water-steam. J. Geophys. Res., 82(20):2895-2904, 1977.

[37] Mathieu Labois. Modélisation des déséquilibres mécaniques dans les écoulements diphasiques: Approches par relaxation et par modèle réduit. $\mathrm{PhD}$ thesis, Université de Provence (Aix-Marseille I), 2008.

[38] Jean Leray. Hyperbolic differential equations. The Institute for Advanced Study, 1953.

[39] Gaute Linga, Peder Aursand, and Tore Flåtten. Two-phase nozzle flow and the subcharacteristic condition. J. Math. Anal. Appl., 426(2):917-934, 2015.

[40] Gaute Linga and Halvor Lund. A two-fluid model for vertical flow applied to co2 injection wells. Int. J. Greenh. Gas Con., 51:71-80, 2016.

[41] Tai-Ping Liu. Hyperbolic conservation laws with relaxation. Commun. Math. Phys., 108(1):153-175, 1987.

[42] Halvor Lund. A hierarchy of relaxation models for two-phase flow. SIAM J. Appl. Math., 72(6):1713-1741, 2012.

[43] Halvor Lund. Relaxation models for two-phase flow with applications to $\mathrm{CO}_{2}$ transport. PhD thesis, Norges teknisknaturvitenskapelige universitet, Fakultet for ingeniørvitenskap og teknologi, Institutt for energi-og prosessteknikk, 2013.

[44] Ralph Menikoff and Bradley J. Plohr. The Riemann problem for fluid flow of real materials. Reviews of Modern Physics, 61(1):75, 1989.

[45] Alexandre Morin. Mathematical modelling and numerical simulation of two-phase multi-component flows of $\mathrm{CO}_{2}$ mixtures in pipes. PhD thesis, Norges teknisk-naturvitenskapelige universitet, Fakultet for ingeniørvitenskap og teknologi, Institutt for energi-og prosessteknikk, 2012. 
[46] Alexandre Morin and Tore Flåtten. A two-fluid four-equation model with instantaneous thermodynamical equilibrium. ESAIM: Math. Model. Numer. Anal., 50(4):1167-1192, 2016.

[47] Siegfried Müller, Maren Hantke, and Pascal Richter. Closure conditions for non-equilibrium multi-component models. Continuum Mechanics and Thermodynamics, 28(4):1157-1189, 2016.

[48] Svend Tollak Munkejord, Morten Hammer, and Sigurd W Løvseth. CO2 transport: Data and models-a review. Appl. Energy, 169:499-523, 2016.

[49] Angelo Murrone and Hervé Guillard. A five equation reduced model for compressible two phase flow problems. J. Comput. Phys., 202(2):664-698, 2005.

[50] Roberto Natalini. Recent mathematical results on hyperbolic relaxation problems. In Chapman 83 Hall/CRC Monogr. Surv. Pure Appl. Math., volume 99. Chapman \& Hall/CRC, 1999.

[51] Michaël Ndjinga. Influence of interfacial pressure on the hyperbolicity of the two-fluid model. C. R. Acad. Sci. Paris, 344(6):407-412, 2007.

[52] Marica Pelanti and Keh-Ming Shyue. A mixture-energy-consistent six-equation two-phase numerical model for fluids with interfaces, cavitation and evaporation waves. J. Comput. Phys., 259:331-357, 2014.

[53] J. Pettersen, A. Hafner, G. Skaugen, and H. Rekstad. Development of compact heat exchangers for $\mathrm{CO}_{2}$ air-conditioning systems. Int. J. Refrig., 21(3):180-193, 1998.

[54] D. J. Picard and P. R. Bishnoi. Calculation of the thermodynamic sound velocity in two-phase multicomponent fluids. Int. J. Multiphase Flow, 13(3):295-308, 1987.

[55] Philip L. Roe. Approximate riemann solvers, parameter vectors, and difference schemes. J. Comput. Phys., 43(2):357-372, 1981.

[56] Richard Saurel and Rémi Abgrall. A multiphase Godunov method for compressible multifluid and multiphase flows. J. Comput. Phys., 150(2):425-467, 1999.

[57] Richard Saurel and Carlos Pantano. Diffuse-interface capturing methods for compressible two-phase flows. Annual Review of Fluid Mechanics, 50(1), 2018.

[58] Richard Saurel, Fabien Petitpas, and Rémi Abgrall. Modelling phase transition in metastable liquids: application to cavitating and flashing flows. J. Fluid Mech., 607:313-350, 2008.

[59] Richard Saurel, Fabien Petitpas, and Ray A. Berry. Simple and efficient relaxation methods for interfaces separating compressible fluids, cavitating flows and shocks in multiphase mixtures. J. Comput. Phys., 228(5):1678-1712, 2009.

[60] Susanne Solem, Peder Aursand, and Tore Flåtten. Wave dynamics of linear hyperbolic relaxation systems. J. Hyperbol. Differ. Eq., 12(04):655-670, 2015.

[61] Herbert Städtke. Gasdynamic aspects of two-phase flow: Hyperbolicity, wave propagation phenomena and related numerical methods. John Wiley \& Sons, 2006.

[62] H. Bruce Stewart and Burton Wendroff. Two-phase flow: models and methods. J. Comput. Phys., 56(3):363-409, 1984.

[63] J. H. Stuhmiller. The influence of interfacial pressure forces on the character of two-phase flow model equations. Int. J. Multiphase Flow, 3(6):551-560, 1977.

[64] S. Tosse, Knut Vågsæther, and Dag Bjerketvedt. An experimental investigation of rapid boiling of $\mathrm{CO}_{2}$. Shock Waves, 25(3):277-282, 2015.

[65] Imad Toumi and A. Kumbaro. An approximate linearized riemann solver for a two-fluid model. J. Comput. Phys., 124(2):286300, 1996.

[66] Imad Toumi, Anela Kumbaro, and Henri Paillere. Approximate Riemann solvers and flux vector splitting schemes for two-phase flow. CEA Saclay, Direction de l'information scientifique et technique, 1999.

[67] Alexander Voss and Wolfgang Dahmen. Exact Riemann solution for the Euler equations with nonconvex and nonsmooth equation of state. PhD thesis, Fakultät für Mathematik, Informatik und Naturwissenschaften, 2005.

[68] Gerald Beresford Whitham. Linear and nonlinear waves, volume 42. John Wiley \& Sons, 1974.

[69] Jinliang Xu and Tingkuan Chen. Acoustic wave prediction in flowing steam-water two-phase mixture. Int. J. Heat Mass Tran., 43(7):1079-1088, 2000.

[70] Wen-An Yong. Basic aspects of hyperbolic relaxation systems. In Advances in the Theory of Shock Waves, pages 259-305. Springer, 2001.

[71] Ali Zein, Maren Hantke, and Gerald Warnecke. Modeling phase transition for compressible two-phase flows applied to metastable liquids. J. Comput. Phys., 229(8):2964-2998, 2010. 